University of Tennessee Health Science Center UTHSC Digital Commons

\title{
The Molecular Basis of Fitness and Transmissibility of Neuraminidase Inhibitor Resistant Influenza A Viruses
}

Susu Duan

University of Tennessee Health Science Center

Follow this and additional works at: https://dc.uthsc.edu/dissertations

Part of the Enzymes and Coenzymes Commons, Medical Immunology Commons, Medical Microbiology Commons, Medical Molecular Biology Commons, and the Virus Diseases Commons

\section{Recommended Citation}

Duan, Susu, "The Molecular Basis of Fitness and Transmissibility of Neuraminidase Inhibitor Resistant Influenza A Viruses" (2012). Theses and Dissertations (ETD). Paper 62. http://dx.doi.org/10.21007/ etd.cghs.2012.0074.

This Dissertation is brought to you for free and open access by the College of Graduate Health Sciences at UTHSC Digital Commons. It has been accepted for inclusion in Theses and Dissertations (ETD) by an authorized administrator of UTHSC Digital Commons. For more information, please contact jwelch30@uthsc.edu. 


\title{
The Molecular Basis of Fitness and Transmissibility of Neuraminidase Inhibitor Resistant Influenza A Viruses
}

\begin{abstract}
Neuraminidase (NA) inhibitors including oral oseltamivir and inhaled zanamivir are among the first line of defense against influenza virus infection. Development of resistance to NA inhibitors is a huge drawback for limited options for the control of influenza. During the first decade of NA inhibitor use, the detection rates of resistance to both NA inhibitors had remained low in circulating influenza viruses. However, the 2008 2009 season was marked by a radical increase of prevalence of oseltamvir resistance from $<1 \%$ to $>90 \%$ in worldwide surveillance in less than a year. The resistance was solely linked to NA H275Y variants of seasonal $\mathrm{H} 1 \mathrm{~N} 1$ viruses, and they are referred as the naturally resistant viruses. A big question remains open about what fundamental molecular changes in the seasonal $\mathrm{H} 1 \mathrm{~N} 1$ viruses led to the surge of the naturally resistant viruses. When this question remained pending, a novel swine-origin H1N1 influenza virus emerged in Mexico at April 2009, soon spread worldwide replacing the seasonal influenza viruses including the naturally resistant viruses, and marked 2009 with the first influenza pandemic of the $21 \mathrm{st}$ century. With sustainably increased worldwide use of NA inhibitors especially oral oseltamivir during the pandemic, the oseltamivir-resistant variants carrying H275Y NA mutation were isolated at low incidence from individuals receiving oseltamivir treatment and a few community clusters. In view of the high prevalence of naturally resistant seasonal $\mathrm{H} 1 \mathrm{~N} 1$ viruses in the immediate preceding season, there was an urgent need to characterize the transmissibility and fitness of oseltamivir-resistant pandemic H1N1/2009 viruses, although the resistance rates have remained low so far.

We first addressed the urgent question about pandemic viruses by investigating the transmissibility of a closely matched pair of pandemic $\mathrm{H} 1 \mathrm{~N} 1 / 2009$ clinical isolates, which only differed at the H275Y NA mutation in their genome, in the ferret model. We found that the H275Y NA mutant H1N1/2009 virus was not transmitted efficiently in ferrets via respiratory droplets, while it retained efficient transmission via direct contact. The wild-type H1N1/2009 virus was efficiently transmitted via both routes. The wild-type and the mutant viruses appeared to cause a similar disease course in ferrets without apparent attenuation of clinical signs. In the growth competition in a ferret, the H275Y mutant virus showed less growth capability than the wild-type virus. The NA of the H275Y mutant virus showed reduced substratebinding affinity and catalytic activity in vitro and delayed initial growth in MDCK and MDCK-SIAT1 cells. These findings may in part explain its less efficient transmission. The fact that the oseltamivir-resistant H1N1/2009 virus retained efficient transmission through direct contact underlines the necessity of continuous monitoring of drug resistance and characterization of more NA inhibitor-resistant variants of the pandemic H1N1 viruses.
\end{abstract}

We also sought to resolve the pending question about the naturally resistant seasonal H1N1 viruses by investigating the changes of different seasonal $\mathrm{H} 1 \mathrm{~N} 1$ viruses in terms of NA genetics, NA proteins attributes and virus fitness. We found that during the seasonal H1N1 virus evolution, two genetically diverged lineages of $\mathrm{H} 1 \mathrm{~N} 1$ viruses were circulating at different times. The NA protein phenotypes of the two lineages were naturally distinct in the levels of protein expression and enzyme affinity, and accordingly, the $\mathrm{H} 275$ Y NA mutation had differential effects on the NA proteins and virus fitness of the two lineages. The new lineage NA proteins were inherently higher in protein expression and enzyme affinity than the old lineage NA proteins and thus were able to tolerate the negative effects of the $\mathrm{H} 275 \mathrm{Y}$ mutation with a marginal loss of enzyme activity. As a result, the H275Y mutant $\mathrm{H} 1 \mathrm{~N} 1$ viruses of the new lineage had virus fitness equivalent to the wild-type viruses and were able to continue circulating, becoming the naturally resistant viruses. Further study revealed that 4 different amino acid substitutions played different roles in maintaining high protein expression and enzyme affinity of the new lineage NA proteins; the timeline of the sequential acquisition of the 4 substitutions was consistent with the timeline 
of emergence of the naturally resistant $\mathrm{H} 1 \mathrm{~N} 1$ viruses. The identified NA tolerance to the $\mathrm{H} 275 \mathrm{Y}$ mutation in the naturally resistant seasonal $\mathrm{H} 1 \mathrm{~N} 1$ viruses also had implication on the virus fitness of the $\mathrm{H} 275 \mathrm{Y}$ mutant $\mathrm{H} 1 \mathrm{~N} 1 / 2009$ viruses, as well as on the continuing surveillance monitoring of circulating pandemic H1N1 viruses.

Overall, both studies investigated in vitro and in vivo fitness of $\mathrm{H} 275 \mathrm{Y}$ mutant $\mathrm{H} 1 \mathrm{~N} 1$ viruses relative to their respective wild-type viruses, which were circulating in human beings at different times. These studies correlated the viral fitness of the $\mathrm{H} 275 \mathrm{Y}$ mutant viruses with the NA tolerance to the $\mathrm{H} 275 \mathrm{Y}$ mutation at protein level, and revealed that the NA tolerance to the $\mathrm{H} 275 \mathrm{Y}$ mutation was the molecular determinant of fitness of $\mathrm{H} 275 \mathrm{Y}$ mutant $\mathrm{H} 1 \mathrm{~N} 1$ viruses. The studies have implications on surveillance monitoring of the NA inhibitor resistance in circulating influenza viruses, which underlines the necessity of continuous monitoring of drug resistance incidence, as well as potential genetic and phenotypic changes of constantly evolving influenza viruses.

\section{Document Type}

Dissertation

\section{Degree Name}

Doctor of Philosophy $(\mathrm{PhD})$

\section{Program}

Biomedical Sciences

\section{Research Advisor}

Robert G. Webster, PhD

\section{Keywords}

Fitness, Influenza A virus, NA inhibitor, Resistance, Transmission

\section{Subject Categories}

Diseases | Enzymes and Coenzymes | Medical Immunology | Medical Microbiology | Medical Molecular Biology | Medical Sciences | Medicine and Health Sciences | Virus Diseases

\section{Comments}

Two year embargo expired May 2014 
The Molecular Basis of Fitness and Transmissibility of Neuraminidase Inhibitor Resistant Influenza A Viruses

\author{
A Dissertation \\ Presented for \\ The Graduate Studies Council \\ The University of Tennessee \\ Health Science Center
}

\begin{abstract}
In Partial Fulfillment
Of the Requirements for the Degree

Doctor of Philosophy

From The University of Tennessee
\end{abstract}

By

Susu Duan

May 2012 
Copyright (C) 2012 by Susu Duan. All rights reserved. 


\section{DEDICATION}

This dissertation is dedicated to my parents and my own family who have been always giving me unconditional love, indissoluble strength, endless inspiration. 


\section{ACKNOWLEDGEMENTS}

All of this would not have been possible had Dr. Robert G. Webster not taken me as his graduate student. I will always remember my first conversation with Dr. Webster about my rotating his lab and the first conversation with Dr. Webster at the day I officially joined the lab: "Work hard, young people." I have been doing nothing but following this simple instruction through all my studies during these years. I will be eternally grateful for Dr. Webster for having faith in me to take me as his student and mentoring my Ph.D study. I thank Dr. Webster for being my mentor in every way, for always giving me the biggest vision about the research and career, for teaching me to start strong and end strong a scientific story, for enlightening me with the most important and critical questions for now and for future in the world of influenza virology.

This dissertation would not have been completed in its present way without Dr. Elena G. Govorkova's supervision. The greatest appreciation is to Elena as my supervisor in all kinds of trainings: from introducing me the knowledge of the antiviral field, to helping me experiments in person, to supporting me to develop different projects, to teaching me to write manuscripts, to educating me to be a philosopher, and always reminding me to look pretty as a woman. I will always value all these precious training and experience.

Special deep thanks to Dr. Richard J. Webby for providing me different training opportunities in other aspects of influenza virology, for giving me different visions to see different questions. Especially many thanks for his biggest push to greatly move forward the second study in this thesis by adding new angles and much more depth.

Special thanks to my committee members, Dr. Paul Thomas, Dr. Michael Whitt and Dr. Tony Marion for giving me different perspectives to look the virology field, for always teaching me to think questions harder, deeper and more precisely.

Special thanks to Scott Krauss for his general support for all studies.

Special thanks to Dr. Subrata Barman and Dr. Charles Russell for kindly providing related experimental materials and working instructions.

Many thanks to a former postdoctor fellow in our lab, Dr. David Boltz, for being a good teacher and good friend to me, for introducing me the influenza genome analysis, for helping me to develop the methodology project and for giving me encouragement to through the ups and downs of the studies. Many thanks to another former postdoctor fellow, Dr. Hui-ling Yen, for also being a great teacher and friend to me, for her unique comments and inspiring discussion on many influenza questions and for her dear encouragement for me all the time. Many thanks to other current and former postdoctor fellows in our flu group for helping me with their respective expertise and those include Dr. Hassan, Zarakat, Dr. Henju Marjuki, Dr. Marriate Ducatez, Dr. Jacco Boon and other colleagues. 
Many thanks to our student fellows, Ali Ellebedy who is already Dr. Ellebedy now, and Tom Fabrizio, for their warmhearted help in all studies. Best wishes for Tom for his study.

Many thanks to Patrick Seiler for helping me through the big ferret experiments, which would not be that smooth without his help at each step. Thanks also to Dr. Tatiana Baranovich for the help with the ferret experiment.

Many thank to Kristi Prevost, Ashley Prevost, Heather Forrest, John Franks et al other colleagues for their warmhearted help in daily experiments.

Many thanks to Dr. Justin Bahl from Duke-NUS for his help with advanced phylogenetic analysis of H1N1 viruses and his different vision for the second study in the thesis.

Many thanks to Dr. Jiang Li from Hartwell center for his help and work to develop the methodology project.

Thanks to James Knowles for always being there to support us.

Thanks also to all the members of our amazing Flu Group at St. Jude Children's Research Hospital!

These works were funded by the National Institute of Allergy and Infectious Diseases, the National Institutes of Health, under contract number HHSN266200700005C, and the American Lebanese Syrian Associated Charities (ALSAC). 


\begin{abstract}
Neuraminidase (NA) inhibitors including oral oseltamivir and inhaled zanamivir are among the first line of defense against influenza virus infection. Development of resistance to NA inhibitors is a huge drawback for limited options for the control of influenza. During the first decade of NA inhibitor use, the detection rates of resistance to both NA inhibitors had remained low in circulating influenza viruses. However, the 2008 2009 season was marked by a radical increase of prevalence of oseltamvir resistance from $<1 \%$ to $>90 \%$ in worldwide surveillance in less than a year. The resistance was solely linked to NA $\mathrm{H} 275 \mathrm{Y}$ variants of seasonal H1N1 viruses, and they are referred as the naturally resistant viruses. A big question remains open about what fundamental molecular changes in the seasonal H1N1 viruses led to the surge of the naturally resistant viruses. When this question remained pending, a novel swine-origin H1N1 influenza virus emerged in Mexico at April 2009, soon spread worldwide replacing the seasonal influenza viruses including the naturally resistant viruses, and marked 2009 with the first influenza pandemic of the 21 st century. With sustainably increased worldwide use of NA inhibitors especially oral oseltamivir during the pandemic, the oseltamivir-resistant variants carrying H275Y NA mutation were isolated at low incidence from individuals receiving oseltamivir treatment and a few community clusters. In view of the high prevalence of naturally resistant seasonal H1N1 viruses in the immediate preceding season, there was an urgent need to characterize the transmissibility and fitness of oseltamivir-resistant pandemic H1N1/2009 viruses, although the resistance rates have remained low so far.
\end{abstract}

We first addressed the urgent question about pandemic viruses by investigating the transmissibility of a closely matched pair of pandemic H1N1/2009 clinical isolates, which only differed at the H275Y NA mutation in their genome, in the ferret model. We found that the H275Y NA mutant H1N1/2009 virus was not transmitted efficiently in ferrets via respiratory droplets, while it retained efficient transmission via direct contact. The wild-type H1N1/2009 virus was efficiently transmitted via both routes. The wild-type and the mutant viruses appeared to cause a similar disease course in ferrets without apparent attenuation of clinical signs. In the growth competition in a ferret, the H275Y mutant virus showed less growth capability than the wild-type virus. The NA of the $\mathrm{H} 275 \mathrm{Y}$ mutant virus showed reduced substrate-binding affinity and catalytic activity in vitro and delayed initial growth in MDCK and MDCK-SIAT1 cells. These findings may in part explain its less efficient transmission. The fact that the oseltamivir-resistant H1N1/2009 virus retained efficient transmission through direct contact underlines the necessity of continuous monitoring of drug resistance and characterization of more NA inhibitor-resistant variants of the pandemic H1N1 viruses.

We also sought to resolve the pending question about the naturally resistant seasonal H1N1 viruses by investigating the changes of different seasonal H1N1 viruses in terms of NA genetics, NA proteins attributes and virus fitness. We found that during the seasonal H1N1 virus evolution, two genetically diverged lineages of H1N1 viruses were circulating at different times. The NA protein phenotypes of the two lineages were 
naturally distinct in the levels of protein expression and enzyme affinity, and accordingly, the $\mathrm{H} 275 \mathrm{Y}$ NA mutation had differential effects on the NA proteins and virus fitness of the two lineages. The new lineage NA proteins were inherently higher in protein expression and enzyme affinity than the old lineage NA proteins and thus were able to tolerate the negative effects of the $\mathrm{H} 275 \mathrm{Y}$ mutation with a marginal loss of enzyme activity. As a result, the $\mathrm{H} 275 \mathrm{Y}$ mutant $\mathrm{H} 1 \mathrm{~N} 1$ viruses of the new lineage had virus fitness equivalent to the wild-type viruses and were able to continue circulating, becoming the naturally resistant viruses. Further study revealed that 4 different amino acid substitutions played different roles in maintaining high protein expression and enzyme affinity of the new lineage NA proteins; the timeline of the sequential acquisition of the 4 substitutions was consistent with the timeline of emergence of the naturally resistant H1N1 viruses. The identified NA tolerance to the $\mathrm{H} 275 \mathrm{Y}$ mutation in the naturally resistant seasonal $\mathrm{H} 1 \mathrm{~N} 1$ viruses also had implication on the virus fitness of the H275Y mutant H1N1/2009 viruses, as well as on the continuing surveillance monitoring of circulating pandemic H1N1 viruses.

Overall, both studies investigated in vitro and in vivo fitness of $\mathrm{H} 275 \mathrm{Y}$ mutant $\mathrm{H} 1 \mathrm{~N} 1$ viruses relative to their respective wild-type viruses, which were circulating in human beings at different times. These studies correlated the viral fitness of the H275Y mutant viruses with the NA tolerance to the $\mathrm{H} 275 \mathrm{Y}$ mutation at protein level, and revealed that the NA tolerance to the $\mathrm{H} 275 \mathrm{Y}$ mutation was the molecular determinant of fitness of $\mathrm{H} 275 \mathrm{Y}$ mutant $\mathrm{H} 1 \mathrm{~N} 1$ viruses. The studies have implications on surveillance monitoring of the NA inhibitor resistance in circulating influenza viruses, which underlines the necessity of continuous monitoring of drug resistance incidence, as well as potential genetic and phenotypic changes of constantly evolving influenza viruses. 


\section{TABLE OF CONTENTS}

\section{CHAPTER 1. GENERAL INTRODUCTION - NEURAMINIDASE INHIBITOR RESISTANCE IN INFLUENZA A VIRUSES: MOLECULAR MECHANISM AND VIRUS FITNESS ................................................................1 \\ Influenza Viruses and Influenza Disease ....................................................................

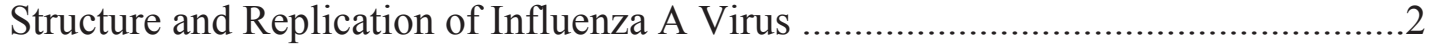

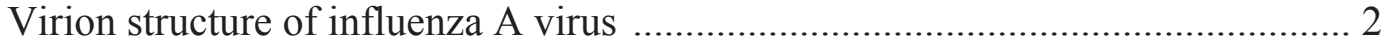

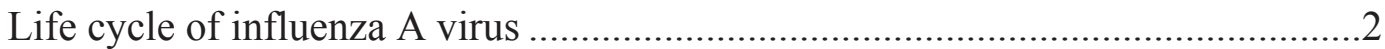 \\ Influenza A Virus Molecular Determinants on Pathogenesis and Transmission ........4 \\ Interventions of Influenza Infection ........................................................................6 \\ Vaccination and Antiviral Medication ..................................................................6

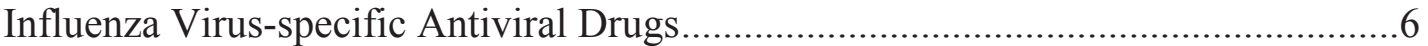 \\ Mechanism of Action of NA Inhibitors .................................................................. \\ NA Inhibitor Resistance in Influenza A Viruses - Mechnism, Prevalence and

 \\ NA Inhibitor Resistance in Human Seasonal Influenza A Viruses ..............................9 \\ Drug-selected resistance in human seasonal H1N1 and H3N2 influenza A

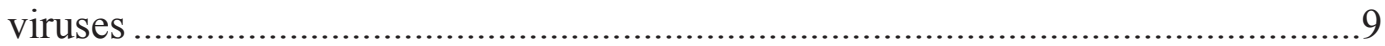 \\ Naturally-occurring resistance in human seasonal H1N1 influenza A viruses......12 \\ NA Inhibitor Resistance in 2009 Pandemic H1N1 Influenza A Viruses....................14 \\ NA Inhibitor Resistance in Human Infection of Highly Pathogenic H5N1

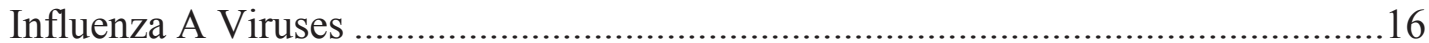

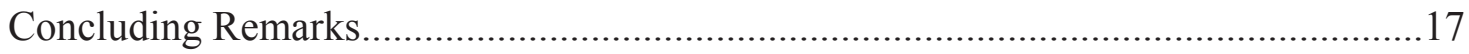

CHAPTER 2. THE VIRUS FITNESS AND TRANSMISSIBLITY OF AN OSELTAMIVIR-RESISTANT 2009 PANDEMIC H1N1 INFLUENZA A VIRUS. ..........................................................................................................................18

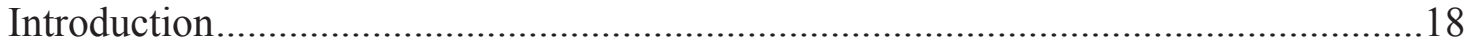

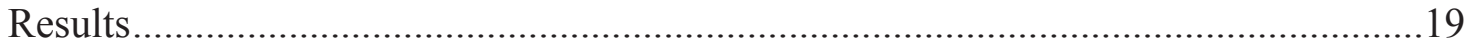

Sequencing and Phylogenetic Analysis of NA Genes ............................................19

NA Inhibitor Susceptibility and NA Enzyme Kinetics ...........................................21

Plaque Morphology and Growth Kinetics in Cells ..................................................21

Transmissibility among Ferrets via Direct-contact and Respiratory-droplets

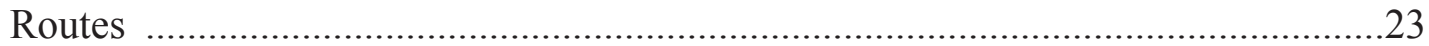

Co-inoculation with Oseltamivir-sensitive and -resistant H1N1/2009 Viruses ........27

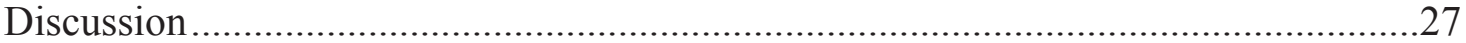

\section{CHAPTER 3. THE MOLECULAR BASIS OF NATURALLY-OCCURRING OSELTAMIVIR-RESISTANT H1N1 INFLUENZA A VIRUSES................................31}

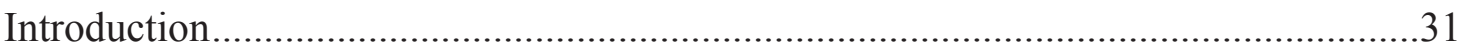

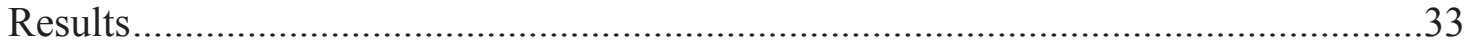

Evolution of NA Genes of Seasonal H1N1 Influenza Viruses ................................33

Transmissibility of Different Seasonal H1N1 Viruses in Ferrets .............................39

Transmissibility of wild-type and H275Y mutant BR07-like viruses in ferrets....39 
Transmissibility of reverse-genetics BR/59/07 viruses carrying H275Y NA mutation or/and D354G mutation ..................................................................4

Transmissibility of wild-type and H275Y mutant NC99-like viruses in ferrets....45

Growth Capability of Different Seasonal H1N1 Viruses in Cell Culture....................46

NA Enzyme Activity, Protein Expression and Enzyme Kinetics of Different

Seasonal H1N1 Viruses in vitro .........................................................................48

Generation and characterization of epitope-tagged recombinant NA proteins......48

Enzyme activities of recombinant NA proteins of $\mathrm{H} 1 \mathrm{~N} 1$ viruses in vitro ..............52

Expression levels of recombinant NA proteins of H1N1 viruses in cells...............52

NA enzyme kinetics of H1N1 viruses in vitro ....................................................54

Effects of the Single Amino Acid Substitutions in BR07-NA Gene (from

BR07-like to NC99-like) on Protein Function and Expression ..................................55

Effects of NA variants of different enzyme activities on plaque morphology

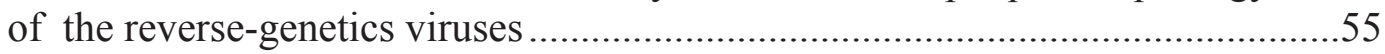

Effects of the single amino acid substitutions (from BR07-like to NC99-like)

in BR07-NA gene on plaque morphology of the reverse-genetics viruses............56

Effects of the single amino acid substitutions (from BR07-like to NC99-like)

in BR07-NA gene on protein function and expression.

Effects of the Reciprocal Single Amino Acid Substitutions in GA06-NA Genes

(from NC99-like to BR07-like) on Protein Function and Expression ........................61

Determination of the Timeline of Fixations of the Four Molecular Markers

during Evolution of N1 NA Genes

Effects of Sequential Addition of the Four Molecular Markers into GA06-NA

Genes on Protein Function and Expression ............................................................65

Determination of NA Tolerance to the H275Y Mutation in 2009 Pandemic

H1N1 Viruses

NA gene comparison between the pandemic and seasonal H1N1 viruses ............70

NA activity, protein expression and enzyme affinity of the wild-type and

$\mathrm{H} 275 \mathrm{Y}$ mutant pandemic $\mathrm{H} 1 \mathrm{~N} 1$ viruses in vitro .....................................................70

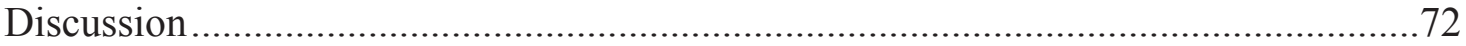

CHAPTER 4. METHODS ….........................................................................................81



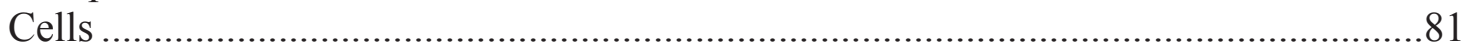

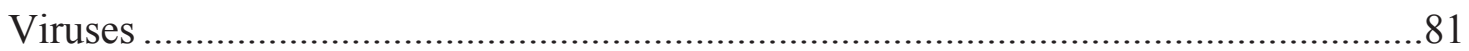

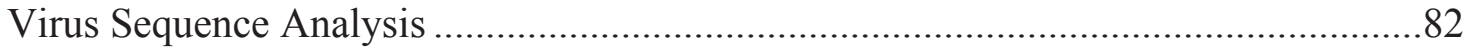

Plasmid Construction and Site-directed Mutagenesis .................................................8

Generation of Reverse-genetics Influenza Viruses......................................................82

Generation and Detection of Recombinant NA Proteins ..............................................83

Determination of Infectivity of Influenza Viruses.......................................................83

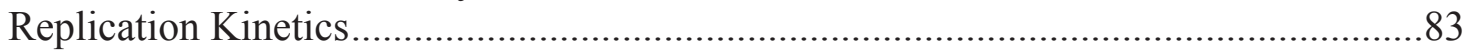

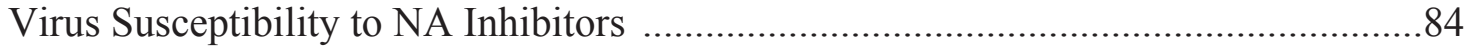

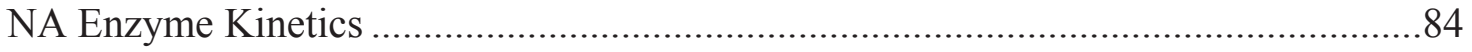

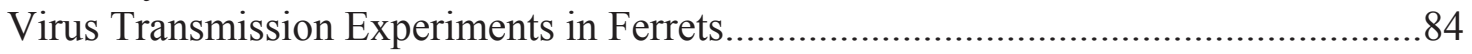

Collection and Titration of Nasal Wash Samples .......................................................85

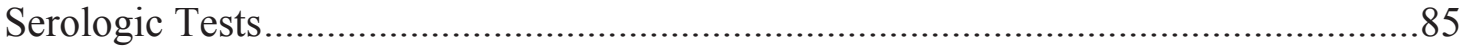


Quantitative Single Nucleotide Polymorphism Assay...................................................85

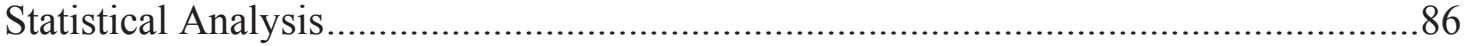

LIST OF REFERENCES ...................................................................................................87

APPENDIX. NOVEL GENOTYPING AND QUANTITATIVE ANALYSIS

OF NEURAMINIDASE INHIBITOR RESISTANCE-ASSOCIATED

MUTATIONS IN INFLUENZA A VIRUSES BY SINGLE-NUCLEOTIDE

POLYMORPHISM ANALYSIS ....................................................................................100

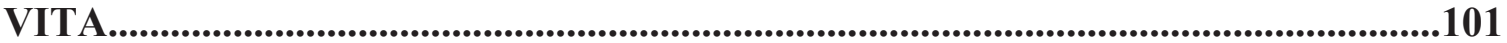




\section{LIST OF TABLES}

Table 2-1. Neuraminidase enzymatic properties of the H1N1 influenza viruses .........20

Table 2-2. Clinical signs, virus replication and sero-conversion in inoculated donor



Table 2-3. Clinical signs, virus replication, and seroconversion in contact ferrets .......26

Table 3-1. Comparison of the amino acid consensus sequences of NA genes of human seasonal H1N1 viruses of different lineages .................................35

Table 3-2. Agreement of the NA genes of the H1N1 viruses with the respective NA censuses sequences of human H1N1 viruses of different lineages at the 10 amino acid positions plus the 275 position ....................................... 37

Table 3-3. Detection of virus replication and sero-conversion in ferrets during transmission of the H1N1 influenza viruses 


\section{LIST OF FIGURES}

Figure 2-1. Plaque morphology and replication kinetics of two H1N1/2009 influenza viruses in MDCK and MDCK-SIAT1 cells.....

Figure 2-2. Transmissibility of the two H1N1/2009 influenza viruses among ferrets ...24

Figure 2-3. Co-infection with oseltamivir-sensitive and -resistant H1N1/2009 influenza viruses .28

Figure 3-1. Evolution of NA genes of human seasonal H1N1 influenza viruses 34

Figure 3-2. The structure of NA monomer (N1 subtype) showing the 275 position and the 10 positions at which the amino acid substitutions differentiate the NAs of NC99-like and BR07-like.

Figure 3-3. Transmissibility of the seasonal H1N1 influenza viruses of two lineages in ferrets

Figure 3-4. Growth capability of different H1N1 viruses in NHBE cells

Figure 3-5. Generation and characterization of epitope-tagged recombinant NA protein of $\mathrm{BR} / 59 / 07$ virus

Figure 3-6. The cell surface protein accumulation of C-terminal tagged BR07-NA protein

Figure 3-7. The NA enzyme activities, protein expression levels and enzyme Km values of different seasonal H1N1 influenza viruses.

Figure 3-8. The plaque sizes of $\mathrm{rgBR} / 59 / 07$ viruses containing different NA gene variants

Figure 3-9. The plaque morphologies of $\mathrm{rgBR} / 59 / 07$ viruses of single amino acid residue substitutions in the NA gene

Figure 3-10. Effects of five different single amino acid substitutions in the BR07-NA genes on NA enzyme activity, km value and protein expression

Figure 3-11. Effects of four different single amino acid substitutions in the GA06-NA genes on plaque size of reverse-genetics viruses, NA enzyme activity, NA Km value and NA protein expression

Figure 3-12. The plaque morphology of $\operatorname{rgBR} / 59 / 07$ viruses by using variant GA06-NA genes with different single amino acid substitutions 
Figure 3-13. The timeline of fixation of the 6 amino acid residue substitutions in the

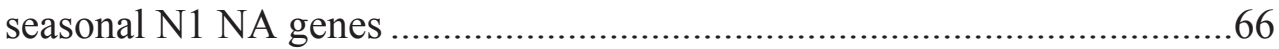

Figure 3-14. Effects of sequential addition of four amino acid substitutions in the GA06-NA genes on plaque size of reverse-genetics viruses, NA enzyme activity, NA Km value and NA protein expression ................................67

Figure 3-15. The plaque morphologies of $\operatorname{rgBR} / 59 / 07$ viruses by using variant GA06-NA genes with sequential addition of the 4 amino acid substitutions

Figure 3-16. The NA activities, protein expression and enzyme $\mathrm{Km}$ values of the 2009 pandemic $\mathrm{H} 1 \mathrm{~N} 1$ viruses. 


\section{LIST OF ABBREVIATIONS}

$\begin{array}{ll}\text { BHK } & \text { Baby Hamster Kidney Cells } \\ \text { BR07 } & \text { A/Bribane/59/2007 (H1N1) } \\ \text { BR/59/07 } & \text { A/Bribane/59/2007 (H1N1) } \\ \text { CDC } & \text { US Centers for Disease Control and Prevention } \\ \text { DC } & \text { Direct Contact } \\ \text { DM/09 } & \text { A/ Denmark/524/2009 (H1N1) or A/ Denmark/528/2009 (H1N1) } \\ \text { DM/524/09 } & \text { A/ Denmark/524/2009 (H1N1) } \\ \text { DM/528/09 } & \text { A/ Denmark/528/2009 (H1N1) } \\ \text { GA06 } & \text { A/Georgia/17/2006 (H1N1) or A/Georgia/20/2006 (H1N1) } \\ \text { GA/17/06 } & \text { A/Georgia/17/2006 (H1N1) } \\ \text { GA/20/06 } & \text { A/Georgia/20/2006 (H1N1) } \\ \text { HA } & \text { Hemagglutinin } \\ \text { HI } & \text { Hemagglutination Inhibition } \\ \text { HW/28/08 } & \text { A/Hawii/28/2008 (H1N1) } \\ \text { M1 } & \text { Matrix Protein 1 } \\ \text { M2 } & \text { Matrix Protein 2 } \\ \text { MDCK } & \text { Madin-Darby Canine Kidney Cells } \\ \text { MDCK-SIAT1 } & \text { Madin-Darby Canine Kidney Cells -human 2,6-sialyltransferase } \\ \text { MEM/13/06 } & \text { A/Memphis/13/2006 (H1N1) } \\ \text { MI/03/02 } & \text { A/Mississipii/03/2002 (H1N1) } \\ \text { MOI } & \text { Multiplicity of Infection } \\ \text { NA } & \text { Neuraminidase } \\ \text { NC99 } & \text { A/New Caledonia/20/1999 (H1N1) } \\ \text { NHBE } & \text { Normal Human Bronchial Epithelial Cells } \\ \text { NJ/15/07 } & \text { A/New Jersey/15/2007 (H1N1) } \\ \text { NY/1692/09 } & \text { A/ New York/1692/2009 (H1N1) } \\ \text { NY/3467/09 } & \text { A/ New York/3467/2009 (H1N1) } \\ \text { pfu } & \text { Plaque Forming Unit } \\ \text { pH1N1 } & \text { Pandemic H1N1 } \\ \text { RD } & \text { Respiratory Droplet } \\ \text { Rg } & \text { Revrse-genetics } \\ \text { RNA } & \text { Ribonucleic Acid } \\ \text { RNP } & \text { Ribonucleoprotein } \\ \text { SNP } & \text { Single Nucleotide Polymorphism } \\ \text { TCID } 50 & \text { 50\% Tissue Culture Infectious Dose } \\ \text { WHO } & \text { World Health Organization } \\ & \end{array}$




\section{CHAPTER 1. GNERAL INTRODUCTION - NEURAMINIDASE INHIBITOR RESISTANCE IN INFLUENZA A VIRUSES: MOLECULAR MECHANISM AND VIRUS FITNESS}

\section{Influenza Viruses and Influenza Disease}

Influenza viruses are among the most important human-inflicting pathogens. Influenza viruses are enveloped viruses with single-stranded negative-sense segmented RNA genome, belonging to the members of the orthomyxoviridae family. Influenza viruses can be classified into A, B, C types. Human influenza viruses are mainly A and B type. Influenza B is separated into Yamagata and Victoria lineages. In influenza A viruses, two virus surface glycoprotein hemagglutinin (HA) and neuraminidase (NA) determine and differentiate their antigenic subtypes into 16 HA subtypes and 9 NA subtypes (1). All these influenza A subtypes can be found in their natural reservoir aquatic birds (2). Only three HA subtypes (H1, H2, H3) and two NA (N1, N2) subtypes have been found to cause efficient infection in human populations, including human $\mathrm{H} 1 \mathrm{~N} 1, \mathrm{H} 2 \mathrm{~N} 2$ and H3N2 subtype viruses. In addition, occasional human cases of the infection with avian influenza A viruses of H5, H7 and H9 subtypes have been reported (3-5). In circulating human influenza viruses, HA and NA surface proteins constantly evolve to escape the accumulated herd immunity pressure against the two proteins by acquiring mutations at their antigenic sites known as antigenic drift. In addition, the reassortment ability between two different influenza virus strains can generate a new strain of virus which possesses distinct surface proteins from their parental viruses, known as antigenic shift.

Influenza viruses cause an acute contagious disease in the respiratory tracts influenza. Every year, human seasonal influenza viruses which are mainly co-circulating influenza A H1N1 and H3N2 subtypes and influenza B type, cause annual influenza epidemics. The typical clinical symptoms of human influenza virus infection include coughing, sneezing, sore throat and fever. Severe disease including pneumonia and other systematic symptoms are normally limited to the susceptible populations such as immunocompromised patients, young children and elderly people. Although the seasonal influenza outbreaks normally tend to be mild, they still cause thousands of deaths and millions of hospitalizations annually. Periodically, new strains of influenza viruses can emerge from different species and rapidly spread into human beings, causing world-wide outbreaks of infection - influenza pandemics. Historically, there are serious pandemics in the 20th century with numerous death caused by different influenza viruses H1N1, H2N2, and H3N2, respectively (2). Most recent example is the $2009 \mathrm{H} 1 \mathrm{~N} 1$ influenza virus pandemic, the first pandemic in the 21 st century caused by a novel swine-origin influenza virus of H1N1 subtype $(6 ; 7)$. The most confirmed human cases of H1N1/2009 influenza have been uncomplicated and mild, but the number of cases and affected countries was unprecedented. In addition, human infection of avian influenza viruses had variable clinical outcomes, with symptoms involving typical influenza symptoms and other, pneumonia, rhihnorrhea, diarrhea, hypoxemia and leucopenia systematic symptoms (8). The human cases of avian H5N1 influenza virus infection, which sporadically occurred at 
South East Asia and Middle East Africa (9), are especially associated with extremely high mortality and morbidity. Although transmission of $\mathrm{H} 5 \mathrm{~N} 1$ influenza viruses from avian species to humans is currently inefficient, the novel antigenicity of the viruses for human populations and the highly fatal outcome of the infection raised huge concern about their potential to become the next pandemic virus and the severity of the potential pandemic.

\section{Structure and Replication of Influenza A Virus}

Virion structure of influenza A virus. Virions of Influenza A virus are lipid-enveloped particles, typically displaying roughly spherical shape or long filament shape $(1 ; 10)$. The virions have three transmembrane proteins including two surface glycoproteins HA and NA and one membrane-embedded M2 protein. HA protein is the most abundant envelope protein (80\%). They are rod-shaped trimer molecules with $\mathrm{C}$ terminus forming a membrane anchor inserted into viral membrane and $\mathrm{N}$ terminus forming a globular head projected away from viral surface like a spike. NA protein is the second major envelope protein (17\%) and is cluster together as patches on the viral membrane. NA protein is mushroom-shaped tetramer molecule with a short N-terminal cytoplasmic tail and transmembrane region as anchor for the stalk and head. M2 protein has very minor abundance among envelope proteins and it is also a tetramer molecule which forms ion channels spanning the envelope. On the interior side of the virion lipid envelope, a matrix formed by M1 protein holds 8 viral ribonucleoprotein (vRNP) complexes in each virion. Each vRNP complex is made up of one RNA gene segment coated by nucleoproteins (NP) and associated with a polymerase complex of PB1, PB2 and PA proteins. The virions also contain Nanostructure Protein 2 (NS2), sometimes also called nuclear export protein (NEP). The 8 genome segments of influenza A viruses encode for 10 viral proteins. Six segments encode HA, NA, NP, PB1, PB2, PA proteins respectively, and the mRNAs of 2 segments, $M$ and NS segments can be alternatively spliced into M1/M2 and NS1/NS2 genes respectively. Among the 10 viral proteins, except for NS1 protein, other 9 proteins are incorporated into the virus particles during virus replication. In some influenza $A$ viruses, the +1 reading frame of $\mathrm{PB} 1$ gene can encoded an additional protein PB1-F2 of up to 90 amino acid residues (11). PB1-F2 proteins are truncated in some influenza $A$ viruses, due to the frequent appearance of stop codon in the alternative reading frame of PB1 gene. Each protein function differently and concertedly during replication cycle of influenza A viruses.

Life cycle of influenza A virus. Concisely, the replication cycle of influenza A virus in cells can be divided into several phases (10): virus entry into host cells, import of vRNPs into the nucleus, transcription and replication of viral genome, export of nascent vRNPs from the nucleus, assembly and budding of progeny virions, and finally release of the progeny viruses from infected host cell surface. Each phase is involved with multiple molecular events mediated by different host and viral factors and a lot of precise details in these events are still under investigation. 
Influenza A virus replication start from the entry of virus particles into host cells. This phase is involved several steps including virus attachment, endocytosis of virus particle, fusion of viral envelope with endosomal membrane and uncoating of virus particle within endosome $(1 ; 10)$. The surface glycoprotein HA plays several different critical roles during these steps. The HA protein is synthesized as a single polypeptide precursor HA0. For most influenza viruses, the HA0 must be cleaved by host proteases into HA1 and HA2 subunits which are linked by disulphide bonds. HA1 subunit contains receptor binding domain, and HA2 subunit contains transmembrane domain and a fusion peptide. Influenza A virus entry starts from virus attachment to host cell surface by the interaction of the HA receptor binding domain with the sialic acid containing-receptors at host cell surface. Upon binding to the cell surface, the virus is internalized by receptor-mediated endocytosis. Being inside an endosome, the virus enters into the host cell. When the endosomal $\mathrm{pH}$ is decreased to $\sim 5$ by action of an $\mathrm{H}+$ pump on the endosomal membrane, the low $\mathrm{pH}$ induce an irreversible confirmation change of the whole cleaved HA molecule, which expose and realign the fusion peptide in the HA2 subunit. The realigned fusion peptide bring the viral and endosomal membranes into contact with each other and insert itself into the endosomal membrane. The action of more than two fusion peptides form the fusion pores in the membrane of the virion, enabling release of the content of the virion into cell cytoplasm. The acidic environment in the endosome also open M2 ion channels in the viral membrane, allowing the influx of $\mathrm{H}+$ ions from endosome into the virion. The acidification of the virion disrupts vRNPs binding to M1 proteins, leading to the release the free vRNPs into cyctoplasma, which complete the uncoating process.

After the vRNPs are released into the cytoplasm, they are to be imported into the nucleus, where they serve as templates for transcription and replication of virus genome $(1 ; 10)$. All proteins in vRNP complex including NP, PB1, PB2 and PA possess nuclear localization signals (NLSs), which interact with cellular nuclear import machinery and mediate the vRNP import into nucleus. Once in the nucleus, in order for synthesis of the viral proteins, each negative-sensed RNA segment is transcribed into messenger RNA (mRNA), which is capped and polyadenylated positive-sensed RNA for protein translation. For replication of the viral genome, each negative-sensed RNA segment is firstly transcribed into an exact positive-sensed complementary RNA (cRNA) copy, then in turn the cRNA serve as template for synthesis of more copies of negative-sensed viral RNA (vRNA). All these transcription and replication of viral RNA segments are catalyzed by the same viral polymerase complex, an RNA-dependent RNA polymerase complex made up with three subunits PB1, PB2 and PA. The viral mRNAs are transported to the cytoplasm and serve as templates for translation of viral proteins. NS1 protein is believed to block the transportation of host cell mRNAs thus facilitating the viral mRNA process. Newly synthesized M1, NP and NS2 proteins are imported back into the nucleus for assembly and transportation of new vRNPs. Following virus replication, newly formed vRNPs are assembled in the nucleus and are to be exported into the cytoplasm. It is believed that M1 protein and NS2 protein are involved in this process by masking the NLS in the proteins of vRNPs and also interacting with nuclear export machinery respectively. 
After the vRNPs have been exported into cytoplasm, they are to be packed with other viral proteins into virus particles to leave the cells $(1 ; 10)$. This process occurs at the apical plasma membrane of polarized cells and involves with multiple events including assembly of different viral components, package of all 8 segments, membrane budding and release of nascent virons. The three transmembrane proteins are assembled into trimers (HA) or tetramers (NA and M2) in cell endoplasmic reticulum, and then they are directed to assembly sites and are further incorporated into cell membrane by the signals residing in the transmembrane domains of HA and NA proteins. Other viral components including viral segments also reach the assembly sites and are poised for virion budding. Virus budding is seen as the extrusion of cell membrane along with all the virus components in its inner core and eventual formation of virus particles after the fission from the cell membrane. M1 is believed as the driving force behind this process. After completion of virus budding, the virus particles are attached to the infected cell surface due to HA binding to the sialic acid-containing receptors, so the progeny virions cannot leave cell membrane without active release by the action of NA. The enzyme activity of NA proteins cleaves the sialic acid residues from glycoproteins and glycolipids on the cell membrane, thereby releasing the progeny virions into extracellular environment to infect new host cells.

\section{Influenza A Virus Molecular Determinants on Pathogenesis and Transmission}

Each viral protein of influenza virus plays different and indispensable role in virus life cycle and they are tightly regulated and coordinated with host factors to cause an effective virus infection in a host. Primarily all the viral proteins contribute to successful infection of influenza A virus in hosts, although the precise action mechanism of each viral protein on the virus pathogenesis and transmission in hosts is still to be further explored. Studies of different subtype influenza A viruses of different properties have identified several dominant viral molecular determinants on virus pathogenesis and transmission $(2 ; 12 ; 13)$.

Two major functions of HA protein, receptor-binding and fusion activity, both contribute to virus transmission and pathogenesis $(2 ; 12 ; 13)$. Firstly, HA proteins have specificity for recognition of host cell receptors, making HA a determinant for influenza virus host range. Now, it is generally believed that HA proteins of human influenza virus isolates preferentially binds to $\alpha 2,6$-linked sialic acids, while HA proteins of avain virus isolates preferentially binds to $\alpha 2,3$-linked sialic acids (14). Mainly $\alpha 2,6$-linked sialic acids are found in human upper respiratory tract, the primary site of influenza infection, which impose a restriction for efficient transmission of avain influenza virus into human beings. Accordingly, virus possession of HA recognizing human-type receptors is also seen as a pre-requisite for sustained human-to-human transmission of the virus. Secondly, HA precursor cleavage is essential for viral infectivity and cleavability of HA precursor is a restriction factor for effective virus replication. The HA proteins of most of influenza A viruses only posses a single Arg residue at the cleavage site, which must be cleaved by host cell proteases in respiratory or intestinal organs, thus the virus replication is restricted locally within these organs. In contrast, the HA proteins of highly pathogenic 
$\mathrm{H} 5$ or $\mathrm{H} 7$ viruses possess multi-basic amino acids at the cleavage site (15), which allows their proteolytic cleavage by universal unbiquitous proteases during HA peptide post-translational modification and leads to severe systemic infection. Therefore, the multi-basic HA cleavage motif is seen as a virulence factor for influenza virus.

The replication capability of polymerase complex of influenza A virus contributes to virus pathogenicity by directly controlling virus growth in hosts. Several mutations such as E627K and D701N in PB2 subunit are associated with the polymerase replication ability, thus virus pathogenicity and transmission $(2 ; 12 ; 13)$. PB2 E627K mutation in the avian $\mathrm{H} 5 \mathrm{~N} 1$ influenza viruses can enhance the polymerase replication ability in $33^{\circ} \mathrm{C}$, but not at higher temperature $37^{\circ} \mathrm{C}$ and $41^{\circ} \mathrm{C}(16) .33^{\circ} \mathrm{C}$ is the approximate temperature of mammalian upper respiratory tract where human influenza viruses replicate, whereas $41^{\circ} \mathrm{C}$ is the approximate temperature of avian intestinal tract where avian influenza viruses replicate. Therefore, avian H5N1 influenza viruses with PB2 E627K mutation had increased pathogenicity in mammalians (17). In contrast, PB2 K627E mutation in the human influenza A viruses reduces virus transmission in mammalian animal models due to reduced replication in their upper respiratory tracts (18).

PB-1 F2 is a small protein of up to 90 amino acid residues encoded by an alternative reading frame of PB1 gene and it can be truncated at different lengths by different location of stop codon in the alternative reading frame. PB1-F2 preferentially localizes to mitochondria membrane in the infected cells and has a pro-apoptotic function (11). PB1-F2 also can enhance the virus polymerase activity in vitro (19). An S66N mutation in PB1-F2 mutation was found to be responsible for the high virulence of both 1918 pandemic and avian H5N1 influenza viruses (20). Therefore, PB1-F2 is also known as virulence factor of influenza viruses.

Pathogencity of influenza A viruses also depends on their ability to evade host immune response, especially the first line innate response as influenza viruses only cause acute infection. NS1 protein is found as an interferon antagonist to suppress host innate response to viruses (21). NS1 prevents activation of certain transcription factors thus also the following stimulation of interferon production. The NS1 proteins of highly pathogenic $\mathrm{H} 5 \mathrm{~N} 1$ viruses are associated with induction of high levels of pro-inflammatory cytokines in hosts, which was linked to the severe inflammation and high morbidity and mortality caused by the viruses $(2 ; 22)$. Several mutations such as D92E and P42S in NS1 protein of the H5N1 viruses have been linked to virus virulence $(23 ; 24)$.

The enzyme activity of NA function mainly functions to actively release progeny virus particles from infected cell surface. NA is also believed to function to cleanse the respiratory tract environment such as mucus and cell surface for virus entry by removing the sialic acid from host glycoproteins or glycolipids. In addition, NA also can prevent virus particle aggregation and allow virus spread (25). Efficient influenza A virus replication requires an optimal functional balance between HA sialic acid-binding activity and NA receptor-destroying activity. The balance can be disturbed by reassortment between different virus strains, interspecies transmission etc events and the 
changes of function NA and their impacts on virus infectivity during these events have been described $(26 ; 27)$. Especially during antiviral treatment, influenza virus can adapt to NA inhibitor drugs by generation of resistant viruses of NA mutations. How these NA mutations cause resistance to NA inhibitors and how they affect NA function and fitness of the mutant viruses including virus pathogenesis and transmissibility is the focus of this review as discussed below.

\section{Interventions of Influenza Infection}

\section{Vaccination and Antiviral Medication}

Vaccination is one of the effective interventions for influenza infection, primarily for preventing infection and reducing the morbidity and mortality associated with the influenza infection. Seasonal influenza vaccines, either the trivalent inactivated virus vaccine or the live attenuated virus vaccine include contemporary H1N1 and H3N2 influenza A virus and influenza B as the three antigen components. The three antigen components have to be evaluated annually based on the contemporary prevalence of the circulating viruses, and are subject to reformulation when the antigenicity of prevalent circulating viruses changes. Thus, the efficacy of vaccination is largely determined by the matches between circulating viruses and selected vaccine strains and can be significantly reduced by mismatches. Additionally, the efficacy of vaccination is also limited by the adequacy of host immune response against the vaccine antigens, the complication of the pre-existing immunity mounted in individuals especially adults. More importantly, in the scenario of a pandemic which are caused by emergent influenza viruses with new antigentic features, there is considerable delay time, normally at least 6 months, before a sufficient amount of the new antigenically-matching vaccines can be produced.

Thus, antiviral medication provides another option for the control and management of acute influenza infection. The goal of antiviral treatment is to decrease the disease associated symptoms, complications, mortality etc. Specific anti-influenza drugs have also been used for both prophylactic and therapeutic treatments of influenza infection during seasonal epidemics. Antiviral medication is particularly important at the beginning of a fast-spreading pandemic, and it is even likely to become the only first-line control strategy before the antigenically-matching vaccine is available. The beneficial effects by the antiviral medication on patients including reducing virus shedding duration, illness symptoms and functional disability caused by the illness, although the magnitude of the efficacy varies, depending on the drugs used, the populations treated, the viruses infected and the timing of the treatment initiation etc.

\section{Influenza Virus-specific Antiviral Drugs}

At present, there are two classes of FDA-approved antiviral drugs specific for influenza viruses, M2 blockers and NA inhibitors. M2 blockers including amantadine and 
rimantadine were the earliest class of specific anti-influenza compounds. Amantadine was approved by FDA as early as at 1963 and rimantadine at 1993. This class of drugs targets the M2 ion channel located in the viral membrane. Binding of the drugs in the channels blocks virus uncoating thus prevent its following replication inside the cell. M2 blockers are only effective against influenza A virus. They are effective for prophylaxis and therapeutic treatments of influenza infection and exert clinic benefit including decreased duration of virus shedding and disease symptoms in patients (28). The resistance to M2 blockers are developed during treatment and conferred by amino acid residues changes in the M2 ion channel, most notably at S31N. The prevalence of the M2 blocker resistance is a serious problem for this class of drugs: to date, it has been reported of $>90 \%$ resistance rate in seasonal $\mathrm{H} 3 \mathrm{~N} 2$ viruses, overall $15 \%$ rate in seasonal $\mathrm{H} 1 \mathrm{~N} 1$ viruses, $100 \%$ rate in all characterized 2009 pandemic H1N1 viruses, and varied rates including as high as 90\% in certain clades of avian H5N1 viruses (29) (30;31). The high-level circulation and rapid spread of the resistant influenza viruses in the community have compromised the usefulness of the M2 blockers.

Another class of specific influenza virus-specific antivirals is the NA inhibitors, including oral oseltamivir and inhaled zanamimir which were approved by US FDA at 1999 for treatment of influenza infection (32). The third investigational NA inhibitor, peramivir developed as intravenous formulation, has been licensed only in Japan, but is in ongoing clinic trails in US now (28). Based on its promising efficacy, it was warranted by US FDA with an emergency use authorization for treatment of 2009 pandemic influenza virus infection in certain patients (33). NA inhibitors now are the first line of specific anti-influenza drugs due to the high prevalence of resistance to the M2 blockers in human influenza A viruses and the lack of activity against influenza B viruses of the M2 blockers.

\section{Mechanism of Action of NA Inhibitors}

NA inhibitors target the NA enzyme, one of the membrane glycoproteins presented at the surface of the influenza virus particle. Influenza virus NA functions to cleaves the $\alpha-2,3$ - or $\alpha-2,6-$ ketosidic linkage between a terminal sialic acid residue and an adjoining sugar residue $(28 ; 34)$. In the influenza virus replication cycle, by removing the sialic acids residues from the membrane surfaces of infected cells, NA destroys the association between infected host cells with HA proteins, thus facilitating the release the nascent viruses and allowing the progeny viruses to infect new host cells $(28 ; 34)$.

All type/subtypes of influenza viruses have abundant NA proteins in their membranes, and the main structure and critical amino acid residue at the enzyme active site are highly conserved among all NA stubypes of influenza viruses, although the NA protein constantly evolves under immunity pressure and continuously changes the amino acid residue composition. 19 conserved residues were identified at the NA active site: including 8 catalytic residues (R118, D151, R152, R224, E276, R292, R371, and Y406; N2 numbering) which directly interact with the sialic acid substrate during the enzyme reaction, and 11 framework residues (E119, R156, W178, S179, D/N198, I222, E227, 
H274, E277, N294, and E425) which support structure and function of the active site (35). NA inhibitors were all developed through rational drug designs based on the knowledge of the crystallographic structure of the NA complex with sialic acid $(36 ; 37)$.

All three NA inhibitors, oseltamivir, zanamivir and peramivir are analogues of sialic acid, the natural substrate of the NA enzyme (36). NA inhibitors competitively bind to the NA active site, and thus block the binding of sialic acid, its natural substrate. Owning to the essential role of NA for release of progeny virions from the infected host cells, the inhibition of NA prevents virus spread and future replication in adjacent cells in the host. The mode of action of the NA inhibitor was proved by electron microscopy imaging (25). In the electron microscopy images, in the absence of inhibitor, separate rounded virions were observed on the surface of the cells; in the presence of oseltamivir carboxylate, clumped flattened virons were observed to attach to the cell surface, suggesting the failure to detach from the cell surface (25). As the neuraminidase active site is highly conserved in all types of influenza viruses, the NA inhibitors are effective against all human, avian and animal influenza viruses (25). The antiviral activity of the NA inhibitors have been demonstrated and proved in NA enzyme inhibition assays in vitro, infectivity reduction assays in cell culture, experimental animal models and human treatment experience $(25 ; 30)$.

\section{NA Inhibitor Resistance in Influenza A Viruses - Mechanism, Prevalence and Fitness}

The resistance to NA inhibitor can greatly reduce the therapeutic efficacy and disable the limited infection control options for influenza, so the susceptibility of the circulating influenza viruses to NA inhibitors have been closely monitored in clinical observation studies and surveillance studies.

Assessment of susceptibility of influenza viruses to NA inhibitors is primarily performed in functional NA inhibition assay in vitro and the NA genes of resistant viruses were further sequenced to understand the mechanism of resistance (38). The susceptibility of the virus to the drug can be firstly defined by the $50 \%$ percentage of inhibition concentration values $\left(\mathrm{IC}_{50}\right)$ in $\mathrm{NA}$ inhibition assays. $\mathrm{IC}_{50}$ values vary by the type NA inhibition assay, influenza type and NA inhibitors tested, so the folds of change of $\mathrm{IC}_{50}$ values have been set as the criteria defining NA inhibitor resistance which is $\mathrm{IC}_{50}>10$-fold means of the reference type/subtype sensitive influenza viruses to the drug (38). Thus, a significant increase of the $\mathrm{IC}_{50}$ value of a virus to a drug compared to the sensitive viruses can be defined as resistance or reduced susceptibility, although there is no clear-cut standard to differentiate both terms. Before the NA inhibitors were approved for clinical practice, over 1000 influenza virus isolates were examined for their susceptibility to NA inhibitors and they were found to be naturally sensitive (25).

When influenza viruses resistant to NA inhibitors are identified, the resistance phenotype can be mapped to NA mutations by genotyping methods. The known resistance markers have been mapped to either the catalytic residues or framework 
residues of NA active site. Apart from characterization of resistant virus genotype and phenotype, the impacts of resistance-associated mutations on the function of NA protein can be evaluated by enzyme kinetics, such as $\mathrm{Km}, \mathrm{Ki}$ and Vmax etc parameters. $\mathrm{Km}$ is the dissociation constant for enzyme binding to the substrate, which is intrinsic to the enzyme and independent of the amount of protein presented in the reaction; Likewise, Ki is the dissociation constant for enzyme binding to the enzyme inhibitor; Vmax reflects catalytic capability of the enzyme active site and is dependent on both the enzyme turnover rate (Kcat) and the amount of enzyme presented in the reaction.

After resistance genotype and phenotype of influenza viruses are characterized, the fitness of resistant viruses is of particular interest for further investigation. Viral fitness is defined as the degree of virus adaptation to a given environment or its relative ability to produce infectious progeny in a host (39). Originally, a growth competition experiment between different viruses is adopted to define the fitness degree of a virus relative to another virus (39) (40). However, due to the difficulty to quantitatively differentiate different genotypes in mixed populations, growth competitions cannot be always easily realized. As an alternative, now a comparative experiment using different viruses under the same experimental settings generally can be used to describe the fitness level of a virus relative to other viruses. The virus fitness can be evaluated in vitro or in vivo, such as virus growth kinetics and capability in different cell cultures, virus plaque morphology in cells and virus growth, pathogenicity and transmissibility in animal models etc infectivity parameters.

In the follow sections, the mechanism and prevalence of the resistance to NA inhibitors in influenza A viruses is discussed, which includes human seasonal influenza A viruses, 2009 pandemic H1N1 viruses and emerging highly pathogenic H5N1 virus infection in human beings. The virus fitness of NA inhibitor-resistant viruses of different subtypes in comparison to their respective wild-type viruses in experimental models is also discussed, especially with a focus on the virus transmissibility, one of most clinical relevant properties of influenza A viruses.

\section{NA Inhibitor Resistance in Human Seasonal Influenza A Viruses}

\section{Drug-selected resistance in human seasonal H1N1 and H3N2 influenza A}

viruses. After the NA inhibitors were approved for clinical use in 1999, the resistance to NA inhibitors emerged in clinical isolates during the NA inhibitor treatment in patients infected with seasonal H1N1 or H3N2 influenza viruses (41). The isolation of the resistant variants was associated with NA inhibitor treatment; as a result, they are referred as drug-selected resistance. In the clinical-derived resistant variants, NA inhibitor resistance associated mutations are found to be subtype-specific and drug-specific $(32 ; 42)$, either catalytic residues or the framework residues at the active site. It has been systematically summarized about the resistance-associated mutations found in the human seasonal influenza viruses of different type/subtype, and the susceptibility change to the three NA inhibitors caused by these mutations $(32 ; 42)$. With a focus on clinical isolates only, concisely, the catalytic residue R292K and framework residue E119V mutations 
predominate in resistant $\mathrm{H} 3 \mathrm{~N} 2$ viruses; I222V mutation was reported occasionally in $\mathrm{H} 3 \mathrm{~N} 2$ viruses and N294S mutation was reported in one case of $\mathrm{H} 3 \mathrm{~N} 2$ virus infection, whereas the framework $\mathrm{H} 274 \mathrm{Y} / \mathrm{H} 275 \mathrm{Y}$ (N2/N1 numbering; the actual numbering in the respective subtype were used afterwards) mutation is the most frequent resistance marker in $\mathrm{H} 1 \mathrm{~N} 1$ viruses. At the molecular function level, R292 is part of catalytic residues of enzyme active site responsible for binding to sialic acid and changing its conformational shape during reaction $(43 ; 44)$. The R292K mutation blocked the binding the NA inhibitors or sialic acid, consequently causing crossing-resistance to all three NA inhibitors. E119V mutations at the framework may permit a water molecule binding into the space of the residue Valine (V), which interfere with binding of oseltamivir but not zanamivir or sialic acid to the active site (45), therefore, E119V only results in resistance to oseltamivir, but not to the other two NA inhibitors. The H275Y mutation at the framework does not directly affect the enzyme catalytic sites, but it prevents the reorientation of E277 residue, which is required to accommodate oseltamivir to bind into the active site (43-45). Thus, the H275Y mutation only induces resistance to oseltamivir, not to other two NA inhibitors. It has been proposed that the R292K and N294S mutation may also prevent the reorientation of the E277 residue during the drug molecule binding $(44 ; 45)$.

Serial passages of influenza A viruses in the presence of the NA inhibitors in cells or generation of reverse genetic viruses with the mutation at conservative amino acid residues in NA active site have created more NA inhibitors-resistant variants in vitro, with different levels of resistance to NA inhibitors (32;42). The resistance associated NA mutations identified in vitro is a growing list, which includes but is not limited to D151E, R152K, R371K, R118K, R224K, E227D, E276D, E119G/D/E etc mutations (N2 numbering) $(32 ; 42)$. These mutations have not been found in the clinical isolates yet, perhaps due to their extremely reduced virus replication and infectivity, but they are of interest to understand the catalytic mechanism of NA active site and of implication for surveillance monitoring of NA inhibitor susceptibility.

The detection rates of resistance from patients receiving NA inhibitor treatment in different clinical trials and clinical observation studies varied, depending on the treated subject and influenza virus type/subtype. For seasonal influenza A viruses, the incidence generally remained at low level till 2007. In early clinical trials of oseltamivir use for treatment of seasonal influenza, the emergence rate of resistance was about $<1 \%$ in adults and $4 \sim 8 \%$ in children during the treatment, where the resistance was associated with $\mathrm{H} 275 \mathrm{Y}$ mutation in H1N1 viruses, E119V and R292K mutation in H3N2 viruses $(41 ; 46)$. In later clinical observations, increased frequency of resistant variants detection $(16 \%$ and $27 \%$ ) in drug-treated children was observed, and the resistance was also solely linked to the $\mathrm{H} 275 \mathrm{Y}$ mutation in H1N1 viruses $(41 ; 47)$.

The surveillance studies of NA inhibitors susceptibility of community isolates, which had no identified history of antiviral use in association with collection of the original clinical specimens, are informative to provide the prevalence of resistance in circulating viruses. Two early surveillance studies $(46 ; 48)$ conducted during the first three years of NA inhibitor use (2000 2003) tested thousands of influenza virus isolates 
collected worldwide, and found no isolate or only 8 isolates resistant to oseltamivir respectively $(0 \%$ or $0.33 \%$ resistance rate respectively), which suggesting extremely low prevalence of resistant viruses by then. More surveillance studies $(32 ; 49 ; 50)$ conducted between 2003 2007 by different groups tested more influenza virus samples either collected globally or regionally from different countries including the ones of greatest oseltamivir use, constantly showed very low resistance rates $(0.5 \% \sim 1.7 \%)$ in characterized samples, suggesting that the very low prevalence of resistance in circulating viruses and no potentially transmissible resistance. However, the situation changed dramatically during the 2007-2009 season, when seasonal H1N1 viruses with common H275Y NA mutation were detected with high prevalence world widely under the context of absence of oseltamivir use (45). In differentiation with the drug-selected resistance above, this special phenomenon in the history of NA inhibitor resistance monitoring is referred as naturally occurring resistance and is further discussed in the section below.

The virus fitness of NA inhibitor-resistant seasonal influenza viruses including infectivity and transmissibility compared with respective to wild-type viruses have been studied in experimental animal models (51-53). As regards to H1N1 viruses, in an earlier study, the $\mathrm{H} 275 \mathrm{Y}$ mutant viruses exhibited severely compromised replication and virulence both in vitro and in ferrets compared to the wild-type viruses $(54 ; 55)$. The transmissibility of $\mathrm{H} 275 \mathrm{Y}$ mutant viruses were firstly investigated in a ferret direct contact model, and the direct-contact transmission of a H275Y mutant of A/New Caledonia/20/99-like (H1N1) virus required a higher dose of inoculums than transmission of the wild-type viruses, and it occurred more slowly (53). Regarding to H3N2 viruses, an R292K mutant of A/Sydney/5/97-like H3N2 virus exhibited severely compromised infectivity and replication ability in ferret in comparison with the wild-type virus (55), and was not transmitted by direct contact among ferrets under the same conditions where the wild-type virus was transmitted (52). In a subsequent study, under similar conditions, an E119V NA mutant of A/Wuhan/359/95-like (H3N2) virus by ferret was transmitted by direct contact among ferrets but required a higher dose of inoculums than the wild-type virus, and the transmission occurred more slowly than the wild-type virus (53). In a different animal model, recombinant H3N2 influenza viruses carrying the E119V NA mutation or the double mutations E119V and I222V were transmitted efficiently among guinea pigs by direct contact but not by aerosol in comparison with the wild-type virus (51).

These earlier studies differed in the virus subtypes studied (H1N1, H3N2), the NA mutations involved (H275Y, R292K, E119V or I222V), the animal model used (ferret or guinea pig), and the transmission routes studied (direct contact and respiratory droplets), but they commonly and consistently showed the virus fitness including infectivity and transmissibility of the NA inhibitor-resistant viruses carrying different NA mutations was lower sometimes even severely lower than the respective sensitive wild-type viruses, therefore, the NA inhibitor-resistant viruses were thought unlikely to be of clinical consequence. However, the later studies of virus fitness of the naturally resistant H1N1 viruses showed very contrasting results, which were discussed in below section. 
Naturally-occurring resistance in human seasonal H1N1 influenza A viruses. An exceptional phenomenon in the NA inhibitor resistance detection called naturally-occurring oseltamivir-resistance was observed in the seasonal H1N1 influenza viruses during two influenza seasons between 2008 2009 (45). During these seasons, the H1N1 influenza viruses with the common oseltamivir-resistance NA mutation H274Y (274 in N2 numbering, 275 in N1 numbering) were detected with high prevalence world wide under the context of absence of oseltamivir use $(31 ; 45 ; 56)$. This particular phenomenon was a striking change compared with the situation during the years prior to 2007, when the resistance incidence were usually connected with drug use and the detection rates were rather low. In differentiation with the drug-selected resistance, this special phenomenon is referred as naturally-occurring oseltamivir-resistance caused by the naturally resistant $\mathrm{H} 1 \mathrm{~N} 1$ viruses carrying the H275Y NA mutation in circulation.

The naturally-occurring oseltamivir-resistance was firstly observed in Europe during the 2007-2008 season (57). In January 2008, Norway was the first to report high prevalence $(68 \%)$ of resistant $\mathrm{H} 1 \mathrm{~N} 1$ viruses carrying the $\mathrm{H} 275 \mathrm{Y}$ mutation in circulation (58). In early 2008, an average prevalence of resistant $\mathrm{H} 1 \mathrm{~N} 1$ viruses reached to $56 \%$ across the Europe (57). The resistant H1N1 viruses continue to spread to the southern hemisphere in following influenza season, such as Oceania, South Africa and South East Asia etc regions (59). By mid 2008, the southern hemisphere influenza season marked by a dramatic increase of the resistance rates from $<1 \%$ to $>90 \%$ in less than a year (59). In the following 2008 2009 influenza season, almost 100\% of tested circulating H1N1 viruses were resistant $\mathrm{H} 275 \mathrm{Y}$ mutants in North America and other regions of the world (60). Clearly, the naturally resistant H1N1 variants were becoming the dominant circulating strains until the swine-origin pandemic $2009 \mathrm{H} 1 \mathrm{~N} 1$ virus emerged.

The rapid global surge of naturally resistant H1N1 viruses suggests that these $\mathrm{H} 275 \mathrm{Y}$ mutant viruses owned higher virus fitness including transmissibility than their precedent wild-type viruses, although the previous epidemiologic and experimental data suggested otherwise. In view of this controversy, one study compared the effects of the $\mathrm{H} 275 \mathrm{Y}$ mutation on the replication and virulence of old and recent influenza H1N1 viruses (61). The results showed that the $\mathrm{H} 275 \mathrm{Y}$ mutation caused impaired virus growth in cells only in the old A/WSN/33 virus but not in the recent A/Brisbane/59/07-like virus; the virulence of the recent $\mathrm{A} / \mathrm{Brisbane} / 59 / 07-$ like $\mathrm{H} 275 \mathrm{Y}$ variant virus was not reduced in ferrets compared with the wild-type virus, consistent with its global dissemination.

It has remained a puzzling question for a long time about the underlying mechanism of the optimal fitness of these naturally resistant $\mathrm{H} 275 \mathrm{Y}$ mutant viruses, because the previous epidemiologic and experimental data suggested otherwise about fitness of the H275Y mutant H1N1 viruses. Surveillance studies provided informative clues for this question by examining the genetic changes in the H275Y mutant H1N1 viruses. The phylogenetic analyses by different groups using various circulating H1N1 viruses collected from respective regions showed two common features of these viruses (62-66). First, the NA and HA genes of seasonal H1N1 viruses exhibited same cluster patterns, which were classified as Clade 1, Clade 2A, 2B, 2C, represented by H1N1 vaccine strains New Caledonia/20/1999 virus, Solomon Island/23/2006 virus, 
Brisbane/59/2007 virus respectively (though no vaccine strain representing the Clade 2C); second, the vast majority of the naturally resistant viruses belonged to the Clade 2B in the phylogeny. Some studied further looked the genetic makeup of entire genome of the sensitive and resistant viruses and found that certain NA and HA amino acid substitutions and some intra-subtype reassortment events were associated with the naturally resistant viruses in phylogeny $(65 ; 66)$. These findings of genetic features in the naturally resistant viruses, especially their NA genes clustering together in the Clade 2B are enlightening to further understand their underlying molecular mechanisms.

Two pioneering studies discovered different molecular phenotypes of the naturally resistant $\mathrm{H} 1 \mathrm{~N} 1$ viruses from different angles $(67 ; 68)$, and significantly advanced our understanding of the molecular mechanism of the naturally resistant H1N1 viruses. The visionary work by M.A Rameix-Welti et al (68), for the first time, revealed the distinct levels of NA enzyme affinity and distinct cluster patterns of the HA and NA genes among the old and newer isolates of seasonal H1N1 viruses. This study also showed that all the tested naturally resistant $\mathrm{H} 275 \mathrm{Y}$ mutant viruses belonged to the high NA affinity group which genetically clustered together in the same group. This particular cluster was recognized as Clade 2B later and was consistently showed as a common feature for the naturally resistant H1N1 viruses characterized in different studies (62-66). Further studies have associated the high enzyme affinity in some viruses to certain NA amino acid substitutions $(63 ; 69)$ and one study confirmed the role of D344N substitution in increasing enzyme affinity (70). Another pioneering work by Bloom J. D et al (67), for the first time discovered that surface accumulation of a NA protein was reduced by the H275Y mutation and this defect could be counteracted by two permissive mutations Q222R and V234M which appeared prior to the H275Y mutation in the H1N1 virus evolution. This study is of significance to be the first to move the understanding of the effects of the $\mathrm{H} 275 \mathrm{Y}$ mutation to protein expression level and firstly proposed the concept of "permissive" mutations for the H275Y mutation from the perspective of gene evolution. Following this findings, a recent study confirmed that the substitutions of the identified "permissive" residues into the "unpermissive" residues in the NA gene of a recent Clade2B virus compromised the corresponding $\mathrm{H} 275 \mathrm{Y}$ mutant virus in vitro and in vivo (71).

It remains an uncertain question where these naturally resistant H1N1 influenza viruses originated in the first place. As the H275Y NA mutation was not detected in influenza viruses in nature before the oseltamivir was approved for use at 1999, most likely, the progenitor $\mathrm{H} 275 \mathrm{Y}$ mutant virus of the naturally resistant viruses still firstly emerged from the oseltamivir-treated patient under drug pressure, and the $\mathrm{H} 275 \mathrm{Y}$ mutation was fixed into the ensuing virus population as a results of the identified permissive mutations and/or other unidentified mechanisms. The viruses carrying the $\mathrm{H} 275 \mathrm{Y}$ mutation were further transmitted and spread, eventually becoming the naturally resistant $\mathrm{H} 1 \mathrm{~N} 1$ viruses. Another different and intriguing hypothesis about the origination of resistance is that the NA mutations at the enzyme active site could be a byproduct of HA protein avidity change resulted from immune escape of neutralizing antibodies (27). This study showed that HA immune escape of neutralizing antibodies resulted in NA antigenic variation and acquisition of NA mutations including H274N, V118I and 
K253R, one of which altered the virus susceptibility to NA inhibitors. Although it remained uncertain what prompts the selection of those NA mutations, it provides an alternative view of the origination of the drug resistance.

\section{NA Inhibitor Resistance in 2009 Pandemic H1N1 Influenza A Viruses}

A novel swine-origin H1N1 influenza virus emerged in Mexico in April 2009 and rapidly spread worldwide, causing the first influenza pandemic of the 21 st century. Most confirmed human cases of 2009 pandemic H1N1 virus (pH1N1) infection have been uncomplicated and mild, but the increasing number of cases and affected countries, and the sustained human-to-human transmission of the viruses, warranted optimal prevention and treatment measures. All tested the $\mathrm{pH} 1 \mathrm{~N} 1$ viruses carried resistant makers at M2 gene to amantadine $(72 ; 73)$, so NA inhibitors especially oral oseltamivir were the first line of defense before the antigenically matched influenza vaccine became available. As a result, the oseltamivir use reached unprecedented volume during the 2009 pandemic.

The scale and capability of monitoring of $\mathrm{pH} 1 \mathrm{~N} 1$ virus susceptibility to the NA inhibitor by global and national institutions was also unprecedented in the history (73). Since the detection of the $\mathrm{pH} 1 \mathrm{~N} 1$ viruses in April 2009, the first oseltamivir-resistant pH1N1 virus was reported at Denmark at as early as August 2009, which carried the common oseltamivir resistance marker NA H275Y mutation in N1 subtype and was isolated from a patient receiving oseltamivir prophylaxis (72;74). Subsequently during the pandemic, more sporadic oseltamivir-resistant variants have been isolated from individuals receiving prophylaxis, from immunocompromised patients receiving treatment, and from a few clusters of community isolates with no association to oseltamivir use (73;75-78). As of October 2011, among more than 600 reported oseltamivir-resistant variants (73), most of them are caused by the H275Y NA mutation, with a few exceptions linked to other NA mutations, which are introduced below. The low numbers of detected resistant variants indicate low prevalence of resistance in the circulating $\mathrm{pH} 1 \mathrm{~N} 1$ viruses so far. Different surveillance studies reported from $0 \% \sim 3 \%$ overall detection rate of resistant $\mathrm{pH} 1 \mathrm{~N} 1$ viruses varying from different periods and regions $(73 ; 79 ; 80)$. However, the isolation of community transmitted resistant $\mathrm{pH} 1 \mathrm{~N} 1$ viruses suggests that such viruses keep certain level of local transmissibility thus reinforces the need for continual surveillance.

The $\mathrm{pH} 1 \mathrm{~N} 1$ viruses have the potential to become multi-NA inhibitor-resistant. In the clinical isolates of the $\mathrm{pH} 1 \mathrm{~N} 1$ viruses, apart from than the common $\mathrm{H} 275 \mathrm{Y}$ mutation in the N1 subtype, several other NA mutations were firstly found in the N1 subtype of NA genes which induce different level of resistance to NA inhibitors $(73 ; 77 ; 81 ; 82)$. The NA I223R/I222R (N1/N2 numbering) mutation was identified in the clinical isolates of a patient receiving oseltamivir treatment, and this mutation alone can reduce the $\mathrm{pH} 1 \mathrm{~N} 1$ virus susceptibility to all three NA inhibitors at a small degree (82). The I223R plus $\mathrm{H} 275 \mathrm{Y}$ double mutations future enhanced the resistant level to oseltamivir. Another form of mutation $\mathrm{I} 223 \mathrm{~V}$ in the presence of $\mathrm{H} 275 \mathrm{Y}$ mutation also has been identified in clinical isolates (77), which further enhanced the resistance to oseltamivir and peramivir, but did 
not induced significant resistance to zanamivir (83). The NA S247N mutation was founded at high frequency $(10 \sim 30 \%)$ in several clusters of community isolates of $\mathrm{pH} 1 \mathrm{~N} 1$ viruses (81). The S247N alone induced mildly reduced susceptibility to oseltamivir and zanamivir, not to peramivir, and the S247N plus H275Y double mutations significantly further enhanced the resistance level to oseltamivir, which have been found in clinical isolates (81).

In view of the precedent example of the naturally resistant seasonal H1N1 viruses, understanding the virus fitness of $\mathrm{H} 275 \mathrm{Y}$ mutant resistant $\mathrm{pH} 1 \mathrm{~N} 1$ viruses in laboratory based experiments were of special urgency under the pandemic context. Diverse isolates of $\mathrm{H} 275 \mathrm{Y}$ mutant $\mathrm{pH} 1 \mathrm{~N} 1$ viruses were subjected to extensive characterization and assessment of infectivity and transmissibility in different animal models by individual groups (84-88). The results across different studies reached some agreement on certain conclusion that the $\mathrm{H} 275 \mathrm{Y}$ mutant $\mathrm{pH} 1 \mathrm{~N} 1$ viruses had replication capability, direct-contact transmissibility and overall virulence in animals similar or equivalent to those of the wild-type viruses. However, these studies showed the biggest variation on the transmissibility by respiratory droplet or aerosol route. Compared with their respective wild-type viruses, two studies showed greatly reduced respiratory-droplet transmissibility of two different $\mathrm{H} 275 \mathrm{Y}$ resistant $\mathrm{pH} 1 \mathrm{~N} 1$ isolates in ferrets $(84 ; 86)$; one study exhibited delayed respiratory-droplet transmissibility in one tested $\mathrm{pH} 1 \mathrm{~N} 1$ isolates but essentially the same transmissibility in another isolate in ferrets (87); one study observed the equivalent respiratory-droplet transmissibility of two reverse-genetics derived $\mathrm{H} 275 \mathrm{Y}$ mutant $\mathrm{pH} 1 \mathrm{~N} 1$ viruses in both ferrets and guinea pigs (88). Nevertheless, these different results might be partly due to the various sources of $\mathrm{pH} 1 \mathrm{~N} 1$ viruses with subtle different genetic backgrounds and different experimental settings. The fact that the oseltamivir-resistant H1N1/2009 viruses remained certain level of transmissibility underlines the necessity of continuous monitoring of drug resistance of circulating pH1N1 viruses.

The clinical-derived NA inhibitor resistant $\mathrm{pH} 1 \mathrm{~N} 1$ viruses carrying the new resistance mutations also raised the concern about their virus fitness. Some of the resistant variants have been assessed for their fitness especially transmissibility relative to wild-type viruses in experiments. Regarding to NA 223 mutation, one study showed that an I223R mutant virus isolate has comparable replication ability and transmissibility but lower pathogenicity in ferrets than the wild-type virus (89). Another study observed that the virus infectivity and transmissibility in ferrets were comparable among the wild-type, the H275Y only mutant and the I223V plus H275Y mutant $\mathrm{pH} 1 \mathrm{~N} 1$ viruses generated by reverse genetics techniques (90). As regards to the NA S247N mutation, in a study using reverse-genetics pH1N1 viruses in guinea pigs (91), the S247N mutation only virus showed reduced respiratory-droplet transmissibility and the S247N plus H275Y double mutation exhibited even further reduced transmissibility compared to the wild-type virus, however, when the reverse-genetics $\mathrm{pH} 1 \mathrm{~N} 1$ virus were rescued from another NA genetic background of $\mathrm{pH} 1 \mathrm{~N} 1$ virus, the S247N mutation only and S247N plus H275Y double mutations caused the same reduced transmissibility compared to the wild-type virus. These results suggest that the diverse NA genetic background can cause variation of the virus fitness especially with the presence of the resistance-associated NA mutations. 


\section{NA Inhibitor Resistance in Human Infection of Highly Pathogenic H5N1 Influenza A Viruses}

Highly pathogenic avian $\mathrm{H} 5 \mathrm{~N} 1$ viruses have special clinical relevance to human beings. Since the first human cases in 1997 in Hong Kong (92), sporadic human infection with highly pathogenic avian H5N1 influenza virus has been reported across Asia, Europe, Africa and the Middle East (9). Human infection of highly pathogenic H5N1 virus exhibited severe pneumonia or acute respiratory distress syndrome with a mortality rate higher than $60 \%(9 ; 93)$. In view of that seasonal influenza vaccine does not have effective cross reactivity again the $\mathrm{H} 5 \mathrm{~N} 1$ influenza viruses, the antiviral drugs especially NA inhibitors are standing at the first line of defense of the H5N1 virus infection.

The NA inhibitor resistance in $\mathrm{H} 5 \mathrm{~N} 1$ viruses is a serious concern for public health, thus the susceptibility of H5N1 virus has been evaluated and human infection of $\mathrm{H} 5 \mathrm{~N} 1$ has been closely monitored. Phenotypic resistance test using H5N1 viruses from south-east Asia showed that the viruses were generally sensitive to NA inhibitors but with variability, depending on virus clades and isolation time (9;25). In human infection of highly pathogenic $\mathrm{H} 5 \mathrm{~N} 1$ viruses, oseltamivir-resistant variants have been isolated from patients receiving oseltamivir treatment or prophylaxis and resistance were associated with two mutations H275Y or N294S at NA framework (9;94). Likewise in human N1 subtype viruses, the $\mathrm{H} 275 \mathrm{Y}$ NA mutation in $\mathrm{H} 5 \mathrm{~N} 1$ viruses induced resistance to oseltamivir and reduced susceptibility to peramivir but not to zanamiivir. The N294S NA mutation was identified in one case of H3N2 human influenza virus previously (41), and was only found in clinical isolates of H5N1viruses of N1 subtype so far (9;95), which induced moderate resistance to oseltamivir. By far, the $\mathrm{H} 275 \mathrm{Y}$ vairant were isolated from 3 cases during the N294S variant were isolated from 2 cases in Egypt (9;96;97). One highly pathogenic $\mathrm{H} 5 \mathrm{~N} 1$ virus isolated from a patient treated with oseltamivir had a mixed oseltamivir-sensitive and -resistant population, and the later were identified as either H274Y or N294S NA mutations by plaque purification (97).

The infection of highly pathogenic $\mathrm{H} 5 \mathrm{~N} 1$ viruses in animal models exhibited some special features different from human seasonal influenza viruses, such as extremely high viral loads, systematic spread nature etc, high lethality etc (98). Especially, the highly pathogenic $\mathrm{H} 5 \mathrm{~N} 1$ viruses can effectively infect mouse and cause lethality without the need of prior adaptation like the human seasonal influenza viruses, making the mouse model for useful evaluation of the virus fitness of $\mathrm{H} 5 \mathrm{~N} 1$ viruses. In both mouse and ferret model, the reverse-genetics variants of a highly virulent strain A/Vietnam/1203/04 H5N1 virus carrying either the $\mathrm{H} 275 \mathrm{Y}$ or N294S NA mutation retained their replication efficiency and pathogenicity in vitro and in vivo similar to the wild-type H5N1 virus (99). Also tested in both mouse and ferret models, the clinical-derived variants of A/Hanoi/30408/2005 H5N1 virus carrying either the H275Y or N294S NA mutation were attenuated in terms of pathogencity compared to the wild-type virus, with a bigger degree of attenuation in the H275Y mutant than in the N294S mutant $(97 ; 100)$. It is worth to note that both the H5N1 viruses both belong to HA clade 1 of $\mathrm{H} 5 \mathrm{~N} 1$ virus phylogeny, but A/Hanoi/30408/2005 overall was less virulent than the A/Vietnam/1203/04 virus in tested animal models $(99 ; 100)$. In a virus growth compitition 
experiment for direct comparasion of virus fitness in ferrets (101), as for a clade 2.2 $\mathrm{H} 5 \mathrm{~N} 1$ virus, the virus fitness of reverse-gnetics H275Y mutant of A/Turkey/15/2006-like $\mathrm{H} 5 \mathrm{~N} 1$ virus was less than the wild-type virus, but regarding to a clade $1 \mathrm{H} 5 \mathrm{~N} 1$ virus, the virus fitness of the reverse-genetics H275Y mutant of A/Vietnam/1203/04 H5N1 virus was equivalent to the wild-type virus, suggesting the virus fitness change of the resistant $\mathrm{H} 5 \mathrm{~N} 1$ viruses were associated with the virus genetic background, other than the resistance markers.

\section{Concluding Remarks}

The development of NA inhibitor resistance is a huge drawback to the control of influenza infection by antiviral drugs. The experimental studies of the NA inhibitor-resistant influenza viruses of different type/subtypes have significantly advanced our understanding of the molecular mechanism of the resistance, change of virus fitness in the resistant viruses. These studies have exerted significant implications on surveillance monitoring of susceptibility of circulating influenza viruses to NA inhibitors. On the other hand, some different studies of fitness of the resistant virus of a same resistance marker reached disparate conclusions towards their clinical relevance. The reasons for such discrepancy could be simply from the virus variants used, or the different animal models tested or the diverse experiment setting used. The different genetic backgrounds of the viruses could be subtle difference in NA gene contexts such as in the naturally resistant H1N1 viruses vs the drug-induced resistant H1N1 viruses, or could be in the genome such as in the highly pathogenic H5N1 viruses of different clades. The further advancement of understanding molecular mechanism of fitness of NA inhibitor-resistant viruses necessitates combination of multiple parameters at different levels from viral genes to viral proteins to viruses. The risk of emergence of NA inhibitor-resistant influenza viruses with undiminished fitness should be an alert for surveillance monitoring and experimental studies of circulating influenza viruses.

Overall, NA proteins play an essential role in influenza A virus infection in hosts, and the use of NA inhibitors for treatment of influenza virus infection has induced resistant viruses with different NA mutations. Consequently, how the resistance associated mutations affect NA function and fitness of the mutant influenza viruses remain a big question and are subject for further investigation. In the following two chapters, two studies investigated the impacts of an oseltamivir resistance-associated mutation (NA H275Y) in the 2009 pandemic H1N1 virus or the seasonal H1N1 virus background on the virus in vitro and in vivo fitness including transmissibility. These studies aim to evaluate and understand resistant-virus fitness by integrating multiple parameters at different levels from genes to proteins to viruses. The related backgrounds, specific questions and aims of each study are introduced separated in each chapter. 


\section{CHAPTER 2. THE VIRUS FITNESS AND TRANSMISSIBLITY OF AN OSELTAMIVIR-RESISTANT 2009 PANDEMIC H1N1 INFLUENZA A VIRUS*}

\section{Introduction}

A novel swine-origin H1N1 influenza virus emerged in Mexico in April 2009 and rapidly spread worldwide, causing the first influenza pandemic of the 21 st century $(6 ; 7)$. Most confirmed human cases of H1N1/2009 influenza have been uncomplicated and mild (102), but the increasing number of cases and affected countries warrant optimal prevention and treatment measures. At present, two classes of antiviral drugs are approved for specific management of influenza: M2-ion channel blockers (amantadine and rimantadine) and neuraminidase (NA) inhibitors (zanamivir and oseltamivir). However, variants resistant to both classes of drugs have emerged. During the 2007-2008 season, most circulating seasonal H3N2 influenza viruses, and $\mathrm{H} 1 \mathrm{~N} 1$ viruses in certain geographic areas, were reportedly resistant to M2-blockers $(103 ; 104)$; today, almost all of the pandemic H1N1/2009 viruses tested are resistant to M2-blockers (72). Therefore, only the NA inhibitors are currently recommended for treatment of influenza (105).

The NA-inhibitor resistance-associated mutations in influenza viruses are drug-specific and NA subtype-specific (106). Until 2007, the clinical data indicated only sporadic, rare emergence of oseltamivir resistance under drug selection pressure $(<1 \%$ in adults and $4 \%-8 \%$ in children) $(41 ; 107 ; 108)$. Later reports observed increased frequency of oseltamivir-resistant variants $(16 \%$ and $27 \%)$ in drug-treated children $(47 ; 108)$. The situation changed dramatically during the 2007-2008 season, when seasonal H1N1 influenza viruses with the common oseltamivir-resistance NA H275Y mutation (275 in N1 numbering, 274 in N2 numbering) became widespread in first the northern (109) and then the southern (59) hemispheres. It remains uncertain where these naturally resistant H1N1 influenza viruses originated and how they acquired optimal fitness and transmissibility, but the resistant variants were clearly becoming the dominant strain at the time the swine-origin pandemic H1N1/2009 virus emerged $(45 ; 56 ; 60)$. During the H1N1/2009 influenza pandemic, to date, almost all tested viruses have remained susceptible to oseltamivir and zanamivir (72), but oseltamivir-resistant variants with $\mathrm{H} 275 \mathrm{Y}$ NA mutation have been isolated from individuals receiving prophylaxis $(77 ; 110)$ and from immunocompromised patients (76) under drug selection pressure. Oseltamivir-resistant variants also have been isolated from untreated patients $(111 ; 112)$ and from a few community clusters $(75 ; 113 ; 114)$, including two suspected cases of nosocomial transmission among immunocompromised patients $(75 ; 114)$, although it is uncertain whether the mutants came from secondary transmission or arose spontaneously. The

* Chapter 2 adapted with permission. Duan,S., Boltz,D.A., Seiler,P., Li,J., Bragstad,K., Nielsen,L.P., Webby,R.J., Webster,R.G., and Govorkova,E.A. 2010. Oseltamivir-resistant pandemic H1N1/2009 influenza virus possesses lower transmissibility and fitness in ferrets. PLoS. Pathog. 6:e1001022. 
isolation of resistant $\mathrm{H} 1 \mathrm{~N} 1 / 2009$ viruses with no link to oseltamivir use raised serious concern that these viruses might acquire fitness and spread worldwide, as had oseltamivir-resistant seasonal H1N1 viruses during 2007-2008 season.

The increasing concern about oseltamivir-resistant $\mathrm{H1N1} / 2009$ viruses prompted us to evaluate transmissibility and growth fitness of one oseltamivir-resistant variant. The infectivity and transmissibility (and thus the clinical relevance) of several NA inhibitor-resistant influenza viruses have previously been studied in experimental animal models $(51 ; 52 ; 115 ; 116)$. These studies differed in the influenza A subtypes studied (H1N1, H3N2, or H5N1), the NA mutations involved (H275Y, R292K, E119V or I222V), the animal model used (ferret or guinea pig), and the transmission routes studied (direct contact and respiratory droplets); in these studies, the transmissibility of most of the NA inhibitor-resistant influenza viruses was to some extent less efficient. Here we characterized in vitro and in a ferret model a pair of pandemic H1N1/2009 clinical isolates. The pandemic A/Denmark/524/09 (A/DM/524/09) and A/Denmark/528/09 (A/DM/528/09) viruses were isolated from a small cluster of patients with H1N1/2009 virus infection. The $\mathrm{A} / \mathrm{DM} / 528 / 09$ virus, carrying the $\mathrm{H} 275 \mathrm{Y}$ NA mutation, was isolated from a patient on oseltamivir prophylaxis, and its ancestor is likely to have been $\mathrm{A} / \mathrm{DM} / 524 / 09$ virus. By recapitulating two natural routes of influenza virus transmission in ferrets, we found that the oseltamivir-resistant virus was less transmissible than its sensitive counterpart through the respiratory droplet route but retained efficient transmission through direct contact.

\section{Results}

\section{Sequencing and Phylogenetic Analysis of NA Genes}

Sequence analysis of the NA genes revealed that $\mathrm{A} / \mathrm{DM} / 524 / 09$ virus encoded a conserved $\mathrm{H}$ residue at amino acid position 275, whereas $\mathrm{A} / \mathrm{DM} / 528 / 09$ virus had an $\mathrm{H} 275 \mathrm{Y}$ amino acid mutation caused by a single T-to-C nucleotide substitution at codon 275 (Table 2-1). Pairwise sequence analysis of the full viral genomes showed that the $\mathrm{A} / \mathrm{DM} / 524 / 09$ and $\mathrm{A} / \mathrm{DM} / 528 / 09$ viruses had no amino acid differences other than the H275Y NA mutation and were a highly matched pair. Sequence analysis and phylogenetic analysis of the two viruses NA and HA genes (data not shown) confirmed that the wild-type $\mathrm{A} / \mathrm{DM} / 524 / 09$ and mutant $\mathrm{A} / \mathrm{DM} / 528 / 09$ viruses belonged to the swine-origin 2009 pandemic virus lineage. The alignment of the NA and HA sequences showed that viruses with $\mathrm{H} 275 \mathrm{Y}$ NA substitution have some amino acid differences from certain wild-type viruses (without H275Y NA mutation), but these differences also were observed in other wild-type viruses. Comparison of the NA and HA amino acid sequences of $\mathrm{A} / \mathrm{DM} / 528 / 09$ virus with sequences of other $24 \mathrm{H} 275 \mathrm{Y}$ mutants and around 2000 wild-type H1N1/2009 viruses available in Gene Bank did not reveal an increased frequency of any specific amino acid mutation(s) shared among the viruses analyzed (data not shown). 
Table 2-1. Neuraminidase enzymatic properties of the H1N1 influenza viruses.

\begin{tabular}{|c|c|c|c|c|c|c|}
\hline \multirow{2}{*}{ H1N1 viruses } & \multicolumn{2}{|c|}{$\begin{array}{l}\text { Sequence at NA } \\
\text { position } 275\end{array}$} & \multicolumn{2}{|c|}{$\begin{array}{l}\text { NA enzyme inhibition } \\
\quad \mathrm{IC}_{\mathbf{5 0}} \pm \mathrm{SD}^{\mathrm{a}}(\mathbf{n M})\end{array}$} & \multicolumn{2}{|c|}{ Enzyme kinetics $^{\mathrm{b}}$} \\
\hline & Nucleotide & Amino acid & $\begin{array}{l}\text { Oseltamivir } \\
\text { carboxylate }\end{array}$ & Zanamivir & $\mathbf{K m}(\mu \mathbf{M})$ & $\begin{array}{c}\text { Vmax } \\
(\mathrm{U} / \mathrm{sec})\end{array}$ \\
\hline \multicolumn{7}{|l|}{ Pair 1 (pandemic) } \\
\hline A/Denmark/524/09 & $\mathrm{CAC}$ & $\mathrm{H}$ & $5.0 \pm 0.8$ & $1.3 \pm 0.15$ & $55.1 \pm 4.2$ & $101.6 \pm 7.9$ \\
\hline A/Denmark/528/09 & TAC & $\mathrm{Y}$ & $972 \pm 283^{*}$ & $1.0 \pm 0.13$ & $80.3 \pm 6.0 *$ & $86.8 \pm 5.6^{*}$ \\
\hline \multicolumn{7}{|l|}{ Pair 2 (seasonal) } \\
\hline A/Brisbane/59/07 & CAT & $\mathrm{H}$ & $6.2 \pm 0.6$ & $1.0 \pm 0.1$ & $23.7 \pm 1.7$ & $66.5 \pm 11.2$ \\
\hline A/New Jersey/15/07 & TAT & Y & $1546 \pm 327 *$ & $1.4 \pm 0.1$ & $35.0 \pm 2.8^{*}$ & $37.4 \pm 13.5^{*}$ \\
\hline
\end{tabular}

${ }^{a}$ The full genomes of both viruses were sequenced; only differences are shown. In order of segments, the GenBank accession numbers are CY043339 - CY043346 for A/DM/524/09 virus and CY043347 - CY043354 for A/DM/528/09 virus genome sequences.

${ }^{\mathrm{b}}$ Mean \pm SD from five independent measurements. Assayed in parallel with reference A/Fukui/08/02 (H3N2) virus. Km and Vmax values were derived from the Michaelis-Menten plot.

$* \mathrm{P}<0.05$ compared to value for respective wild-type virus. 


\section{NA Inhibitor Susceptibility and NA Enzyme Kinetics}

To assess the NA inhibitor susceptibility of the two viruses, we performed NA enzyme inhibition assays with the NA inhibitors oseltamivir carboxylate (active metabolite of oseltamivir) and zanamivir. The wild-type A/DM/524/09 virus was susceptible to oseltamivir carboxylate (mean $\mathrm{IC}_{50}: 5.0 \mathrm{nM}$ ), but the $\mathrm{A} / \mathrm{DM} / 528 / 09$ carrying the $\mathrm{H} 275 \mathrm{Y}$ NA mutation had $\mathrm{IC}_{50}$ values approximately 200 times that of the wild-type virus (Table 2-1). The $\mathrm{IC}_{50}$ of zanamivir was comparable for both viruses and was uniformly low (mean $\mathrm{IC}_{50} \leq 1.3 \mathrm{nM}$ ) (Table 2-1). These results showed that the H275Y NA mutation conferred resistance to oseltamivir carboxylate but did not alter susceptibility to zanamivir.

To understand the impact of the $\mathrm{H} 275 \mathrm{Y}$ mutation on the NA enzymatic properties of the H1N1/2009 viruses, we determined the NA enzyme kinetics of both viruses. Km is an estimate of the dissociation equilibrium for substrate binding to enzyme and the reciprocal of $\mathrm{Km}$ approximates the affinity of substrate binding, while Vmax reflects the enzyme's catalytic activity. The NA of resistant A/DM/528/09 virus had a slightly higher $\mathrm{Km}$ and lower Vmax than the NA of the sensitive A/DM/524/09 virus (Table 2-1). The H275Y NA mutation reduced NA affinity for substrate and NA catalytic activity, although the function of NA was not severely impaired. This finding in the H1N1 pandemic virus is similar to that reported by another group, in which NA enzymatic function was not impaired in some naturally resistant seasonal viruses isolated during the 2007 season (117). Our study is the first to show reduced but not severely impaired NA enzymatic function in a resistant $\mathrm{H} 1 \mathrm{N1} / 2009$ virus with the $\mathrm{H} 275 \mathrm{Y}$ mutation.

\section{Plaque Morphology and Growth Kinetics in Cells}

To determine whether the $\mathrm{H} 275 \mathrm{Y}$ NA mutation affects virus growth in vitro, we characterized virus plaque morphology and growth kinetics in both MDCK and MDCK-SIAT1 cells. The latter have increased surface expression of human-like $\alpha 2,6$-linked terminal sialic acids (118) and may better assess the growth capability of human influenza viruses. In MDCK cells, both pandemic H1N1/2009 viruses formed pinpoint-like $(0.3 \mathrm{~mm}$ ) plaque phenotype (Fig 2-1A), differing significantly from some seasonal H1N1 viruses, such as A/Brisbane/59/2007 (BR/59/07) virus, which formed large plaques $(1.3 \mathrm{~mm})(\mathrm{P}<0.05)$ (data not shown); however, the plaque size did not differ between the oseltamivir-sensitive and -resistant viruses (Fig 2-1A), indicating that the H275Y NA mutation did not alter plaque morphology. In MDCK-SIAT1 cells, both the pandemic viruses and seasonal BR/59/07 (data not shown) formed only pinpoint-like plaques (Fig 2-1B), consistent with a previous report (118) that this cell line did not generate clear plaques for influenza viruses.

To further evaluate the impact of the H275Y NA mutation on virus growth in vitro, we performed single- and multiple-cycle growth studies of both viruses in MDCK and MDCK-SIAT1 cells. In single-cycle growth in the two cell lines, the two viruses reached comparable levels 6 hours post-infection, but the initial growth of the 
A

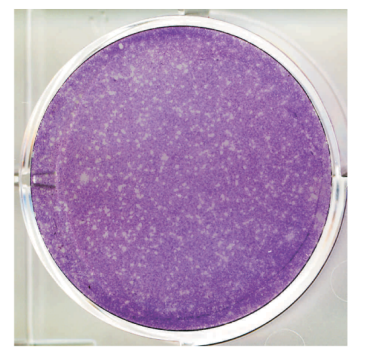

A/DM/524/09

$0.3 \pm 0.04 \mathrm{~mm}$

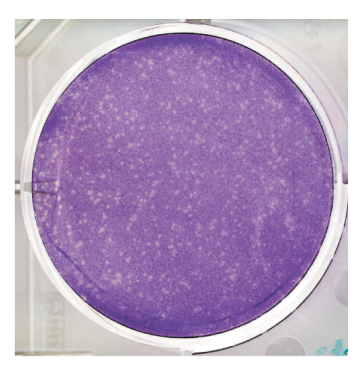

A/DM/528/09

$0.29 \pm 0.06 \mathrm{~mm}$
B
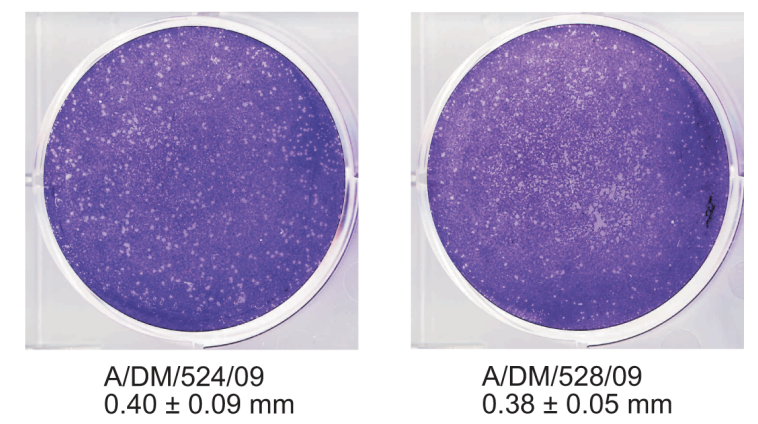

A/DM/528/09

$0.38 \pm 0.05 \mathrm{~mm}$

\section{C}
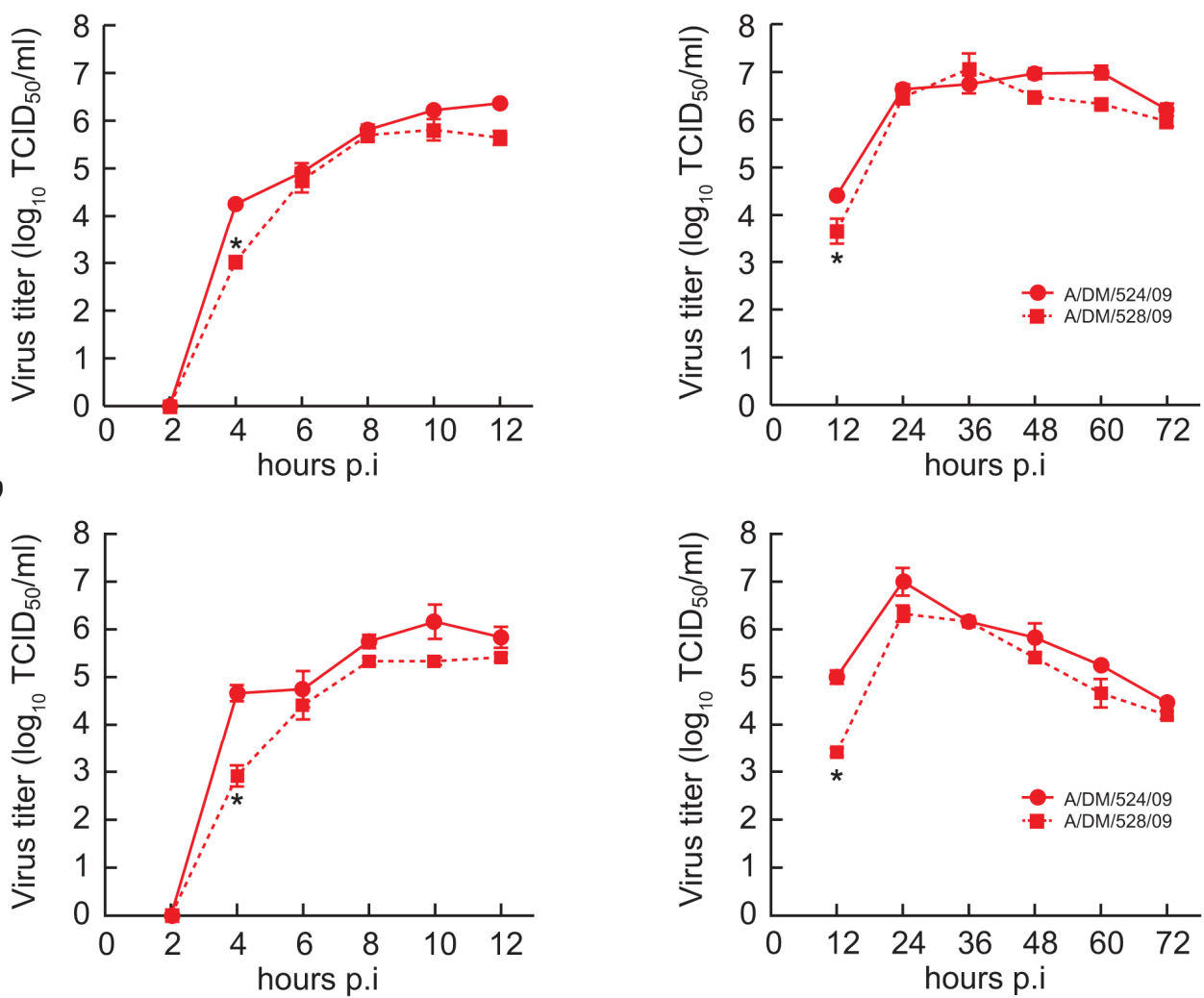

Figure 2-1. Plaque morphology and replication kinetics of two H1N1/2009 influenza viruses in MDCK and MDCK-SIAT1 cells.

The diameters of 20 randomly selected value plaques were measured in MDCK cells (A) and MDCK-SIAT1 cells $(B)$. Values are mean $( \pm \mathrm{SD})$ plaque diameter $(\mathrm{mm})$.

Single-cycle (C, D left panel) and multiple-cycle (C, D right panel) growth curves were obtained by using an MOI of $\sim 2$ and $\sim 0.001 \mathrm{PFU} /$ cell, respectively. Virus in the supernatant was titrated in MDCK or MDCK-SIAT1 cells and expressed as $\log _{10} \mathrm{TCID}_{50} / \mathrm{ml}$ at the indicated time post-infection. Each point represents the mean $\log _{10} \mathrm{TCID}_{50} / \mathrm{ml} \pm \mathrm{SD}$ from three experiments. ${ }^{*} \mathrm{P}<0.05$ compared to value for wild-type viruses. 
oseltamivir-resistant virus was significantly delayed in comparison to its sensitive counterpart $(\mathrm{P}<0.05)$ (Fig 2-1C): at 4 hours post-infection, the yield of resistant viruses was at least $1 \log _{10} \mathrm{TCID}_{50} / \mathrm{ml}$ lower $(\mathrm{P}<0.05)$. Likewise, in multiple-cycle growth, the two viruses reached comparable yields 24 hours post-infection, but the resistant virus showed a significant growth delay during the first 12 hours post-infection $(\mathrm{P}<0.05)$; this delay was more conspicuous in MDCK-SIAT1 cells than in MDCK cells (Fig 2-1D), probably because overexpressed $\alpha 2,6$ receptors on cell surface could better differentiate NA's function in support of viral growth. Therefore, final virus yields of oseltamivir-resistant pandemic virus in the MDCK and MDCK-SIAT1 cells were not altered, but their growth at the initial infection stage was significantly delayed.

\section{Transmissibility among Ferrets via Direct-contact and Respiratory-droplets Routes}

The transmissibility of pandemic H1N1/2009 viruses was studied in a ferret model. Two naïve ferrets were housed at day 2 post-inoculation (p.i.) in the same cage with one inoculated ferret (direct contact), and two naïve ferrets were placed in an adjacent cage separated from the donor's cage by two layers of wire mesh (respiratory droplet exposure). Transmission of $\mathrm{H} 1 \mathrm{~N} 1$ virus was assessed by detection of infection in recipient ferrets (nasal wash titers, clinical signs, and seroconversion). Virus samples in nasal washes at day 4 p.i. or post-contact (p.c.) were sequenced to detect the presence of the $\mathrm{H} 275 \mathrm{Y}$ NA mutation.

The donor ferret inoculated with oseltamivir-sensitive A/DM/524/09 virus shed virus until day 6 p.i. (Fig 2-2A, Table 2-2). Two of 2 direct-contact ferrets and 1 of 2 respiratory droplet-exposed ferrets were infected through virus transmission, as indicated by the virus titers and inflammatory cell counts in their nasal washes (Fig 2-2) and by seroconversion (Table 2-3). Virus shedding and nasal inflammation began earlier in the direct-contact ferrets, suggesting that transmission through respiratory-droplets may have a greater lag time. One respiratory droplet-exposed ferret showed no detectable virus shedding or inflammation, but its post-contact serum had a positive HI titer (320). Although seroconversion indicated infection in this ferret, the time of infection could not be determined and therefore we could not attribute the infection to direct contact with the co-caged ferret versus respiratory droplet transmission from the adjacent cage.

The donor ferret inoculated with oseltamivir-resistant A/DM/528/09 virus shed virus until day 8 p.i. (Fig 2-2), with a peak virus titer comparable to that of A/DM/524/09 virus (Table 2-2). Two of 2 direct-contact ferrets were infected through transmission (Fig 2-2), but neither respiratory droplet-exposed ferret was infected, as confirmed by the absence of seroconversion (Table 2-3). These results showed that the resistant H275Y mutant $\mathrm{A} / \mathrm{DM} / 528 / 09$ virus was transmitted efficiently only by direct contact. Virus shedding in two direct-contact ferrets was lower and peaked after a longer interval in this group than in the oseltamivir-sensitive A/DM/524/09 group (Fig 2-2A), although the severity and course of disease were similar (Fig 2-2B, Table 2-3). 
A


B
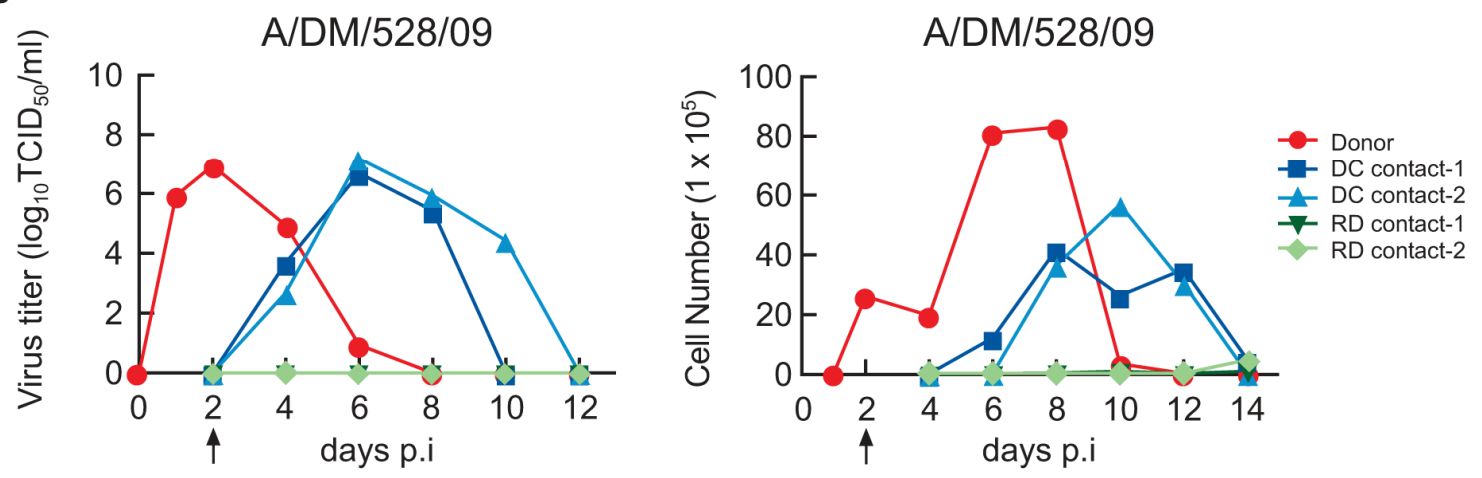

Figure 2-2. Transmissibility of the two H1N1/2009 influenza viruses among ferrets.

The virus titer (A, B left panel) and total number of inflammatory cells (A, B right panel) in the nasal wash samples from each donor ferret, direct-contact (DC contact) ferret, and respiratory droplet-contact ( $\mathrm{RD}$ contact ) ferret. The arrow indicates the first day of exposure of contact ferrets. 
Table 2-2. Clinical signs, virus replication and sero-conversion in inoculated donor ferrets.

\begin{tabular}{|c|c|c|c|c|c|c|}
\hline \multirow{3}{*}{ H1N1 virus } & \multicolumn{6}{|c|}{ Inoculated donor ferrets ${ }^{a}$} \\
\hline & \multicolumn{3}{|c|}{ Clinical signs } & \multicolumn{3}{|c|}{ Virus replication } \\
\hline & $\begin{array}{l}\text { Weight } \\
\operatorname{loss}^{b}(\%)\end{array}$ & $\begin{array}{c}\text { Sneezing } \\
\text { (day of } \\
\text { onset) } \\
\end{array}$ & $\begin{array}{c}\text { Last day } \\
\text { of } \\
\text { inflammation }^{\mathrm{c}} \\
\end{array}$ & $\begin{array}{c}\text { Peak virus } \\
\text { titer }^{\text {d }} \\
\text { (day p.i) }^{d}\end{array}$ & $\begin{array}{c}\text { Last day } \\
\text { of } \\
\text { shedding }\end{array}$ & $\begin{array}{l}\text { Serum } \\
\text { HI titer }\end{array}$ \\
\hline A/Denmark/524/09 & $5.0(2)$ & 3 & 12 & $7.3(2)$ & 6 & 1280 \\
\hline A/Denmark/528/09 & $6.2(2)$ & 7 & 12 & $6.9(2)$ & 8 & 640 \\
\hline Co-inoculation ${ }^{\mathrm{g}}$ & $5.9(4)$ & 7 & 12 & $7.7(2)$ & 6 & 640 \\
\hline
\end{tabular}

${ }^{\mathrm{a}} \mathrm{n}=1$ for each virus group.

${ }^{\mathrm{b}}$ The maximum percent weight loss during the 21 days p.i. Numbers in parentheses indicate the day of maximum weight loss.

${ }^{\mathrm{c}}$ Upper respiratory tract inflammation was defined as a total inflammatory cell count $\geq 10$ times the baseline count.

${ }^{\mathrm{d}}$ Virus titers in nasal washes $\left(\log _{10} \mathrm{TCID}_{50} / \mathrm{ml}\right)$.

e The first day of observation on which virus was not detected.

${ }^{\mathrm{f}}$ Hemagglutination inhibition (HI) antibody titers to homologous virus 21 days p.i.

${ }^{g}$ Co-inoculation of ferret with $\mathrm{A} / \mathrm{DM} / 524 / 09$ and $\mathrm{A} / \mathrm{DM} / 528 / 09$ viruses at 1:1 ratio. 
Table 2-3. Clinical signs, virus replication, and seroconversion in contact ferrets.

\begin{tabular}{|c|c|c|c|c|c|c|c|c|c|c|}
\hline \multirow{3}{*}{ H1N1 virus } & \multicolumn{5}{|c|}{ Direct contact } & \multicolumn{5}{|c|}{ Respiratory droplets } \\
\hline & \multicolumn{2}{|c|}{ Clinical signs } & \multicolumn{3}{|c|}{ Virus detection } & \multicolumn{2}{|c|}{ Clinical signs } & \multicolumn{3}{|c|}{ Virus detection } \\
\hline & $\begin{array}{c}\text { Weight } \\
\operatorname{loss}^{\mathrm{a}}\end{array}$ & Sneezing $^{\mathrm{b}}$ & $\begin{array}{c}\text { Virus } \\
\text { shedding }\end{array}$ & $\begin{array}{c}\text { Last day } \\
\text { of } \\
\text { shedding }\end{array}$ & $\begin{array}{c}\text { Serum } \\
\text { HI } \\
\text { titer }^{\mathrm{e}} \\
\end{array}$ & $\begin{array}{c}\text { Weight } \\
\operatorname{loss}^{\mathrm{a}}\end{array}$ & Sneezing $^{\mathbf{b}}$ & $\begin{array}{c}\text { Virus } \\
\text { shedding }^{c}\end{array}$ & $\begin{array}{c}\text { Last day } \\
\text { of } \\
\text { shedding }\end{array}$ & $\begin{array}{c}\text { Serum } \\
\text { HI } \\
\text { titer }^{\mathrm{e}}\end{array}$ \\
\hline A/Denmark/524/09 & $\begin{array}{c}2 / 2 \\
(3.5)\end{array}$ & $\begin{array}{l}1 / 2 \\
(7)\end{array}$ & $\begin{array}{c}2 / 2 \\
(8.3)\end{array}$ & 8,8 & $\begin{array}{c}1280 \\
640\end{array}$ & $\begin{array}{c}1 / 2 \\
(6.0)\end{array}$ & $\begin{array}{l}1 / 2 \\
(7)\end{array}$ & $\begin{array}{c}1 / 2 \\
(7.2)\end{array}$ & 10 & $\begin{array}{c}1280, \\
320\end{array}$ \\
\hline A/Denmark/528/09 & $\begin{array}{l}2 / 2 \\
(3.3)\end{array}$ & $2 / 2(5,7)$ & $\begin{array}{c}2 / 2 \\
(7.0)\end{array}$ & 8,10 & $\begin{array}{l}1280 \\
1280\end{array}$ & $0 / 2$ & $0 / 2$ & $0 / 2$ & NA & $\begin{array}{l}<10 \\
<10\end{array}$ \\
\hline Co-inoculation ${ }^{\mathrm{f}}$ & $\begin{array}{c}2 / 2 \\
(6.0)\end{array}$ & $\begin{array}{l}1 / 2 \\
(2)\end{array}$ & $\begin{array}{c}2 / 2 \\
(7.1)\end{array}$ & 10,10 & $\begin{array}{l}1280 \\
1280\end{array}$ & NA & NA & NA & NA & NA \\
\hline
\end{tabular}

${ }^{a}$ Number of animals with weight change/total number (maximum percent weight loss during the 21 days p.c.).

${ }^{\mathrm{b}}$ Number of animals sneezing/total number during the 21 days p.c. (day of observed onset).

${ }^{\mathrm{c}}$ Number of virus-shedding animals/total number. Numbers in parentheses indicate mean peak virus titer $\left(\log _{10} \mathrm{TCID} 50 / \mathrm{ml}\right)$ in nasal wash samples).

${ }^{\mathrm{d}}$ The first day of observation on which virus was not detected.

${ }^{\mathrm{e}}$ Hemagglutination inhibition (HI) antibody titers to homologous virus in ferret serum on day 21 p.c.

${ }^{\mathrm{f}}$ Donor ferret was co-inoculated with $\mathrm{A} / \mathrm{DM} / 524 / 09$ and $\mathrm{A} / \mathrm{DM} / 528 / 09$ viruses at a 1:1 ratio. 
We verified the sequence stability of the NA at position 275 in each virus after replication and transmission in ferrets. Direct sequencing of the NA genes from nasal wash samples revealed no sequence change at this position in either virus (data not shown). Therefore, no spontaneous H275Y NA mutation emerged in the wild-type virus and the $\mathrm{H} 275 \mathrm{Y}$ mutation remained stable in the mutants after transmission to a new host.

\section{Co-inoculation with Oseltamivir-sensitive and -resistant H1N1/2009 Viruses}

Because both the oseltamivir-sensitive and the oseltamivir-resistant H1N1/2009 viruses were efficiently transmitted by direct contact, hosts could potentially be exposed to both types of virus. To compare the relative growth capability and transmissibility of the sensitive and resistant $\mathrm{H} 1 \mathrm{~N} 1 / 2009$ viruses within the host, we co-inoculated a ferret with a 1:1 ratio of the sensitive $\mathrm{A} / \mathrm{DM} / 524 / 09$ and resistant $\mathrm{A} / \mathrm{DM} / 528 / 09$ viruses. The pattern of virus shedding and the clinical signs were similar to those in ferrets inoculated with either A/DM/524/09 or A/DM/528/09 virus (Fig 2-3A). By using a relative quantification of single nucleotide polymorphism (SNP) method to detect the NA genotype at codon 275 (CAC or TAC), we found that the virus population in the co-inoculated ferret's nasal washes remained mixed but was predominantly a wild-type (oseltamivir-sensitive) population (Fig 2-3B). The proportion of wild-type virus in the nasal wash increased progressively, from $75 \%$ on day 1 p.i., to almost $100 \%$ on day 6 p.i. (Fig 2-3B). Two of 2 ferrets placed in direct contact with the co-inoculated ferret were infected through transmission (Fig 2-3A). SNP analysis of their nasal wash samples showed only wild-type virus (Fig 2-3B). In summary, the oseltamivir-sensitive $\mathrm{A} / \mathrm{DM} / 524 / 09$ virus possessed greater growth capability in the upper respiratory tract than did resistant $\mathrm{A} / \mathrm{DM} / 528 / 09$ virus and thus had an advantage in direct-contact transmission.

\section{Discussion}

This study is the first, to our knowledge, to demonstrate the inefficient respiratory droplet transmission of an oseltamivir-resistant H275Y mutant of H1N1/2009 in ferrets, which are an established animal model of the pathogenesis and transmission of human influenza viruses. The oseltamivir-resistant mutant virus retained efficient transmission only by direct contact, whereas the oseltamivir-sensitive pandemic virus was efficiently transmitted by both routes. These results show that the transmissibility of the oseltamivir-resistant H1N1/2009 influenza virus had been altered. We suggest that the lower fitness of oseltamivir-resistant variant within the host along with its reduced NA enzyme efficiency and delayed growth of the $\mathrm{H} 275 \mathrm{Y}$ mutant virus in vitro may at least in part explain its impaired transmission among ferrets.

There are limited experimental data about the routes of transmission of oseltamivir-resistant influenza viruses. The two natural routes of influenza virus transmission, direct contact with fomites and respiratory droplets (aerosol and larger droplets (119), are not mutually exclusive. Therefore, the transmissibility of influenza 
A
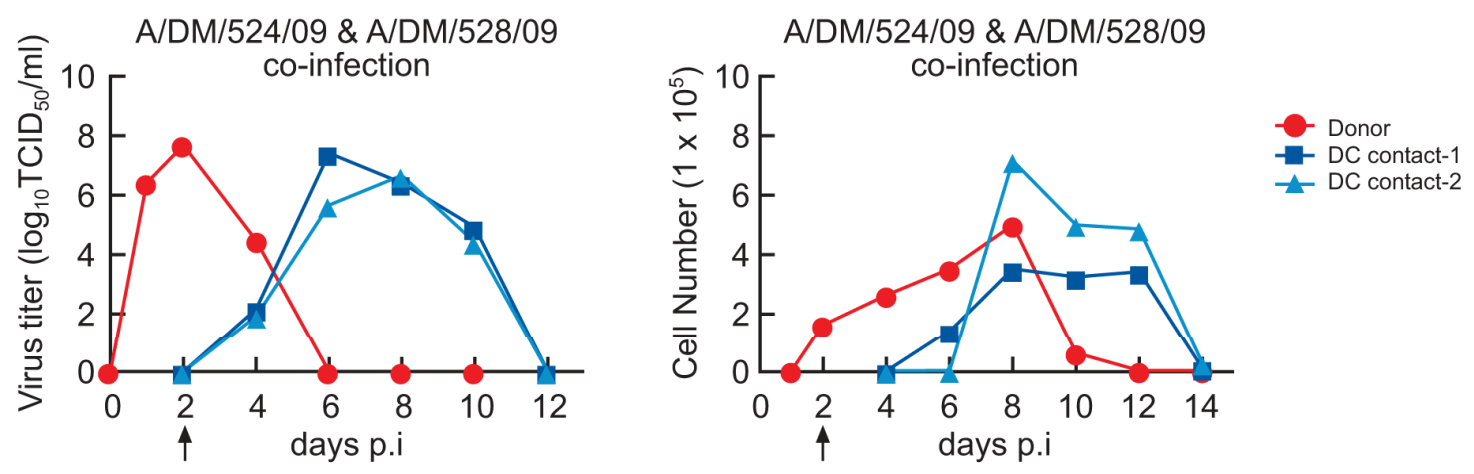

B

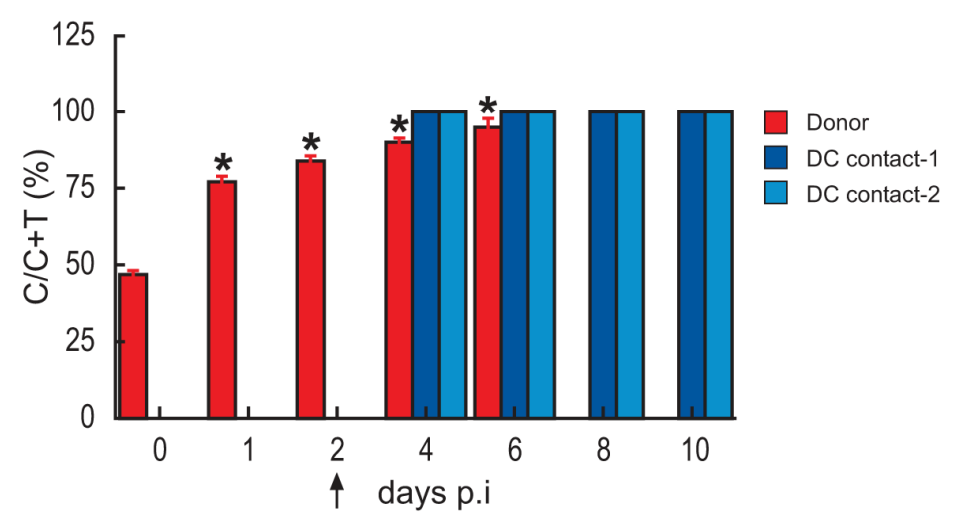

Figure 2-3. Co-infection with oseltamivir-sensitive and -resistant H1N1/2009 influenza viruses.

Virus titers and inflammatory cell counts in the nasal wash specimens of ferrets co-inoculated with oseltamivir-sensitive and -resistant $\mathrm{H} 1 \mathrm{~N} 1 / 2009$ viruses (A). The arrow indicates the first day of exposure of contact ferrets. The proportion of wild-type virus $(\mathrm{C}$ in SNP sequence) in the mixed virus population ( $\mathrm{C}+\mathrm{T}$ in $\mathrm{SNP}$ sequence) in nasal wash samples from the donor ferret and two direct-contact ferrets (B). Values are the mean \pm $\mathrm{SD}$ from three independent measurements. $* \mathrm{P}<0.05$ compared to value for day 0 p.i. 
viruses via both routes must be investigated if the results are to be clinically relevant. In the earliest studies, oseltamivir-resistant H3N2 (R292K NA mutant) and H1N1 (H275Y NA mutant) variants exhibited severely compromised replication and virulence both in vitro and in vivo $(54 ; 55)$ and were therefore thought unlikely to be of clinical consequence. In a subsequent study, an R292K mutant of H3N2 virus was not transmitted by direct contact among ferrets (52). Under similar conditions, the transmission of an E119V mutant of H3N2 virus and an H275Y mutant of A/New Caledonia/20/99-like $\mathrm{H} 1 \mathrm{~N} 1$ virus by direct contact required a higher dose of inoculum than transmission of the wild-type viruses, and it occurred more slowly (120). However, none of these studies assessed both routes of transmission. The only study to date that has evaluated both routes of transmission of oseltamivir-resistant virus showed that recombinant resistant H3N2 viruses with either the E119V or the E119V+I222V NA mutation were transmitted efficiently by direct contact but not by respiratory droplets among guinea pigs (51). Our study is a latest addition to the previous data by comparing a highly matched pair of H1N1/2009 viruses and by assessing the transmissibility of resistant viruses via two routes in ferrets.

The reduced transmissibility of the oseltamivir-resistant $\mathrm{H} 1 \mathrm{~N} 1$ viruses could be explained by a number of factors (121-123). First, host physical exposure to virus is directly affected by the quantity of virus shed into the environment. In our study, inoculated donor ferrets shed comparable quantities of both viruses, which indicated potential comparable environmental contamination in the restricted space of cages; therefore, it is unlikely that transmission was affected by the level of donor viral shedding. Other host variables such as the extent of inflammation could affect the amount and size of upper respiratory secretions thus the release of infectious respiratory droplets. For example, sneezing, a common host symptom believed to mediate viral transmission, was observed only at later stages in the ferret inoculated with resistant virus, when inflammation was more severe but virus shedding had declined greatly. Second, efficient transmission to a naive host requires not only viral exposure but also successful viral invasion, effective replication and simultaneous evasion of the first line of host innate immunity (124). Our results showed a significant initial growth delay in two cell lines of the resistant virus. This growth delay could be caused by delayed release of progeny virions from the host cell surface due to reduced NA enzyme efficiency observed in the resistant virus. Such a delay would not affect the final virus yield in cell lines, but in the respiratory tract of ferrets it could allow the host's first-line innate immune defense (e.g., macrophages or neutrophils) sufficient time to clear the virus. The NA enzyme also facilitates virus binding, entry, and spread within the host by removing terminal sialic-acid residues from mucus and preventing virion self-aggregation (25), and therefore the NA mutation could have affected viral penetration into the host respiratory tract. The slightly reduced (not severely impaired) NA enzyme function and delayed viral growth of the $\mathrm{H} 275 \mathrm{Y}$ mutant may have been more crucial in recipient ferrets that acquired virus from environment via natural routes than in donor ferrets inoculated with a high dose of virus, as we observed delayed viral shedding or inefficient transmission in the recipient ferrets, but not in the inoculated donor ferret. 
Although the transmissibility of the oseltamivir-resistant H1N1/2009 virus was reduced by the $\mathrm{H} 275 \mathrm{Y}$ NA mutation, the severity and course of disease was similar to that caused by oseltamivir-sensitive H1N1/2009 virus in both inoculated and direct-contact ferrets, with no apparent attenuation of clinical signs. In inoculated ferrets, the viruses showed comparable replication in the upper respiratory tract and caused comparable clinical signs, including weight loss and inflammation. However, one caveat to ferret model has been noticed that high inoculation dose may mask the differential viral replication and clinical signs for different viruses (125). In the direct-contact ferrets, which acquired virus though natural routes, the shedding of resistant virus peaked later than the shedding of susceptible virus, but the duration of shedding and the severity of disease was not compromised when compared with sensitive virus. Therefore, the H275Y mutant of pandemic H1N1/2009 virus is likely to be of clinical consequence in humans.

The fitness of a virus describes its relative ability to produce infectious progeny in a host (39). Competitive growth assay by co-infection is a method of evaluating the growth fitness of two viruses $(39 ; 126)$. In the present study, we inoculated a ferret with equal doses of oseltamivir-sensitive and -resistant H1N1/2009 viruses to compare their relative growth fitness within the host. The mixed virus population in the nasal wash was analyzed at different days p.i. to determine which viral genotype predominated. To bypass the time- and labor-intensive process of cloning the desired genes from the mixed populations and choosing an arbitrary number of clones for genotypic analysis, we used a new method, relative quantification of SNP, to determine the ratio of wild-type to mutant populations. This method showed high reproducibility in genotyping HIV protease gene (126). Our study is the first to use this method to genotypically analyze influenza viruses. For the H1N1/2009 influenza viruses, we designed a specific probe to detect the first nucleotide of codon 275 of the NA gene, where a single C-to-T substitution causes an H-to-Y amino acid substitution. Our results showed that the oseltamivir-resistant mutant H1N1/2009 virus possessed less growth fitness than the sensitive H1N1/2009 virus in the ferret upper respiratory tract. At least partly for that reason, only wild-type H1N1/2009 virus was transmitted to the direct-contact ferrets. The competitive transmission advantage of wild-type H1N1/2009 virus should be confirmed by other types of experiments.

In summary, our study determined the comparative transmissibility of a pair of naturally circulating oseltamivir-sensitive and oseltamivir-resistant H1N1/2009 viruses. This information from this study could be useful in assessing the clinical relevance of contemporary pandemic viruses, considering the extensive use of oseltamivir during this pandemic. The H275Y mutant of H1N1/2009 used in this study was the first oseltamivir-resistant $\mathrm{H} 1 \mathrm{~N} 1 / 2009$ isolate from a patient on oseltamivir prophylaxis. As this study was undertaken, additional H275Y mutants of H1N1/2009 viruses have emerged in the absence of oseltamivir use $(114 ; 127)$. The emergence of these viruses should raise concerns as to whether resistant H1N1/2009 viruses will acquire significantly greater fitness and spread worldwide as did the naturally resistant H1N1 viruses during the 2007-2008 season. Further studies of these newly isolated H275Y mutants of H1N1/2009 viruses are warranted to determine whether they have acquired additional changes. 


\section{CHAPTER 3. THE MOLECULAR BASIS OF NATURALLY-OCCURRING OSELTAMIVIR-RESISTANT H1N1 INFLUENZA A VIRUSES}

\section{Introduction}

The neuraminidase (NA) inhibitors (oseltamivir and zanamivir) are among the first line of defense against influenza infection. At present, two classes of antiviral drugs are approved for specific management of influenza: M2-ion channel blockers (amantadine and rimantadine) and neuraminidase (NA) inhibitors (zanamivir and oseltamivir) (28). However, variants resistant to both classes of drugs have emerged. Since 2007 season, most circulating seasonal H3N2 influenza viruses, varying portions of seasonal H1N1 viruses and avian influenza viruses of different subtypes, and almost all tested 2009 pandemic H1N1 viruses were reportedly resistant to M2-blockers $(29 ; 73)$. Therefore, only the NA inhibitors are currently recommended for treatment of influenza.

The resistance to NA inhibitors can significantly limit the infection control options for influenza, so the susceptibility of circulating human influenza viruses to NA inhibitors has been closely monitored. Since the NA inhibitors were approved for clinic use in 1999, the resistant variants had emerged and the resistance detection rates had a radical change over the past decade $(42 ; 45 ; 56)$. Until 2007 , both the clinical observations and surveillance data indicated only sporadic, rare detection rate of resistance to NA inhibitor mainly under drug selection pressure $(41 ; 46 ; 48)$, suggesting extremely low prevalence of resistance among circulating influenza viruses and no evident transmission of resistant viruses. However, the situation changed dramatically during the 2007-2008 season, when seasonal H1N1 influenza viruses carrying the common oseltamivir-resistance NA mutation H275Y (274 in N2 numbering, 275 in N1 numbering) were detected with high prevalence in first the northern $(57 ; 58)$ and then the southern hemispheres (59) with no link to oseltamivir use. In the early 2009 season, almost 100\% of H1N1 virus isolates tested in the USA were found to carry this resistant marker (60). The resistant variants were clearly becoming the dominant strain until the 2009 H1N1 pandemic took over the world $(56 ; 128)$. Naturally-occurring oseltamivir-resistant or naturally resistant $\mathrm{H} 1 \mathrm{~N} 1$ viruses have been used to specifically refer to these seasonal $\mathrm{H} 275 \mathrm{Y}$ mutant resistant $\mathrm{H} 1 \mathrm{~N} 1$ variants which were detected under the contexts of absence of oseltamivir use during the 2007 2009 seasons, in differentiation with the drug-selected viruses isolated from patients treated with NA inhibitors.

It has remained puzzling for a long time how these naturally-resistant $\mathrm{H} 275 \mathrm{Y}$ mutant viruses acquired optimal fitness because the previous experimental and epidemiologic data suggested otherwise $(54 ; 55)$. Two pioneering works on the naturally $\mathrm{H} 275 \mathrm{Y}$ mutant $\mathrm{H} 1 \mathrm{~N} 1$ viruses significantly advanced the understanding to this question from two different angles $(67 ; 68)$. The visionary work by M.A Rameix-Welti et al (68), for the first time, revealed the distinct level of NA enzyme affinity and distinct cluster patterns of NA and HA genes of wild-type and H275Y mutant viruses among the older and new isolates of seasonal H1N1 viruses. In line with this study, subsequently, some phylogeny studies classified the specific cluster patterns of the NA and HA genes as different clades and showed the same trends that vast majority of H275Y mutant H1N1 
viruses, especially the naturally resistant ones, commonly belonged to the Clade 2B (6266). Further studies have associated the higher enzyme affinity in some Clade 2B viruses to certain NA amino acid substitutions $(63 ; 69)$ and one study confirmed the role of NA D344N substitution in NA enzyme affinity (70). Another pioneering work by Bloom J. D et al (67), for the first time discovered that surface accumulation of a NA protein was reduced by the $\mathrm{H} 275 \mathrm{Y}$ mutation and this defect could be corrected by two permissive mutations Q222R and V234M which appeared prior to the H275Y mutation in the NA evolution. This study is of significance to be the first to move the understanding of the defect by the H275Y mutation to NA protein expression level and firstly proposed the concept for the "permissive" mutations for $\mathrm{H} 275 \mathrm{Y}$ mutation from the perspective of gene evolution. Following this findings, a recent study confirmed that the substitutions of the identified "permissive" residues into the "unpermissive" residues in the NA gene of a recent Clade $2 \mathrm{~B}$ virus compromised the $\mathrm{H} 275 \mathrm{Y}$ mutant virus replication in vitro and in vivo (71).

These studies have provided insights to understand the defects in NA protein and virus caused by the H275Y NA mutation and how permissive NA mutations can counteract such defects in NA protein. However, because NA proteins play essential roles in influenza virus normal life cycle, it has been hypothesized that the permissive mutations may not only restore the defect caused by the H275Y mutation, but also may have extended roles beyond what were seen in $\mathrm{H} 275 \mathrm{Y}$ mutant viruses (129). A simple but fundamental question remains open: what were the potential fundamental changes in the of seasonal H1N1 viruses other than $\mathrm{H} 275 \mathrm{Y}$ mutant viruses during the first decade of oseltamivir use. So far no study had been done to learn about the wild-type H1N1 viruses themselves including NA protein function in vitro and virus fitness in vivo.

This present study had started with aims to systematically characterize the virus fitness in vivo and NA phenotypes in vitro of genetically divergent seasonal H1N1 viruses based on the NA phylogeny, also to comprehensively assess which and how the NA amino acid substitution in the NA gene divergence can affect the phenotype. To our surprise, the two "permissive mutations" enabling the H275Y mutation was consistent with our results but two mutations were only half of the growing list of permissive mutations, and even the "permissive mutations" for the $\mathrm{H} 275 \mathrm{Y}$ mutation was only part of the whole story of NA protein evolution. Our results revealed that the natural NA genetic divergence led to significant phenotypic changes of the NA proteins themselves at many aspects, which in return determined the differential effects of the H275Y mutation on the NA proteins. Further study in combination with timeline analysis of the fixation of the divergent NA amino acid residues allow us to proposed a scenario for emergence of the naturally resistant viruses: during the seasonal H1N1 virus evolution, there were subsequent two lineages of H1N1 viruses with NA proteins of distinct attributes at protein level; the $\mathrm{H} 275 \mathrm{Y}$ mutation had detrimental effects on protein expression and function of the old lineage NA proteins, which it only caused a minimal negative effects on the new lineage NA proteins; as results, the H275Y mutant H1N1 viruses of old lineage were extinguished due to severely compromised virus fitness, while the H275Y mutant viruses of new lineage were kept in continuing circulation due to minimally altered virus fitness - the naturally resistant H1N1 viruses. 


\section{Results}

\section{Evolution of NA Genes of Seasonal H1N1 Influenza Viruses}

We firstly assessed the overall genetic difference of the NA genes of seasonal H1N1 viruses by examining their evolution history with a focus on NA H275Y variants isolated from 1999 to 2009 season. The phylogenetic analysis shows that the NA genes between 1999 2009 can be classified into four distinct lineages (Fig 3-1): New Caledonia/20/99-like (NC99-like), Solomon Island/23/06-like (SI06-like), Brisbane/59/07-like (BR07-like), and /Colorado/UR06-0053/2007 (CO07-like), corresponding to the classification of Clade1, Clade2A, Clade2B, Clade2C in previous reports (63-66). The tree also shows that the vast majority of NA H275Y variant isolates accumulate at the BR07-like lineage, and only a few H275Y isolates sparsely scatter at the NC99-like lineage (Fig 3-1). This observation is consistent with the previous surveillance studies of NA inhibitor susceptibility (63-66). To find the essential underlying difference between the two lineages, we compared the NA consensus sequences of both wild-type and $\mathrm{H} 275 \mathrm{Y}$ mutant viruses from the BR07-like and NC99-like lineages. The comparison revealed that apart from the resistance-associated $\mathrm{H} 275 \mathrm{Y}$ mutation, there are only ten amino acid residues substitutions differentiating the NA genotype between the BR07-like and NC99-like lineages (Table 3-1). It is noteworthy that there is one predominant amino acid substitution (D354G) between the NA genes of wild-type and H275Ymutant viruses within the BR07-like lineage, and it is a reversion of the G354D substitution between the NC99-like and BR07-like lineages. We mapped the positions of the ten amino acid residues into a N1 molecule structure (Fig 3-2), and found that none of them are located at the NA enzyme active site. The position 45 and 78 are located at stalk region of NA molecule (not shown in the structure view); the position 222, 234, 249 and 344 are located on the periphery of the active site, and the position 214, 287, 329, 354 are located more distant from the active site.

In order to reveal if there are any fundamental differences in the phenotypes of the $\mathrm{H} 1 \mathrm{~N} 1$ viruses, we then selected certain wild-type and H275Y mutant viruses from both the BR07-like and NC99-like lineages for subsequent characterization in vivo and in vitro. We chose viruses of NA genes of different molecular features for further comparison, with some being totally in agreement with the corresponding consensus residues at the ten positions and some possessing non-consensus residues at one or two positions. The alignments of the NA protein sequences of the selected H1N1 viruses with their corresponding consensus amino acid residues at ten positions are listed (Table 3-2). For example, the wild-type BR/59/07 and MEM/13/06 viruses, and the H275Y mutant NY/1692/09 and GA/20/06 viruses are fully in agreement with their corresponding consensus residues at all ten positions; while H275Y mutant HW/28/07, NY/3467/09, $\mathrm{MI} / 03 / 02$ viruses differ in their corresponding consensus residues at one or two positions respectively. 


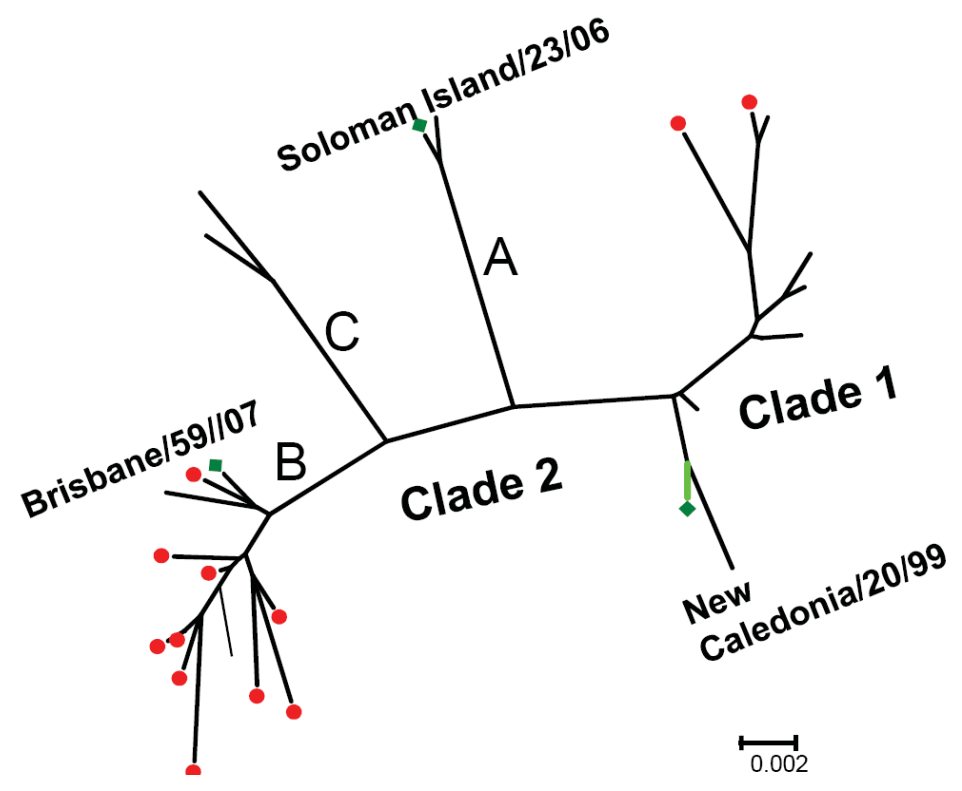

Figure 3-1. Evolution of NA genes of human seasonal H1N1 influenza viruses.

Representative strains of seasonal human H1N1 influenza viruses from 1999-2009 were chosen for construction of the tree. Seasonal H1N1 virus vaccine strains among the years are filled by green color, and viruses reported to carry the H275Y NA mutation are filled by red color. The phylogenetic analysis was based on alignment of coding sequences of the N1 NA gene. The tree was constructed by using the neighbor-joining method (Kimura two-parameter) with 1000 bootstrap replicates in the Mega4 program. Only bootstrap values $>50$ are shown. The bar represents 0.002 nucleotide substitutions per site. 
Table 3-1. Comparison of the amino acid consensus sequences of NA genes of human seasonal H1N1 viruses of different lineages.

\begin{tabular}{|c|c|c|c|c|c|c|c|c|c|c|c|c|}
\hline \multirow{2}{*}{$\begin{array}{l}\text { H1N1 virus } \\
\text { lineage }\end{array}$} & \multirow{2}{*}{$\begin{array}{l}\text { NA genotype } \\
(275 \text { position })^{a}\end{array}$} & \multicolumn{11}{|c|}{ NA consensus amino acid residue $\mathrm{e}^{\mathrm{b}}$} \\
\hline & & 275 & 45 & 78 & 214 & 222 & 234 & 249 & 287 & 329 & 344 & 354 \\
\hline \multirow[t]{2}{*}{$\mathrm{BR} / 59 / 07-$ like } & wild-type & $\mathrm{H}$ & $\mathrm{N}$ & $\mathrm{W}$ & $\mathrm{G}$ & $\mathrm{Q}$ & $\mathrm{M}$ & $\mathrm{K}$ & $\mathrm{I}$ & $\mathrm{E}$ & $\mathrm{N}$ & $\mathrm{D}$ \\
\hline & H275Y mutant & Y & - & - & - & - & - & - & - & - & - & $\mathrm{G}$ \\
\hline \multirow[t]{2}{*}{ NC/20/99-like } & wild-type & $\mathrm{H}$ & $\mathrm{H}$ & $\mathrm{K}$ & $\mathrm{E}$ & $\mathrm{R}$ & V & $\mathrm{G}$ & $\mathrm{T}$ & K & $\mathrm{D}$ & $\mathrm{G}$ \\
\hline & H275Y mutant & $\mathrm{Y}$ & - & - & - & - & - & - & - & - & - & - \\
\hline
\end{tabular}

${ }^{a}$ Indicate the amino acid residue at the 275 position of NA protein.

" In the table, the symbol "." indicates the amino acid residue in the mutant viruses is the same as the residue in the wild-type virus in the group. 

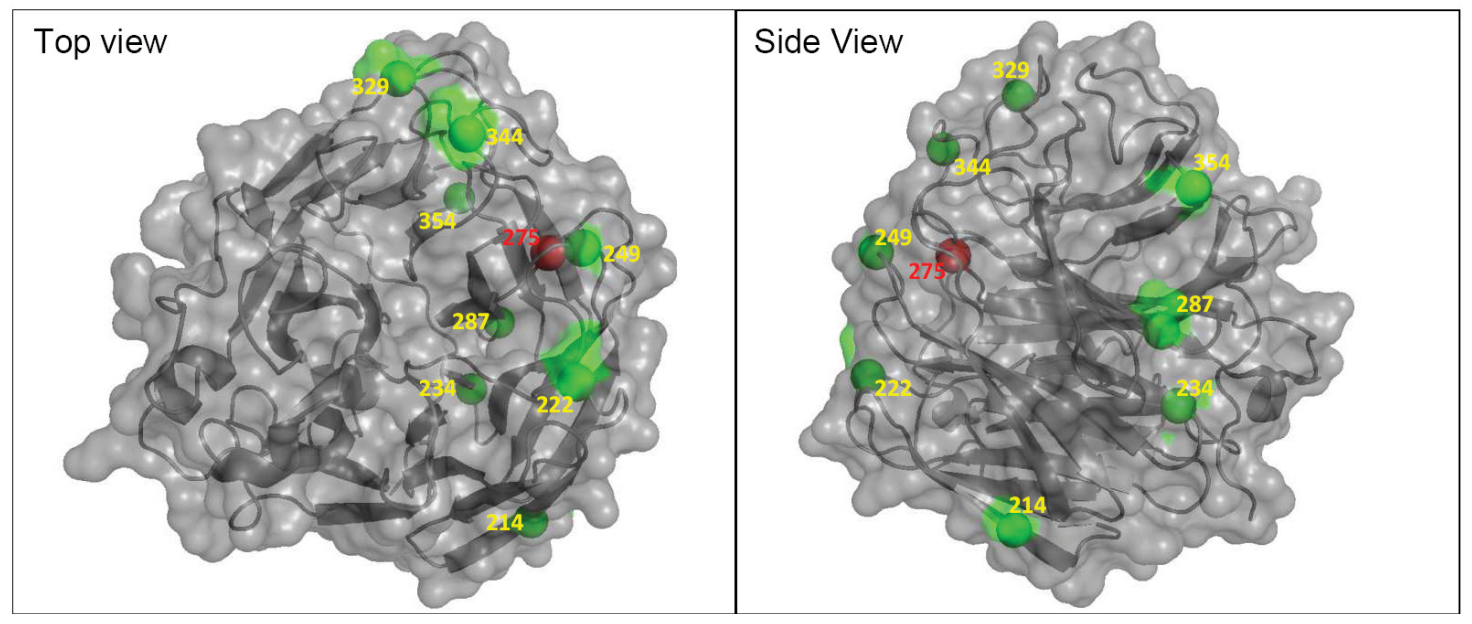

Figure 3-2. The structure of NA monomer (N1 subtype) showing the 275 position and the 10 positions at which the amino acid substitutions differentiate the NAs of NC99-like and BR07-like.

Surface-filling model of the neuraminidase protein of A/California/04/09 virus (PDB 3 NSS) shows the location of the 8 positions of amino acid residues listed in the Table 3 1. Position 45 and 78 are located in stalk region of NA molecular, which is no included in this protein structure. Both the top view and side view of the NA globular head were showed for the same 8 positions. The respective residues at the 10 positions are labeled in green and residue 275 is labeled in red which confers resistance to oseltamivir by the $\mathrm{H}$ to T mutation. The structure view was generated by PyMOL software. 
Table 3-2. Agreement of the NA genes of the H1N1 viruses with the respective NA censuses sequences of human H1N1 viruses of different lineages at the 10 amino acid positions plus the 275 position.

\begin{tabular}{|c|c|c|c|c|c|c|c|c|c|c|c|}
\hline \multirow{2}{*}{$\begin{array}{l}\text { H1N1 virus } \\
\text { lineages and strains } \\
\text { (genotype at NA } 275 \\
\text { position) }\end{array}$} & \multicolumn{11}{|c|}{ NA amino acid residue ${ }^{b}$} \\
\hline & 275 & 45 & 78 & 214 & 222 & 234 & 249 & 287 & 329 & 344 & 354 \\
\hline \multicolumn{12}{|l|}{ Seasonal BR07-like } \\
\hline wild-type consensus & $\mathbf{H}$ & $\mathbf{N}$ & $\mathbf{W}$ & $\mathbf{G}$ & $\mathbf{Q}$ & $\mathbf{M}$ & $\mathbf{K}$ & I & $\mathbf{E}$ & $\mathbf{N}$ & D \\
\hline $\mathrm{BR} / 59 / 07$ (wt) & - & - & - & - & - & - & - & - & - & - & - \\
\hline $\mathrm{CA} / 27 / 07(w t)$ & - & - & - & - & - & - & - & - & - & - & - \\
\hline H275Y variant consensus & $\mathbf{Y}$ & $\mathbf{N}$ & $\mathbf{W}$ & $\mathbf{G}$ & $\mathbf{Q}$ & $\mathbf{M}$ & $\mathbf{K}$ & I & $\mathbf{E}$ & $\mathbf{N}$ & $\mathbf{G}$ \\
\hline HW/28/06 (H275Y) & - & - & - & - & - & - & - & - & - & - & $\mathrm{D}$ \\
\hline NY/3467/09 (H275Y) & - & - & - & - & - & - & - & - & - & $\mathrm{D}$ & - \\
\hline NY/1692/09 (H275Y) & - & - & - & - & - & - & - & - & - & - & - \\
\hline NJ/15/07 (H275Y) & - & - & - & - & - & - & - & - & - & - & - \\
\hline \multicolumn{12}{|l|}{ Seasonal NC99-like } \\
\hline wild-type consensus & $\mathbf{H}$ & $\mathbf{H}$ & $\mathbf{K}$ & $\mathbf{E}$ & $\mathbf{R}$ & $\mathbf{V}$ & $\mathbf{G}$ & $\mathbf{T}$ & $\mathbf{K}$ & D & $\mathbf{G}$ \\
\hline MEM/13/06 (wt) & - & - & - & - & - & - & - & - & - & - & - \\
\hline GA/17/06 (wt) & - & - & - & - & - & - & - & - & - & - & - \\
\hline $\mathrm{MI} / 03 / 02(\mathrm{wt})$ & - & - & - & - & Q & - & - & - & - & $\mathrm{N}$ & - \\
\hline H275Y variant consensus & $\mathbf{Y}$ & $\mathbf{H}$ & $\mathbf{K}$ & $\mathbf{E}$ & $\mathbf{R}$ & $\mathbf{V}$ & $\mathbf{G}$ & $\mathbf{T}$ & $\mathbf{K}$ & D & $\mathbf{G}$ \\
\hline GA/20/06 (H275Y) & - & - & - & - & - & - & - & - & - & - & - \\
\hline MI/03/02 (H275Y) & - & - & - & - & Q & - & - & - & - & $\mathrm{N}$ & - \\
\hline TX/30/07 (H275Y) & - & - & - & - & - & - & - & - & - & - & - \\
\hline \multicolumn{12}{|l|}{2009 pandemic H1N1 } \\
\hline wild-type consensus & $\mathbf{H}$ & $\mathbf{Q}$ & $\mathbf{Q}$ & D & $\mathbf{N}$ & $\mathbf{V}$ & $\mathbf{G}$ & $\mathbf{E}$ & $\mathbf{N}$ & $\mathbf{N}$ & $\mathbf{G}$ \\
\hline DM/524/09 (wt) & - & - & - & - & - & - & - & - & - & - & - \\
\hline H275Y variant consensus & $\mathbf{Y}$ & $\mathbf{Q}$ & $\mathbf{Q}$ & $\mathbf{D}$ & $\mathbf{N}$ & $\mathbf{V}$ & $\mathbf{G}$ & $\mathbf{E}$ & $\mathbf{N}$ & $\mathbf{N}$ & $\mathbf{G}$ \\
\hline DM/528/09 (H275Y) & - & - & - & - & - & - & - & - & - & - & - \\
\hline
\end{tabular}


Table 3-2. (Continued).

${ }^{a}$ Indicates the amino acid residue at the 275 position of NA protein.

b In the table, the "-" symbol indicates the amino acid residue in the mutant viruses as same as the residue in the wild-type virus in the group. 


\section{Transmissibility of Different Seasonal H1N1 Viruses in Ferrets}

Transmissibility of wild-type and H275Y mutant BR07-like viruses in ferrets. Transmissibility is considered as one of the most important clinical relevant properties of influenza viruses, so we firstly assessed the transmissibility of the H1N1 viruses in a ferret model. Four strains of BR07-like viruses were studied. The BR/59/07 virus is a representative wild-type virus for this lineage, which is shown as the immediate ancestor for the majority of H275Y mutant viruses that emerged between 2007 2009 (Fig 3-1). The other three viruses share the same lineage as the BR/59/07 virus but harbor the NA H275Y resistance marker and carry different NA molecular features: the NA gene of $\mathrm{NY} / 1692 / 09$ virus is fully in agreement with the BR07-like consensus residues at the ten positions, but the NA genes of HW/28/07 and NY/3467/09 virus differ in their consensus residues at the 354 and 344 position respectively (Table 3-2).

We assessed the transmissibility of the four viruses in ferrets by both direct-contact route and respiratory-droplet route. In this model for each virus group, 3 ferrets were intra-nasally inoculated with the same infectivity amount of the virus (donor ferrets), then one naïve ferret was housed with each donor ferret in the same cage (direct-contact route) at day 1 post-inoculation (p.i.), and another naïve ferret was placed in an adjacent cage separated from the donor's cage by two layers of perforated divider (respiratory-droplet route) at the same time. Transmission of the viruses was assessed by detection of infection in recipient ferrets (nasal wash titers, clinical signs, and sero-conversion).

As regards to the donor ferrets, in each of the four virus groups, the intranasal inoculation of the viruses resulted in successful infection in all donor ferrets (3/3) by nasal wash titers and sero-conversion (Fig 3-3A, Table 3-3). The donor ferrets shed virus productively with comparable peak virus titers and shedding duration among all 4 virus groups. The donor virus shedding at dayl post infection (p.i) were further analyzed, because dayl p.i was the time when the recipient ferrets were housed with the donors, and the level of the donor shedding might indicate the level of environmental virus exposure to the recipient ferrets at the time. Among all 4 virus groups, at day 1 p.i only donor ferrets in the NY/3467/09 virus group had slightly lower shedding (1.5 $\log _{10} \mathrm{TCID}_{50}$ lower) than the wild-type virus group, and the other 2 virus groups had shedding titers comparable to the wild-type virus group (Fig 3-3A, Table 3-3).

Regarding to the direct-contact recipient ferrets, in each of the four virus groups, all the co-caged recipient ferrets (3/3) acquired infection from their donor ferrets through direct-contact transmission route, as shown by productive virus shedding over time and confirmed by sero-conversion in the each ferret (Fig 3-3A, Table 3-3). But the efficiency of acquisition of infection at day 1 post contact (p.c) was not entirely the same among the different groups (Fig 3-3B, Table 3-3). In the wild-type BR/59/07 virus group all 3/3 ferrets acquired infection at day 1 p.c with high virus titers. In the HW/28/07 virus group, only $2 / 3$ ferrets acquired infection at day 1 p.c with one ferret of lower titer than the wild-type group ( $\sim 2 \log _{10} \mathrm{TCID}_{50}$ lower). In the NY/3467/09 virus group, $3 / 3$ ferrets acquired infection at day 1 p.c with one ferret of lower titer than the wild-type group $(\sim 2$ 



Figure 3-3. Transmissibility of the seasonal H1N1 influenza viruses of two lineages in ferrets.

(A) The virus titer in the nasal wash samples from each donor (black), direct-contact (DC) recipient (blue), and respiratory-droplet (RD) recipient ferret (red) over the sample collection time of 2 weeks. Each graph represents each different virus. The donor ferret was housed with recipient ferrets at day 1 p.i. (B) The virus titers at day 1 p.i in nasal wash samples of the DC ferrets in different virus groups. Each dot represents individual donor ferret in the virus group. (C) The virus titers in the nasal wash samples of first detection of virus in the RD ferrets in different virus group. The day p.i of first detection of virus in each group is indicated. $* \mathrm{p}<0.05$ by $\mathrm{t}$ test when compared with MEM13 group. 
Table 3-3. Detection of virus replication and sero-conversion in ferrets during transmission of the H1N1 influenza viruses.

\begin{tabular}{|c|c|c|c|c|c|c|c|c|c|}
\hline \multirow{2}{*}{$\begin{array}{l}\text { H1N1 viruses } \\
\text { (NA genotype) }^{\mathrm{a}}\end{array}$} & \multicolumn{3}{|c|}{ Inoculated donors } & \multicolumn{3}{|c|}{ Direct-contact recipients } & \multicolumn{3}{|c|}{ Respiratory-droplet recipients } \\
\hline & $\begin{array}{c}\text { Virus } \\
\text { shedding }^{\mathrm{b}}\end{array}$ & $\begin{array}{c}\text { Duration } \\
\text { of } \\
\text { shedding }^{\mathrm{c}}\end{array}$ & $\underset{\text { titer }^{d}}{\text { Serum HI }}$ & $\begin{array}{c}\text { Virus } \\
\text { shedding }^{\mathrm{b}}\end{array}$ & $\begin{array}{c}\begin{array}{c}\text { Duration } \\
\text { of } \\
\text { shedding }\end{array} \\
\end{array}$ & $\underset{\text { titer }^{d}}{\text { Serum HI }}$ & $\begin{array}{c}\text { Virus } \\
\text { shedding }^{\mathrm{b}}\end{array}$ & $\begin{array}{c}\text { Duration } \\
\text { of } \\
\text { shedding }\end{array}$ & $\underset{\text { titer }^{\mathrm{d}}}{\text { Serum HI }}$ \\
\hline \multicolumn{10}{|l|}{ BR/59/07-like } \\
\hline $\mathrm{BR} / 59 / 07$ (wt) & $\begin{array}{c}3 / 3 \\
(6.3 / 7.1)\end{array}$ & $\begin{array}{c}6 \\
(6,6,6)\end{array}$ & $\begin{array}{l}2560,1280 \\
1280\end{array}$ & $\begin{array}{c}3 / 3 \\
(3.0 / 6.9)\end{array}$ & $\begin{array}{c}7 \\
(7,7,7)\end{array}$ & $\begin{array}{l}2560,2560 \\
1280\end{array}$ & $\begin{array}{l}2 / 3 \\
(6.0)\end{array}$ & $\begin{array}{c}5 \\
(5,5)\end{array}$ & $\begin{array}{l}320,1280 \\
\quad<10\end{array}$ \\
\hline HW/28/06 (H275Y) & $\begin{array}{c}3 / 3 \\
(6.3 / 7.6)\end{array}$ & $\begin{array}{c}6 \\
(6,6,6)\end{array}$ & $\begin{array}{l}2560,2560 \\
1280\end{array}$ & $\begin{array}{c}3 / 3 \\
(1.8 / 7.2)\end{array}$ & $\begin{array}{c}6.3 \\
(5,7,7)\end{array}$ & $\begin{array}{l}2560,2560 \\
\quad 2560\end{array}$ & $\begin{array}{c}2 / 3 \\
(6.25)\end{array}$ & $\begin{array}{c}5 \\
(5,5)\end{array}$ & $\begin{array}{c}1280 \\
2560,<10\end{array}$ \\
\hline NY/3467/09 (H275Y) & $\begin{array}{c}3 / 3 \\
(5.3 / 8.0)\end{array}$ & $\begin{array}{c}6 \\
(6,6,6)\end{array}$ & $\begin{array}{l}640,640 \\
1280\end{array}$ & $\begin{array}{c}3 / 3 \\
(2.9 / 7.1)\end{array}$ & $\begin{array}{c}7 \\
(7,7,7)\end{array}$ & $\begin{array}{l}1280,2560 \\
1280\end{array}$ & $\begin{array}{l}2 / 3 \\
(6.1)\end{array}$ & $\begin{array}{c}6 \\
(7,5)\end{array}$ & $\begin{array}{l}640,640 \\
\quad<10\end{array}$ \\
\hline NY/1692/09 (H275Y) & $\begin{array}{c}3 / 3 \\
(7.0 / 7.2)\end{array}$ & $\begin{array}{c}6 \\
(6,6,6)\end{array}$ & $\begin{array}{l}2560,1280 \\
2560\end{array}$ & $\begin{array}{c}3 / 3 \\
(4.6 / 6.3)\end{array}$ & $\begin{array}{c}5.7 \\
(5,5,7)\end{array}$ & $\begin{array}{l}1280,2560 \\
1280\end{array}$ & $\begin{array}{c}2 / 3 \\
(5.9)\end{array}$ & $\begin{array}{c}5 \\
(5,5)\end{array}$ & $\begin{array}{c}2560 \\
2560,<10\end{array}$ \\
\hline \multicolumn{10}{|l|}{ rgBR/59/07 viruses } \\
\hline $\mathrm{rgBR} / 59 / 07-\mathrm{NA}^{\mathrm{H} 275 \mathrm{Y}}$ & $\begin{array}{c}3 / 3 \\
(5.8 / 7.1)\end{array}$ & $\begin{array}{c}6 \\
(6,6,6)\end{array}$ & $\begin{array}{l}2560,2560 \\
2560\end{array}$ & $\begin{array}{c}3 / 3 \\
(1.2 / 7.1)\end{array}$ & $\begin{array}{c}5.7 \\
(5,5,7)\end{array}$ & $\begin{array}{l}2560,640 \\
2560\end{array}$ & $\begin{array}{c}2 / 3 \\
(6.75)\end{array}$ & $\begin{array}{c}6 \\
(5,7)\end{array}$ & $\begin{array}{c}2560 \\
1280,<10\end{array}$ \\
\hline $\mathrm{rgBR} / 59 / 07-\mathrm{NA}^{\mathrm{H} 275 \mathrm{Y}+\mathrm{D} 354 \mathrm{G}}$ & $\begin{array}{c}3 / 3 \\
(5.9 / 7.1)\end{array}$ & $\begin{array}{c}6 \\
(6,6,6)\end{array}$ & $\begin{array}{c}2560,1280 \\
2560\end{array}$ & $\begin{array}{c}3 / 3 \\
(2.9 / 7.2)\end{array}$ & $\begin{array}{c}7 \\
(7,7,7)\end{array}$ & $\begin{array}{c}2560,1280 \\
2560\end{array}$ & $\begin{array}{c}2 / 3 \\
(6.75)\end{array}$ & $\begin{array}{c}7 \\
(7,7)\end{array}$ & $\begin{array}{c}1280 \\
1280,<10\end{array}$ \\
\hline \multicolumn{10}{|l|}{ NC/20/99-like } \\
\hline MEM/13/06 (wt) & $\begin{array}{c}3 / 3 \\
(6.6 / 8.0)\end{array}$ & $\begin{array}{c}6 \\
(6,6,6)\end{array}$ & $\begin{array}{c}2560,2560 \\
2560\end{array}$ & $\begin{array}{c}3 / 3 \\
(3.7 / 7.4)\end{array}$ & $\begin{array}{c}7 \\
(7,7,7)\end{array}$ & $\begin{array}{c}2560,2560 \\
2560\end{array}$ & $\begin{array}{c}2 / 3 \\
(6.1)\end{array}$ & $\begin{array}{c}5 \\
(5,5)\end{array}$ & $\begin{array}{c}1280 \\
1280,<10\end{array}$ \\
\hline MI/03/02 (H275Y) & $\begin{array}{c}3 / 3 \\
\left(5.1^{*} / 6.9\right)\end{array}$ & $\begin{array}{c}6 \\
(6,6,6)\end{array}$ & $\begin{array}{c}640,1280 \\
1280\end{array}$ & $\begin{array}{c}3 / 3 \\
\left(0.3^{*} / 6.7\right)\end{array}$ & $\begin{array}{c}6.3 \\
(7,5,7)\end{array}$ & $\begin{array}{c}640,1280 \\
1280\end{array}$ & $\begin{array}{c}1 / 3 \\
(6.3)\end{array}$ & $\begin{array}{c}3 \\
(3)\end{array}$ & $\begin{array}{c}320,<10 \\
\quad<10\end{array}$ \\
\hline GA/20/06 (H275Y) & $\begin{array}{c}3 / 3 \\
\left(3.5^{*} / 6.8\right)\end{array}$ & $\begin{array}{c}6 \\
(6,6,6)\end{array}$ & $\begin{array}{c}640,1280 \\
1280\end{array}$ & $\begin{array}{c}3 / 3 \\
\left(0.7^{*} / 6.2\right)\end{array}$ & $\begin{array}{c}7 \\
(9,5,7)\end{array}$ & $\begin{array}{c}640,640 \\
1280\end{array}$ & $\begin{array}{c}1 / 3 \\
(7.0)\end{array}$ & $\begin{array}{c}5 \\
(5)\end{array}$ & $\begin{array}{c}320,<10 \\
<10\end{array}$ \\
\hline
\end{tabular}


Table 3-3. (Continued).

${ }^{a}$ Indicates the amino acid residue genotype at the 275 position of NA protein either as wt (wild-type H275) or H275Y mutation.

${ }^{\mathrm{b}}$ Number of virus-shedding animals/total number. Numbers in parentheses indicate mean virus titer at day 1 p.i / mean peak virus titer in nasal wash samples $\left(\log _{10} \mathrm{TCID}_{50} / \mathrm{ml}, \mathrm{n}=3\right)$.

${ }^{c}$ The number of the days from the first day of detection of virus titer till the last day of detection of virus titer. Numbers in parentheses indicate days for each individual ferret in the group.

${ }^{\mathrm{d}}$ Hemagglutination inhibition (HI) antibody titers to homologous virus in ferret serum on day 21 p.i. Numbers indicate the HI titer of each individual ferret in the group.

* Compared with the value in MEM/13/06 virus group, $\mathrm{p}<0.05$, $\mathrm{t}$ test. 
$\log _{10} \mathrm{TCID}_{50}$ ); while in the $\mathrm{NY} / 1692 / 09$ virus group, $3 / 3$ ferrets acquired infection and all had higher titers than the wild-type group $\left(\sim 1.5 \log _{10} \mathrm{TCID}_{50}\right.$ higher). Nevertheless, in spite of the differences in the acquisition of infection at day 1 p.c, all the direct-contact ferrets reached comparable levels of peak virus shedding among the different groups. The results suggested that both the BR07-like wild-type and H275Y mutant viruses were easily transmitted to recipient ferrets through direct-contact route, although the efficiency of the transmission of the $\mathrm{H} 275 \mathrm{Y}$ mutant viruses varied from slightly lower, higher, or similar efficiency than that of the wild-type BR/59/07 virus group.

As for the respiratory-droplet recipient ferrets, in each of the four virus groups, only $2 / 3$ recipient ferrets acquired infection from donor ferrets through respiratory-droplet transmission, as shown by productive virus shedding in the nasal washes and also confirmed by sero-conversion (Fig 3-3A, Table 3-3). 1/3 ferrets in each group did not acquire infection over the observation time, as indicated by lack of detection of virus shedding and sero-conversion. In general, compared with the direct-contact transmission, the respiratory-droplet transmission not only had relatively lower efficiency, but also had a greater lag time, varying from a delay of $2 \sim 6$ days as indicated by the first day of detection of virus shedding. Furthermore, the dynamics of respiratory-droplet transmission into $2 / 3$ ferrets was not entirely the same among the four virus groups (Fig 3-3C). The two ferrets acquired infection at day 3, and 7 p.c respectively in the wild-type BR/59/07 virus group as indicated by the first day of virus detection; day 3 and 5 p.c respectively in the HW/28/07 virus group and day 3 and 5 p.c respectively in the NY/3467/09 virus group, suggesting similar dynamics of transmission of these three viruses to that of the wild-type virus. In the NY/1692/09 virus group, both ferrets acquired infection at day 3 p.c, suggesting slightly faster transmission of this virus than that of the wild-type virus. Overall, the results together suggest that both the wild-type and $\mathrm{H} 275 \mathrm{Y}$ mutant BR07-like viruses were able to be transmitted to recipient ferrets through respiratory-droplet route, but with lower efficiency and a greater lag time than direct contact-route; the $\mathrm{H} 275 \mathrm{Y}$ mutant viruses were transmitted through respiratory-droplet route at similar efficiency or even slightly faster dynamics than the wild-type virus.

Regarding clinical signs, all the infected ferrets (by inoculation or transmission) were observed with slight body weight loss (less than $5 \%$ of original body weight), spontaneous sneezing, fluctuated body temperature, but no obvious fever during the time of infection (data not shown). Among ferrets of the four virus groups there was no significant difference observed in these clinical signs, suggesting that both wild-type and H275Y mutant of BR07-like viruses caused a similar course of the disease with similar severity of the disease.

In summary, these results suggested that compared to the wild-type BR07-like virus, the BR07-like H275Y mutant viruses were transmitted among ferrets by both direct-contact and respiratory-droplet routes at similar efficiency, with subtle variations in dynamics depending on the viruses; the $\mathrm{H} 275 \mathrm{Y}$ mutant viruses also caused a similar course of disease with similar severity in donor and recipient ferrets as the wild-type virus. 
Transmissibility of reverse-genetics BR/59/07 viruses carrying H275Y NA mutation or/and D354G mutation. Reverse-genetics influenza viruses contain homogeneous genetic backgrounds with specified mutations on segments, thus allow precise evaluation the impact of the mutations on the virus. We then assessed the impact of the $\mathrm{H} 275 \mathrm{Y}$ mutation on the transmissibility of BR/59/07 virus by using reverse-genetics $\mathrm{BR} / 59 / 07$ ( $\mathrm{rgBR} / 59 / 07)$ viruses carrying different mutations. The two viruses were generated on the entire genetic background of the BR/59/07 virus. The first virus rgBR/59/07-NA ${ }^{\mathrm{H} 275 \mathrm{Y}}$ differs only at the NA H275Y mutation from the wild-type $\mathrm{BR} / 59 / 07$ virus, which is similar to the NA genotype of $\mathrm{HW} / 28 / 07$ virus with regards to the NA consensus residues (Table 3-2). The second virus rgBR/59/07-NA ${ }^{\mathrm{H} 275 \mathrm{Y}+\mathrm{D} 354 \mathrm{G}}$ had one more mutation $\mathrm{D} 354 \mathrm{G}$ in addition to $\mathrm{H} 275 \mathrm{Y}$, which is similar to the NA genotype of NY/1692/09 virus with regards to the NA consensus residues (Table 3-2). The reverse-genetics viruses did not have differences in other genes, which was very likely in those H1N1 virus isolates.

In both $\mathrm{H} 275 \mathrm{Y}$ mutant virus groups, all the inoculated donor ferrets shed virus productively, with day $1 \mathrm{p}$.i virus titers, peak virus titer and duration of virus shedding comparable to those in the wild-type BR/59/07 virus group, suggesting no difference in the virus replication in ferret upper respiratory tract among these two viruses and the wild-type virus (Fig 3-3A, Table 3-3).

With regards to direct-contact transmission, all recipient ferrets (3/3) in each group acquired infection from their donor ferrets, as indicated by productive virus shedding and sero-conversion (Fig 3-3A, Table 3-3), but the efficiency of acquisition of infection at day 1 p.c was distinct between the two groups (Fig 3-3B). In the $\mathrm{rgBR} / 59 / 07-\mathrm{NA}^{\mathrm{H} 275 \mathrm{Y}}$ virus group, only $2 / 3$ ferrets acquired infection at the time, with one ferret of lower virus titer than the wild-type virus group $\left(\sim 2 \log _{10} \mathrm{TCID}_{50}\right.$ lower) while in the $\mathrm{rgBR} / 59 / 07-\mathrm{NA}^{\mathrm{H} 275 \mathrm{Y}+\mathrm{D} 354 \mathrm{G}}$ virus group, $3 / 3$ ferrets acquired infection with titers approximate to those in the wild-type virus group. In spite of the shedding difference at day 1 p.c, all the receipt ferrets reached comparable peak virus shedding. The results suggested that the $\mathrm{H} 275 \mathrm{Y}$ mutation alone in the $\mathrm{BR} / 59 / 07$ virus minimally altered the efficiency of direct-contact transmission and only caused a slight delay at early transmission/infection stages, but the $\mathrm{H} 275 \mathrm{Y}$ and $\mathrm{D} 354 \mathrm{G}$ mutation together might negate such a delay and restore the same transmission efficiency as that of the wild-type $\mathrm{BR} / 59 / 07$ virus.

As for respiratory-droplet transmission, only $2 / 3$ ferrets in each virus group acquired infection. The efficiency of the transmission was the same as the wild-type virus group (Fig 3-3A, Table 3-3), but the dynamics of the transmission were slightly different (Fig 3-3C). In the rgBR/59/07-NA ${ }^{\mathrm{H} 275 \mathrm{Y}}$ virus group 2 ferrets acquired infection at day 3 , 7 p.c respectively as indicated by first day of detection of virus shedding, which was the same as those in wild-type BR/59/07 virus group, while in the $\mathrm{rgBR} / 59 / 07-\mathrm{NA}^{\mathrm{H} 275 \mathrm{Y}+\mathrm{D} 354 \mathrm{G}}$ virus group 2 ferrets acquired infection at day 3, 5 p.c respectively, which was slightly faster than those in the wild-type or the H275Y mutation virus groups. The results show that the H275Y NA mutation alone minimally affected the efficiency and dynamics of 
respiratory-droplet transmission of the $\mathrm{BR} / 59 / 07$ virus, and the $\mathrm{D} 354 \mathrm{G}$ and $\mathrm{H} 275 \mathrm{Y}$ mutations together might slightly facilitate the transmission.

In summary, by using the reverse-genetic viruses, we showed that the $\mathrm{H} 275 \mathrm{Y}$ mutation alone hardly compromised the productive transmission of the BR/59/07 virus by both direct-contact and respiratory-droplet routes, although the dynamics of the transmission could be slightly altered. The D354G, a NA mutation of high frequency $(>95 \%)$ in $\mathrm{H} 275 \mathrm{Y}$ mutant viruses, together with $\mathrm{H} 275 \mathrm{Y}$ mutation might slightly facilitate the virus transmission by both routes. Thus, the D354G mutation was somewhat beneficial for overall transmission of $\mathrm{rgBR} / 59 / 07$ virus with the $\mathrm{H} 275 \mathrm{Y}$ mutation alone. As for clinical signs in the infected ferrets, there was no apparent difference observed among the three virus groups. Notably, the efficiency and dynamics of transmission of the $\mathrm{rgBR} / 59 / 07-\mathrm{NA}^{\mathrm{H} 275 \mathrm{Y}}$ and $\mathrm{rgBR} / 59 / 07-\mathrm{NA}^{\mathrm{H} 275 \mathrm{Y}+\mathrm{D} 354 \mathrm{G}}$ viruses were in line with those of HW/28/08 and NY/1692/09 viruses respectively, which are of similar NA genotype with regards to the consensus residues.

Transmissibility of wild-type and $\mathrm{H} 275 \mathrm{Y}$ mutant NC99-like viruses in ferrets. In comparison to the transmissibility of the BR07-like H1N1 viruses, we then studied the transmissibility of three strains of NC-99-like viruses in the ferret model. MEM/13/06 virus is a representative wild-type virus for this lineage (Fig 3-1), which shares the same lineage as the NC/20/99 virus. Two other viruses also fall into the NC/99 lineage (Fig 3-1), but harbor the H275Y NA mutation and carry different NA molecular features. The NA gene of GA/20/06 virus is fully in agreement with the consensus residues at the ten positions, while the NA gene of MI/03/02 virus differs at the 222 and 344 positions from the NC99-like consensus residues (Table 3-2).

We evaluated the transmissibility of these viruses among ferrets by both direct-contact and respiratory-droplet route. The experimental settings and protocol were the same as described above for the BR/59/07-like viruses.

As for the donor ferrets, in each of the three virus groups, the intranasal inoculation of viruses resulted in successful infection in all donor ferrets $(3 / 3)$, as indicated by productive virus shedding and sero-conversion (Fig 3-3A, Table 3-3). The donor ferrets had comparable peak virus titers and virus shedding duration among all three virus groups, but both of the $\mathrm{H} 275 \mathrm{Y}$ mutant GA/20/06 and MI/03/02 virus groups had significantly lower average virus titers than the wild-type virus group at day $1 \mathrm{p} . \mathrm{i}$ $\left(\sim 3\right.$ and $1.5 \log _{10} \mathrm{TCID}_{50}$ lower respectively, $\left.\mathrm{p}<0.05\right)$. The results suggest that the two $\mathrm{NC} / 99$-like H275Y mutant viruses had significantly delayed virus replication in ferret upper respiratory tract compared with the wild-type virus.

With regards to direct-contact transmission in each of the three virus groups, all recipient ferrets $(3 / 3)$ acquired infection from their donor ferrets as indicated by virus shedding and sero-conversion (Fig 3-3A, Table 3-3), but the efficiency of acquisition of infection at day 1 p.c. was distinct among groups (Fig 3-3B). In the wild-type $\mathrm{MEM} / 13 / 06$ virus group, $3 / 3$ ferrets acquired infection at day 1 p.c with high titers, but in both the $\mathrm{GA} / 20 / 06$ virus and $\mathrm{MI} / 03 / 02$ virus groups only $2 / 3$ and $1 / 3$ ferrets respectively 
acquired infection at the time, with significantly lower virus titers than those in the wild-type virus group $\left(\sim 3 \log _{10}\right.$ TCID $_{50}$ lower, $\left.\mathrm{p}<0.05\right)$. The results suggested that both NC/99-like H275Y mutant viruses had significantly delayed direct-contact transmission than the wild-type virus.

Regarding to respiratory-droplet transmission, in the wild-type MEM/13/06 virus group, 2/3 recipient ferrets acquired infection from donor ferrets at day 3,5 p.c respectively as indicated by the first day of virus detection in nasal washes (Fig 3-3A, Table 3-3). However, in both GA/20/06 virus and MEM/13/06 virus groups only $1 / 3$ recipient ferrets acquired infection and the other two recipient ferrets were not able to acquire infection during the whole observation time, as indicated by lack of detection of virus shedding and sero-conversion. Moreover, in the two H275Y mutant virus groups, the one infected recipient ferret acquired infection at day 7, 9 p.c. respectively (Fig 3-3C). This was considerably more delayed than the day 3 or 5 p.c in the wild-type virus group. The results suggested that the NC99-like H275Y mutant viruses were transmitted with considerably less efficiency than the wild-type virus through respiratory droplet route.

In summary, the results suggested that compared to the wild-type virus, the replication of NC99-like $\mathrm{H} 275 \mathrm{Y}$ mutant viruses by inoculation in the ferret upper respiratory tract was significantly delayed at early stage, and transmission of the NC99-like H275Y mutant viruses in ferrets was considerably less efficient and slower than the wild-type virus by both direct-contact and respiratory-droplet routes.

Notably, when the NC99-like and BR07-like groups are compared, the transmissibility of the NC99-like wild-type virus was indistinguishable to its counterpart BR07-like wild-type virus, while the transmissibility of the NC99-like H275Y mutant viruses was remarkably reduced in terms of transmission efficiency and dynamics by both routes when compared with their counterpart BR07-like H275Y mutant virus.

\section{Growth Capability of Different Seasonal H1N1 Viruses in Cell Culture}

Virus growth capability is another important overall virus characteristic of influenza viruses, so we evaluated single-cycle growth of the BR07-like and NC99-like H1N1 viruses in NHBE cells. NHBE cells grow and differentiate on air-liquid interfaces and support influenza virus growth without the addition of TPCK trypsin, making them the best representation of epithelial cell lining in the respiratory tract. All the four BR07-like viruses used in the transmission study were evaluated for their growth capabilities. Two pairs of NC99-like viruses, which had NA genes only differing at the 275 position within each pair, were evaluated for virus growth capabilities. In NHBE cells, for the BR07-like virus group (Fig 3-4), all three H275Y mutant viruses eventually grew to peak titers comparable to the wild-type virus at 12 hours p.i, although one virus $\mathrm{HW} / 28 / 07$ had delayed growth. In contrast, for the two pairs of NC99-like viruses (Fig $3-4)$, both the H275Y mutant viruses only grow to titers significantly lower than their counterpart wild-type viruses by 12 hours p.i $\left(\sim 2 \log _{10}\right.$ TCID $_{50}$ lower, $\left.\mathrm{p}<0.05\right)$. 

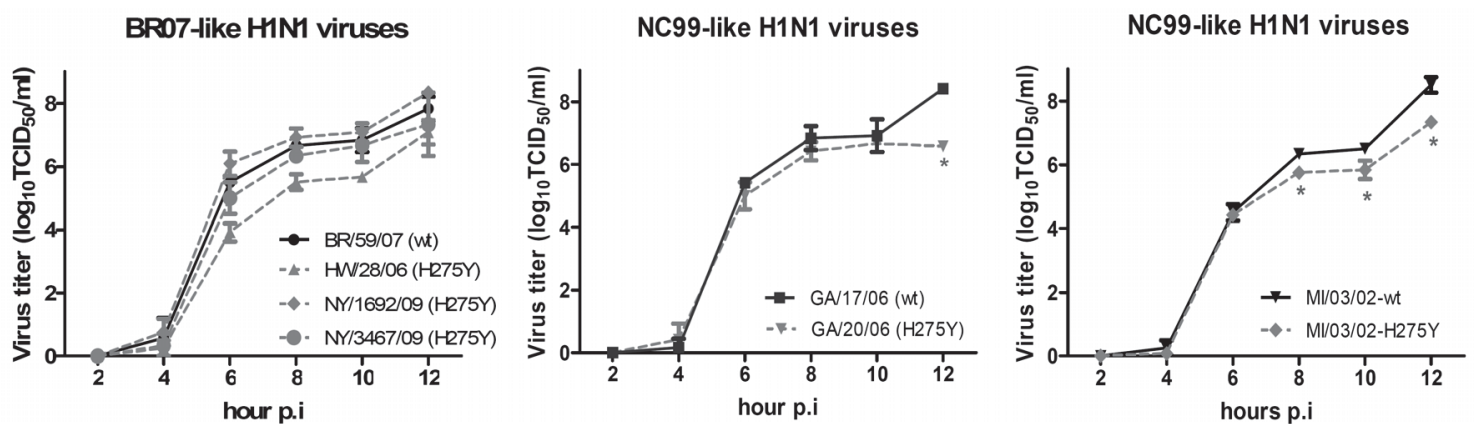

Figure 3-4. Growth capability of different H1N1 viruses in NHBE cells.

Single-cycle growth curves of different H1N1 viruses in MDCK cells were obtained by using an MOI of $\sim 2 \mathrm{PFU} /$ cell, respectively. Wild-type viruses are drawn in black solid lines and $\mathrm{H} 275 \mathrm{Y}$ mutant viruses are drew in grey dashed lines. Virus in the supernatant was titrated in MDCK cells and expressed as $\log _{10} \mathrm{TCID}_{50} / \mathrm{ml}$ at the indicated time post-infection (p.i). Each point represents the mean value \pm SD from three replicates. $* p<0.05$ by $t$ test, compared with the respective wild-type virus at the same time point. 
We also examined the plaque morphology by these H1N1 viruses in MDCK cells. Most viruses showed heterogeneous plaque sizes, and overall, the BR07-like viruses generated bigger plaques than the NC99-like viruses regardless of the H275Y mutation (data not shown), which is in agreement with previous reports on plaque morphology of H1N1 viruses (61).

Taken together, the results showed that in the cells, the growth capability of the BR07-like H275Y mutant viruses was approximate to their counterpart wild-type virus although with variations. In contrast, the growth capability of the NC99-like H275Y mutant viruses was considerably attenuated when compared with their counterpart wild-type viruses.

\section{NA Enzyme Activity, Protein Expression and Enzyme Kinetics of Different Seasonal H1N1 Viruses in vitro}

Generation and characterization of epitope-tagged recombinant NA proteins. In order to understand if the difference in transmissibility and infectivity of the characterized H1N1 viruses was associated to the NA enzyme function in the viruses, we assessed the NA enzyme activities of the BR07-like and NC99-like H1N1 viruses in vitro using a fluorescence-based substrate. When the whole virus particles standardized by the same infectivity were used for the assay, the results showed no significant difference of NA activity among different lineages, and failed to give any discernable trend among different groups (data not shown), which is in agreement with a previous report that no difference was found among the NA activities of wild-type and H275Y mutant H1N1 viruses (68). We speculated that the reason that such results were not informative could root in two major flaws about using whole virus particles for the assay: 1) virus particle quantitation can never be accurate enough and the errors masked the potential difference, as replication defective particles cannot be measured in infectivity assays and the infectivity assays by themselves are inaccurate; 2) the amount of virus surface NA protein was not counted into the analysis even if the virus particles were standardized, as different amount of NA proteins can be incorporated into the virus particle driven by different $\mathrm{M}$ proteins or different expression levels of the NA protein themselves. In order to overcome both potential flaws, we sought to measure enzyme activity of the NA proteins rather than the whole virus particles, which need antibodies to recognize the NA proteins. Due to the lack of one kind of anti-NA antibodies that can recognize all the NA variants with the same binding affinity, we generated the recombinant NA proteins with the same epitope tag, which allow quantitation of different NA protein variants for the following activity assay. We chose the HA epitope tag for the recombinant NA protein as it was reported previously (67).

We firstly assessed if the addition of the epitope tag to either NA protein terminus could affect the protein expression in cells (Fig 3-5). We generated the un-tagged, C-terminal tagged and N-terminal tagged recombinant proteins of the NA of BR59/07 virus and compared their expression levels (Fig 3-5A). First off, the expression levels of either tagged proteins were in proportion to the amounts of the plasmids transfected into 
A
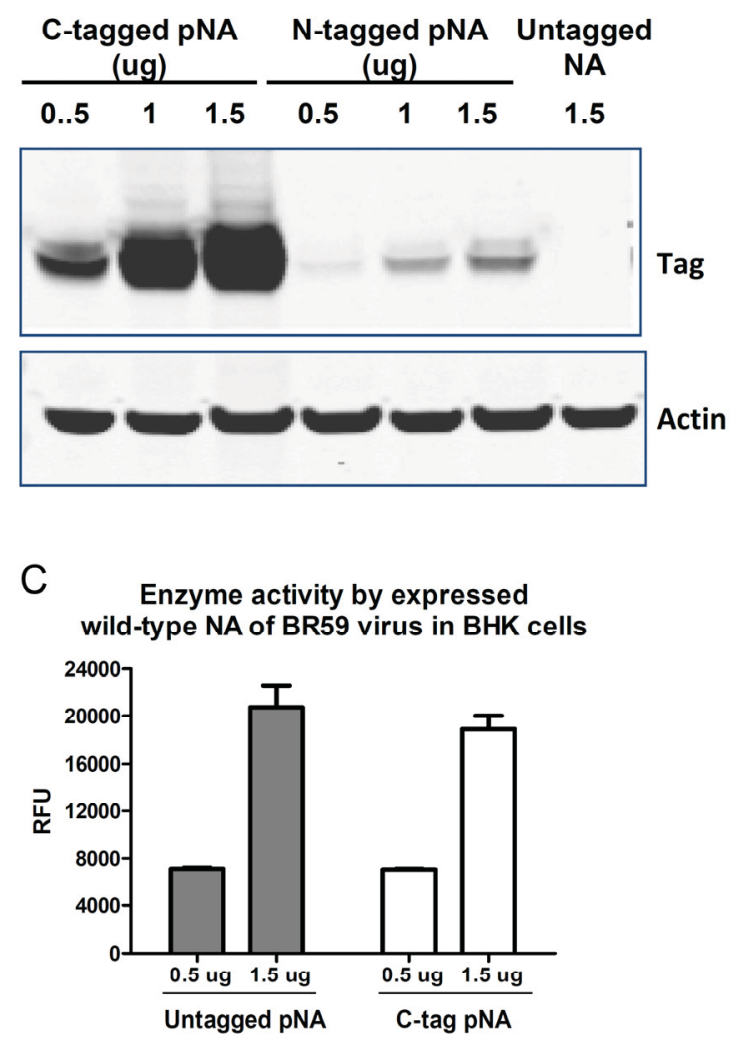

B

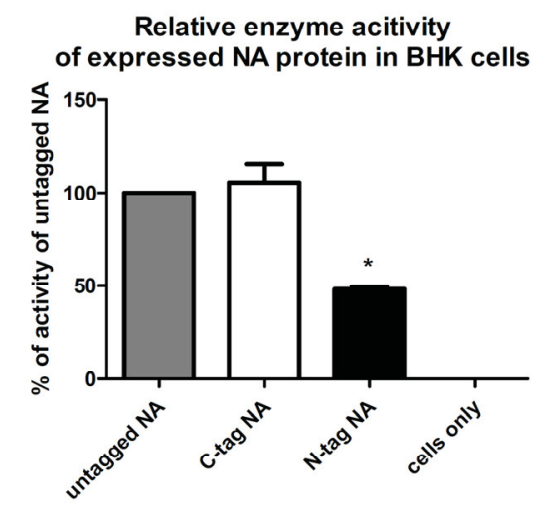

D

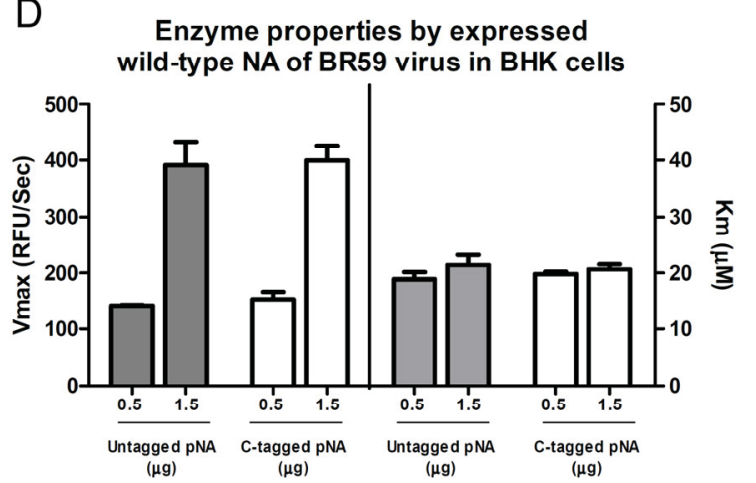

Figure 3-5. Generation and characterization of epitope-tagged recombinant NA protein of $B R / 59 / 07$ virus.

(A) The detection of different tagged recombinant BR/59/07-NA protein in BHK cells by western blot. The amounts of respective plasmids for transfection are labeled accordingly. The upper panel is the detection by anti-HA tag antibodies, and the lower is by anti-Actin antibodies. (B) The NA enzyme activity of the different tagged recombinant proteins in cells expressed by $0.5 \mu \mathrm{g}$ respective plasmids for transfection. The data were normalized to the untagged protein $(100 \%)$. ${ }^{*} \mathrm{p}<0.05$ by $\mathrm{t}$ test compared the untagged NA group. (C) The enzyme activity exhibited by untagged or C-terminal tagged proteins expressed by 0.5 and $1.5 \mu$ g respective plasmids. (D) The enzyme kinetics (Vmax at left $\mathrm{Y}$ axis and $\mathrm{Km}$ at right $\mathrm{Y}$ axis) exhibited by untagged or $\mathrm{C}$-terminal tagged proteins expressed by 0.5 and $1.5 \mu \mathrm{g}$ respective plasmids. 
the cells. Secondly, the expression level of the N-terminal tagged NA protein was constantly much lower than that of the $\mathrm{C}$-terminal tagged protein based on the density of the protein bands. The expression levels of the $\mathrm{C}$-terminal tagged and untagged NA protein cannot be directly compared by protein detection, but NA enzyme activity measurement showed that the two proteins exhibited approximately equivalent activities (Fig 3-5B), suggesting equivalent protein expression levels between the two proteins. In contrast, the N-terminal tagged protein retained a significantly lower amount of activity than the untagged and the C-terminal tagged proteins $(\mathrm{p}<0.05)$ (Fig 3-5B), which is in agreement with the protein detection results (Fig 3-5A). The comparison suggested that the $\mathrm{C}$-terminal HA epitope tag did not alter the level of the NA protein expression and activity, so the $\mathrm{C}$-terminal epitope tag was chosen for generation of all recombinant NA proteins.

Next, we examined the surface accumulation of the C-terminal tagged NA protein of BR/59/07 virus. During the life cycle of an influenza virus, the expressed NA proteins are reportedly transported to the cell surface to be incorporated into nascent virus particles. In order to make the results of NA protein expression mostly relevant to the levels of NA proteins incorporated into virus particles, we assessed the cell surface accumulation of the recombinant NA proteins. First of all, in the protein blot images, a relatively faint band was consistently found to accompany the major protein band (in either $\mathrm{C}$ - or N-terminal tagged protein images), and the density of the second band was always in proportion with its corresponding major band (Fig 3-5A). Considering their slightly higher molecular weight, presumably, the second protein bands could be the glycosylated NA proteins being transported to the cell surface. Secondly, we examined the cell surface accumulation of the NA proteins by flowcytometry (Fig 3-6). The results showed the accumulation of the C-terminal tagged NA proteins at the cell surface, and the accumulation intensity was in proportion to the amounts of the plasmids transfected into the cells.

Furthermore, we characterized the function of the C-terminal tagged NA protein of $\mathrm{BR} / 59 / 07$ virus in comparison to the untagged NA protein. The enzyme activities exhibited by both proteins were proportionate with their protein expression levels (Fig 3-5C), which were proportionate to the amounts of the plasmids transfected into the cells (Fig 3-5C). The enzyme properties Km and Vmax were determined for both the recombinant proteins (Fig 3-5D). Km is the dissociation constant for enzyme binding to the substrate, which is intrinsic to the enzyme and independent of the about of protein presented in the reaction; Vmax reflects catalytic capability of the enzyme and is dependent on both the enzyme turnover rate (Kcat) and the amount of enzyme present in the reaction. The results showed that both the $\mathrm{C}$-terminal tagged, untagged NA proteins (Fig 3-5D), and whole virus particles exhibited comparable Km values (data not shown), despite different amounts of the proteins or virus particles used for the assays. Both the recombinant NA proteins exhibited comparable Vmax values, which were proportionate to their expression amounts (Fig 3-5A). The results suggested that the C-terminal tagged NA protein retained the enzyme properties indistinguishable to the untagged NA protein, and could represent the original NA protein properly. 




Figure 3-6. The cell surface protein accumulation of C-terminal tagged BR07-NA protein.

The detection of the surface accumulation of the BR07-NA protein by flowcytometry. The different lines indicate the protein accumulation expressed by different amount of plasmids for transfection, which was, from left to right, $0,0.1,0.5,1.5 \mu \mathrm{g}$ of the plasmids into cells. 
Enzyme activities of recombinant NA proteins of H1N1 viruses in vitro. Next, we generated and compared the $\mathrm{C}$-terminal tagged recombinant NA proteins of different H1N1 viruses. All of the data were normalized to the activity of wild-type NA of the $\mathrm{BR} / 59 / 07$ virus (BR/59/07-NA) (Fig 3-7A). In the BR07-like group, the 5 different $\mathrm{H} 275 \mathrm{Y}$ mutant NA proteins exhibited enzyme activities approximate to the BR/59-NA protein with small variations (Fig 3-7A); especially, the BR/59/07-NA ${ }^{\mathrm{H} 275 \mathrm{Y}}$ protein exhibited a marginal loss ( $20 \%$, data not shown separately) of enzyme activity compared with the BR/59/07-NA protein, which only differs from each other at the H275Y mutation. However, in the NC99-like group, not only the 3 different wild-type NA proteins exhibited much lower activity than their wild-type counterpart BR/59/07-NA protein in the BR07-like group, but also the 3 different $\mathrm{H} 275 \mathrm{Y}$ mutant NA proteins exhibited drastic losses of the activity $(\sim 50 \%)$ compared with the corresponding 3 wild-type NA proteins (Fig 3-7A). Noted, the highest enzyme activities in either the wild-type or H275Y mutant NA proteins in the NC99-like group were from the MI/03/02 viruses, which differ from the NC99-like consensus residues at the 222 and 344 positions (Table 3-2).

Taken together, the results showed that the enzyme activities of BR07-like H275Y mutant NAs were minimally impaired compared to that of BR07-like wild-type NA, but the enzyme activities of the NC99-like H275Y NAs were severely diminished compared to those of their corresponding wild-type NAs, which were inherently-low by themselves. The results suggested that the enzyme activities of the wild-type NAs of the two different lineages are inherently distinct in the first place, and the H275Y mutation had differential effects on enzyme activity of the NAs of the two different lineages.

Expression levels of recombinant NA proteins of H1N1 viruses in cells. Reduced enzyme activity of the NA proteins can directly result from a reduced expression level of the proteins in cells. We next examined the expression levels of the recombinant NA proteins of the H1N1 viruses (Fig 3-7B). The total expression levels of the NA proteins in cells were examined by western blot. In the BR07-like group, the respective expression of 5 different $\mathrm{H} 275 \mathrm{Y}$ NA proteins were similar to that of the wild-type BR/59/07-NA protein although with slight variations based on the density of the protein bands (Fig 3-7B). Especially, the BR/59/07-NA ${ }^{\mathrm{H} 275 \mathrm{Y}}$ protein showed minimal change of expression level compared with the BR/59/07-NA protein. However, in the NC99-like group each of the 3 H275Y mutant NA proteins showed greatly reduced expression levels compared with their counterpart wild-type NA proteins (Fig 3-7B). Additionally, when the NC99-like and BR07-like groups are compared, each of the 3 NC99-like wild-type NA proteins showed naturally lower protein expression levels than the wild-type BR/59/07-NA respectively, and the 3 NC99-like H275Y mutant NA proteins showed greatly diminished protein accumulation compared with the BR07-like NA proteins.

We further examined the cell surface accumulation of 4 pairs of NA proteins of different viruses (Fig 3-7C). In each pair, the two NA proteins only differ from each other at the 275 position. In the BR07-NA group, the protein accumulation profiles of the H275Y mutant NA proteins were overlapped together with that of the wild-type NA 


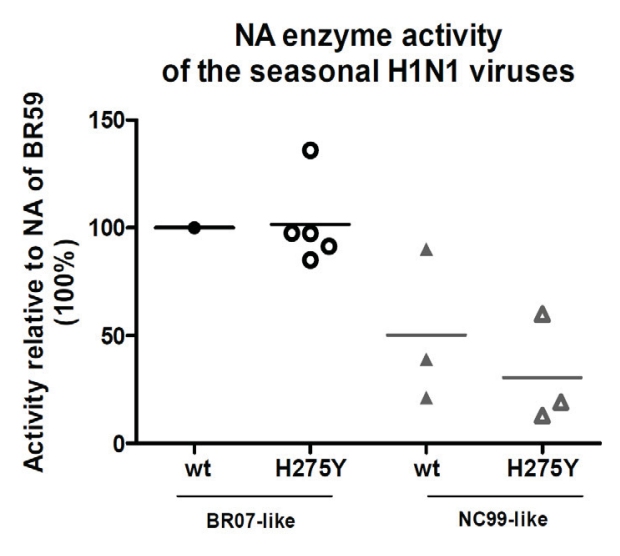

C
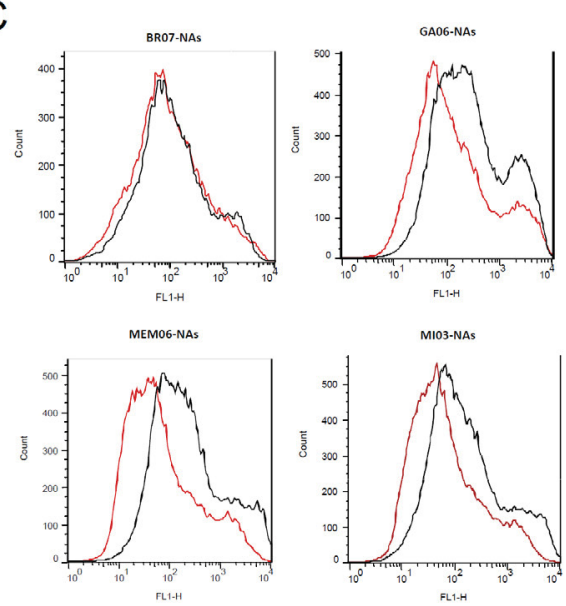

Figure 3-7. The NA enzyme activities, protein expression levels and enzyme Km values of different seasonal H1N1 influenza viruses.

(A) The enzyme activities of C-terminal tagged recombinant NA proteins of different H1N1 viruses. The data were normalized to the BR/59/07-NA. Each dot represents the mean value of three determinations of each different NA protein. The NA protein designations are entirely the same as in B panel. (B) The detection of the recombinant NA proteins of each virus by western blot. The upper panel is the detection by anti-HA tag antibodies, and the lower is by anti-Actin antibodies. (C) The detection of the cell surface accumulation of the 4 pairs of recombinant NA proteins by flowcytometry. Each panel is for each pair of NA proteins which only differs at 275 position in the pair (upper right $\mathrm{BR} / 59 / 07$ virus, upper left GA/17/06 and GA/20/06 viruses, lower right

$\mathrm{MEM} / 13 / 06$ virus, lower left MI/03/02 viruses) .In each panel, the black line and the red line indicate the wild-type and the $\mathrm{H} 275 \mathrm{Y}$ mutant NA protein respectively and two lines should be compared within each panel. (D) The NA enzyme Km value of different H1N1 viruses. Each dot represents the mean value of three determinations of each virus, although the data were not shown separately. 
protein (Fig 3-7C), suggesting that the surface accumulations of the two proteins were indistinguishable. However, in other three NC99-like NA groups, the profile of each $\mathrm{H} 275 \mathrm{Y}$ mutant NA protein greatly shifted to the left compared to its counterpart wild-type NA protein in the pair (Fig 3-7C), suggesting their greatly diminished cell surface accumulation. The results of cell surface NA accumulation and were consistent with the results of total NA protein expression in cells, suggesting that the lower cell surface NA accumulation could directly result from its lower protein expression in the cells.

Taken together, the results showed that both the total protein expression and the cell surface protein accumulation of the BR07-like H275Y mutant NAs were hardly altered compared with those of the BR07-like wild-type NA. However, both the total and surface protein accumulation of the NC99-like H275Y mutant NAs were greatly diminished compared with those of their counterpart NC99-like wild-type NAs. The results suggested that the protein expression level of the wild-type NAs of the two different lineages are naturally distinct in the first place, and the H275Y mutation had differential effects on the level of protein expression of the NAs of the two different lineages.

NA enzyme kinetics of the H1N1 viruses in vitro. Reduced enzyme activity of NA proteins can also result from reduced functional capability of the NA enzymes. Two enzyme kinetic parameters measure enzymatic functional capability: Km and Vmax. Km is an estimate of the dissociation equilibrium of enzyme binding to the substrate, and the reciprocal of $\mathrm{Km}$ approximates the enzyme affinity to substrate binding; Vmax reflects the catalytic activity of the enzyme and is determined by the reactive amino acid residues at enzyme active site and the amount of enzyme. Since none of the variant NA residues between the H1N1 viruses are located at the active site and the NA protein expression levels have been evaluated, only $\mathrm{Km}$ (the dissociation constant) was further determined for evaluation of the NA enzyme affinity to substrate binding.

In each of the BR07-like and NC99-like groups, all the H275Y mutant viruses exhibited higher average NA Km values than the wild-type viruses: $\sim 50 \mu \mathrm{M}$ vs $\sim 20 \mu \mathrm{M}$ in the BR07-like group and $\sim 200 \mu \mathrm{M}$ vs $100 \mu \mathrm{M}$ in the NC99-like group (Fig 3-7D), indicating lower substrate binding affinity in these $\mathrm{H} 275 \mathrm{Y}$ mutant NAs than those of their counterpart wild-type NAs. However, when the BR07-like and NC99-like groups were compared, the results showed the NA Km values of two groups were but at different levels, which were $\sim 100 \mu \mathrm{M}$ vs $20 \mu \mathrm{M}$ between the NC99-like and BR07-like wild-type viruses, and were $\sim 200 \mu \mathrm{M}$ vs $50 \mu \mathrm{M}$ between the $\mathrm{H} 275 \mathrm{Y}$ mutant viruses in two groups (Fig 3-7D), suggesting inherently-low enzyme affinity in the NC99-like NAs. Noted, the highest NA Km value in $\mathrm{H} 275 \mathrm{Y}$ mutant viruses in the BR07-like group was from NY/3467/09 virus, which differs from its consensus residues at the 344 position (Table $3-2$ ). The lowest NA Km values in either wild-type or H275Y mutant viruses in the NC99-like group were from the MI/03/02 viruses, which differ from their consensus residues at two positions (Table 3-2). 
Taken together, the results showed that the BR07-like H275Y mutant viruses had only slightly reduced NA enzyme affinities compared to their counterpart wild-type viruses due to the inherently-high level of NA affinity in this lineage. However, the NC99-like H275Y mutant viruses have greatly diminished NA affinities compared to their counterpart wild-type viruses due to the inherently-low level of NA affinity in this lineage. The results were consistent with but also further expanded previous reports $(63 ; 69 ; 70)$, suggesting that the enzyme affinity of the wild-type NAs of the two different lineages were inherently distinct in the first place, and the H275Y mutation had differential effects on the level of enzyme affinity of the NAs of two different lineages.

To summarize the in vitro characterization of the NAs of different H1N1 viruses, between the BR07-like and NC99-like groups, with regards to the wild-type NAs, BR07-like NAs had naturally higher enzyme activities than the NC99-like NAs, due to the higher level of NA protein expression and NA enzyme affinity. Within the BR07-like group, compared to the wild-type NA, the H275Y mutant NAs had minimally impaired enzyme activities because of the minimally reduced naturally-high protein expression and enzyme affinities. Within the NC99-like group, compared with the wild-type NAs, the $\mathrm{H} 275 \mathrm{Y}$ mutant NAs had greatly diminished enzyme activities, as a result of further reduced naturally-low NA protein expression and enzyme affinities. The results suggested that the $\mathrm{H} 275 \mathrm{Y}$ mutation commonly had a negative effect on the NA protein expression and enzyme affinity, but the degree of effects could be minimal as in BR07-like NAs, or be drastic as in NC99-like NAs. In other words, the BR07-like NAs had tolerance to the $\mathrm{H} 275 \mathrm{Y}$ mutation, but the NC99-like NAs had no tolerance to it.

In summary of the in vivo and in vitro phenotype characterization of different H1N1 viruses, in the BR07-like virus lineage, the wild-type NAs were naturally-high in quantity (protein expression) and quality (enzyme affinity), so they could tolerate the negative effects of the $\mathrm{H} 275 \mathrm{Y}$ mutation on the protein expression and enzyme affinity and the H275Y mutant NAs only had a marginal loss of NA activity; as a result, the $\mathrm{H} 275 \mathrm{Y}$ mutant viruses were minimally compromised in terms of virus infectivity and transmissibility, and were allowed in the BR07-like lineage. However, in the NC99-like virus lineage, the wild-type NAs were inherently-low in quantity and quality, so they were not able to tolerate the deleterious effects of the H275Y mutation on the protein expression and enzyme affinity and $\mathrm{H} 275 \mathrm{Y}$ mutant NAs had a great loss of NA activity; as a results, the $\mathrm{H} 275 \mathrm{Y}$ mutant viruses were severely compromised in terms of virus infectivity and transmissibility, thus they were extinct in the NC99-like lineage.

\section{Effects of the Single Amino Acid Substitutions in BR07-NA Gene (from BR07-like to NC99-like) on Protein Function and Expression}

Effects of NA variants of different enzyme activities on plaque morphology of the reverse-genetics viruses. Next, we sought to find the NA molecular basis for the distinct quantity and quality of NA proteins of the BR07-like and NC99-like lineages. We firstly chose two pairs of NA genes/proteins with one wild-type and another H275Y mutant to represent each lineage for subsequent in vitro characterization. The NA gene of 
wild-type BR/59/07 virus (BR07-NA) and its $\mathrm{H} 275 \mathrm{Y}$ variant acquired by mutagenesis at the 275 position (BR07-NA ${ }^{\mathrm{H} 275 \mathrm{Y}}$ ) were chosen to represent the BR07-like lineage. The NA genes of viruses GA/17/06 and GA/20/06 viruses (GA06-NA, GA06-NA ${ }^{\mathrm{H} 275 \mathrm{Y}}$ ) which naturally only differ at $\mathrm{H} 275 \mathrm{Y}$ mutation were chosen to represent the NC99-like lineage.

Since the two pairs of NAs (BR07 and GA06 pair) showed very contrasting levels of NA activity in vitro (Fig 3-5A, 2-6A), we further tested if the different levels of NA activity could affect the virus growth in terms of plaque size in MDCK cells. We generated the reverse genetic viruses by using the 7 segments (except the NA segment) from the BR/59/07 virus with the two pairs of NA genes respectively. Among the 4 reverse-genetics viruses, BR07-NA generated big clear plaques $(1.8 \mathrm{~mm})$, without apparent reduction of the plaque size by the presence of $\mathrm{H} 275 \mathrm{Y}$ mutation $\left(\mathrm{BR} 07-\mathrm{NA}^{\mathrm{H} 275 \mathrm{Y}}\right)(1.8 \mathrm{~mm})($ Fig 3-8A, B); in contrast, GA06-NA only generated pinpoint-like small plaques $(0.5 \mathrm{~mm})$, and even smaller plaques with the presence of $\mathrm{H} 275 \mathrm{Y}$ mutation $\left(\mathrm{GA} 06-\mathrm{NA}{ }^{\mathrm{H} 275 \mathrm{Y}}\right)(0.3 \mathrm{~mm})$ (Fig 3-8A, B). In these reverse-genetics viruses, the virus absorption and replication by the same viral 7 proteins were totally the same and only the release and spread of progeny virus by different NA proteins was different, thus the plaque sizes can be associated with different function levels of the NA variants during virus growth in the cells. The results suggested that the plaque sizes of the reverse-genetics viruses, which only differed at the NA genes, were positively correlated with the levels of their NA enzyme activities. In other words, the lower NA activity, the smaller plaque size, and vice versa.

Effects of the single amino acid substitutions (from BR07-like to NC99-like) in BR07-NA gene on plaque morphology of the reverse-genetics viruses. Based on the results that the level of NA activity can determine the plaque size of the reverse-genetics viruses, we took a "loss-of-activity" and "reduction-of-size" strategy to find the amino acid residues in the BR07-NA protein which are responsible for its high enzyme activity. We used the reduction of plaque size of reverse genetic viruses of NA variants as the first criteria to probe potential activity-reducing amino acid in the BR59-NA. There are only 10 amino acid substitutions differentiating between BR07-like and NC99-like NAs (Table 3-1). We substituted each of the 10 amino acid residues in the BR07-NA gene with the corresponding NC99-like consensus residues respectively, as N45H+W78K, G214R, Q222R, M234V, K249G, I287T, E329K, N344D, D354G (the 45 and 78 positions were placed together because they are the only two residues located in the stalk region of NA), with the absence or the presence of the $\mathrm{H} 275 \mathrm{Y}$ mutation respectively, then we generated the reverse genetics viruses by using the 18 variants of the BR07-NA gene. The plaque assay in MDCK cells showed that regardless of the presence of the H275Y mutation, the majority of the substitutions did not cause an appreciable change in the plaque size of the viruses (Fig 3-8C), and only 4 respective substitutions (Q222R, $\mathrm{M} 234 \mathrm{~V}, \mathrm{E} 329 \mathrm{~K}$ and N344D) resulted in significantly reduced plaque size in a different manner $(\mathrm{p}<0.05)$ (Fig 3-8C, Fig 3-9). Specifically, the Q222R and E329K caused drastically reduced plaque sizes of the viruses regardless of the $\mathrm{H} 275 \mathrm{Y}$ mutation $(0.7,0.8$ $\mathrm{mm}$ respectively and $0.6,0.7 \mathrm{~mm}$ respectively with the presence of the $\mathrm{H} 275 \mathrm{Y})$. The $\mathrm{M} 234 \mathrm{~V}$ or N344D alone caused a marginal change in the plaque sizes $(1.5,1.7 \mathrm{~mm}$ 
A

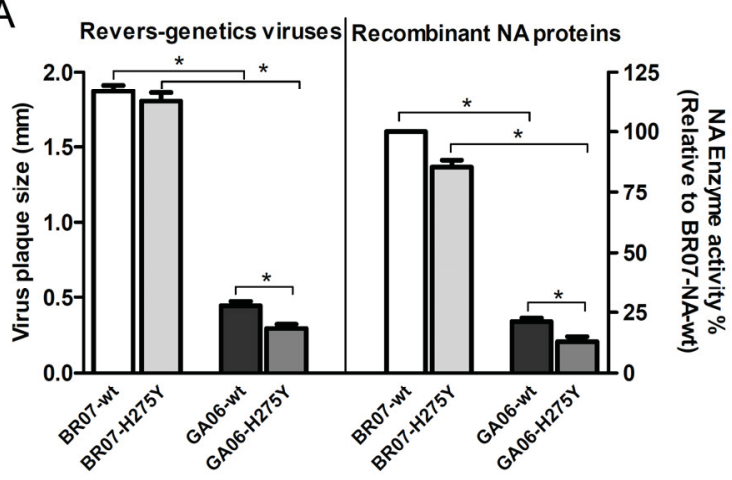

B



C Virus plaque sizes by different single mutations in the BR07-NA genes

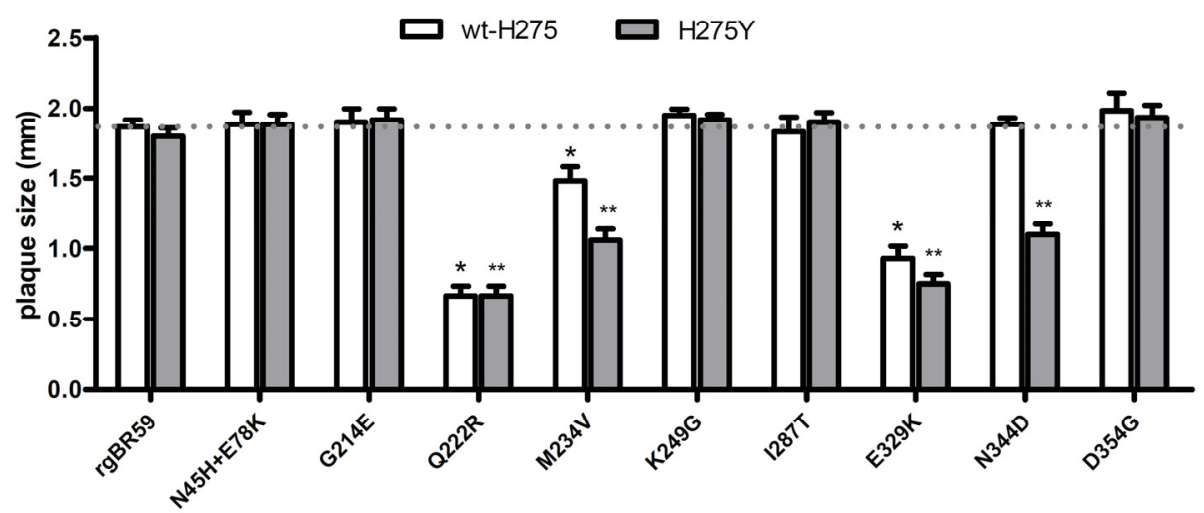

Figure 3-8. The plaque sizes of $\mathrm{rgBR} / 59 / 07$ viruses containing different NA gene variants.

(A) The plaque sizes (left $\mathrm{Y}$ axis) of rgBR/59/07 viruses using two pairs of NA gene variants (BR07 and GA06 pair), and the enzyme activities (right Y axis) of each pair of proteins. The diameters of 10 randomly selected plaques were measured. Values are mean plaque diameters $(\mathrm{mm}) \pm \mathrm{SD} * \mathrm{p}<0.05$ by $\mathrm{t}$ test between indicated groups. (B) The plaque morphology of the $\mathrm{rgBR} / 59 / 07$ ( $\mathrm{rgBR} / 59)$ viruses using two pairs of NA variants. (C) The plaque sizes of $\mathrm{rgBR} / 59 / 07$ viruses using 20 different NA variants of different single amino acid residue substitution with the absence (wt-H275) or the presence $(\mathrm{H} 275 \mathrm{Y})$ of the $\mathrm{H} 275 \mathrm{Y}$ mutation. The mutations are indicated in each group. $*, * * \mathrm{p}<0.05$ by $\mathrm{t}$ test when compared with rgBR59-wt-H275 or rgBR59-wt-H275Y respectively. 


\section{Wild-type viruses}

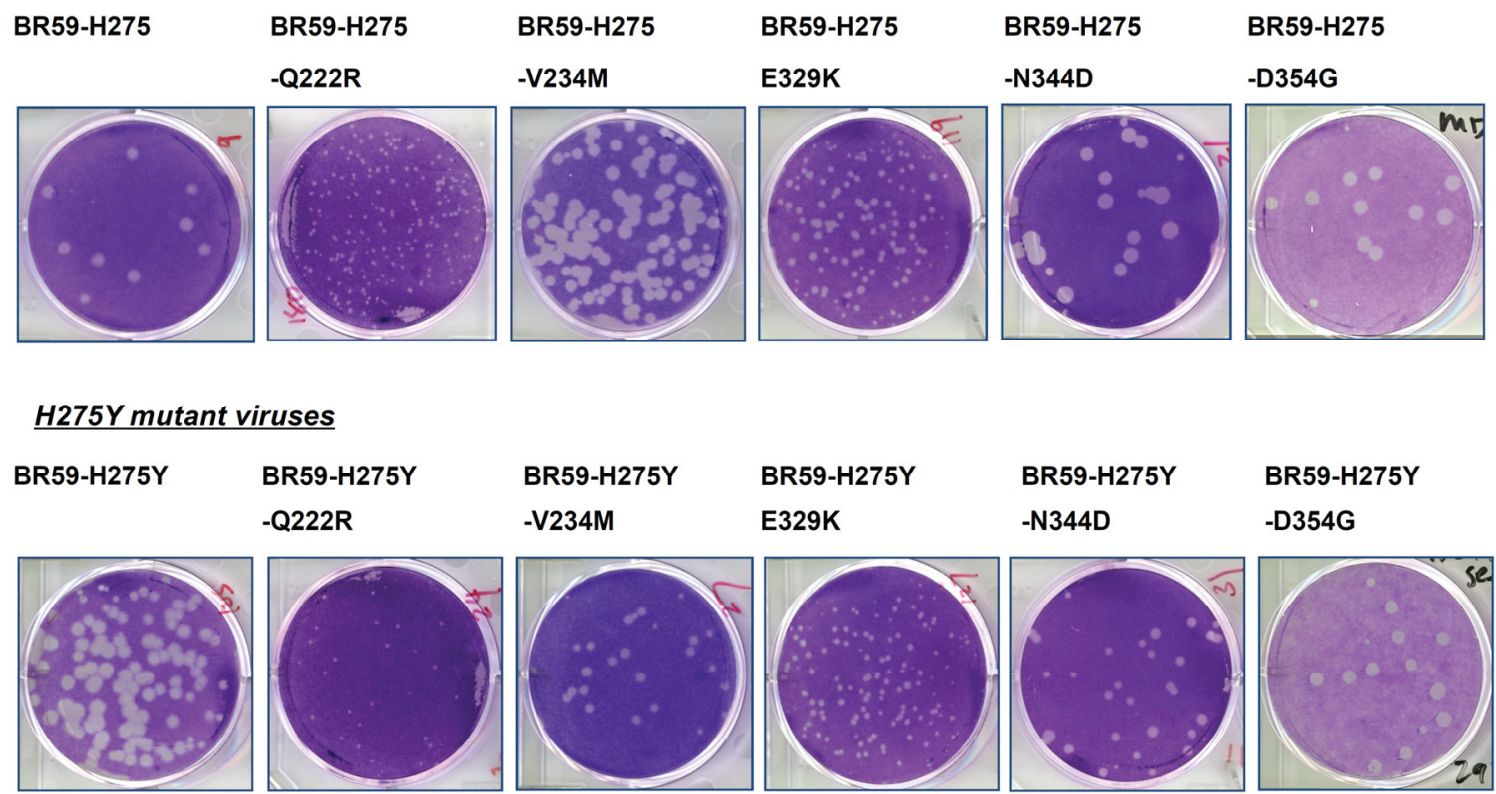

Figure 3-9. The plaque morphologies of $\mathrm{rgBR} / 59 / 07$ viruses of single amino acid residue substitutions in the $\mathrm{NA}$ gene.

The plaque morphology of rgBR/59/07 viruses using 10 different BR07-NA gene variants of different single amino acid residue substitution with the absence (wt-H275) or the presence (mut-H275Y) of the H275Y mutation. The mutation sites are indicated above in each image. 
respectively), but caused a greater reduction of the plaque sizes at the presence of the $\mathrm{H} 275 \mathrm{Y}$ mutation (1.0, $0.9 \mathrm{~mm}$ respectively). The results suggested that the 4 amino acid substitutions which caused notable change of the virus plaque sizes could potentially cause decrease of the NA activity in the viruses. We chose these 4 NA amino acid positions for further evaluations, but acknowledged that the other 6 substitutions might also exert certain effects on the NA activity, but not big enough to cause a detectable change in the virus plaque size. The NA position 354 exhibited unique amino acid residue reversion (Table 3-1), so this position also was included along with other 4 positions for future analysis.

Effects of the single amino acid substitutions (from BR07-like to NC99-like) in BR07-NA gene on protein function and expression. We identified 4 amino acid substitutions in the BR07-NA gene causing reduction of the virus plaque sizes, so we proceeded to evaluate if the four substitutions caused loss of activity in the NA proteins and by which mechanism. We determined the enzyme activities of the 12 variant BR07-NA proteins of the single amino acid substitutions (Fig 3-10A). First, the H275Y mutation alone in the BR07-NA protein only caused a small decrease of the enzyme activity; secondly, each of the substitutions (Q222R, M234V, E329K, N344D) in the BR07-NA caused a significant decrease of the enzyme activity with a greater magnitude at the presence of the $\mathrm{H} 275 \mathrm{Y}$ mutation $(\mathrm{p}<0.5)$, which were correlated with the reduced virus plaque sizes caused by these substitutions (Fig 3-8C). The D354G in the BR07-NA increased the enzyme activity which was opposite to other 4 substitutions (Fig 3-10C), although it did not cause a notable change in virus plaque size (Fig 3-8C).

We tested the effects of each of the 5 amino acid residue substitutions on the protein expression and enzyme affinity of BR07-NA (Fig 3-10B). First off, The H275Y mutation alone in the BR07-NA caused minimal change in the protein expression; two substitutions (Q222R, M234V) in the BR07-NA severely ablated the level of protein expression, even to a lower level with the presence of the H275Y mutation; the other 3 substitutions did not appear to affect the level of protein expression. Secondly, as regard to the effects on the NA enzyme affinity (Fig 3-10C);, the H275Y mutation alone in BR07-NA slightly increased the Km value three substitutions (Q222R, E329K, N344D) in the BR07-NA significantly raised the enzyme $\mathrm{Km}$ values $(\mathrm{P}<0.05)$, to a bigger degree with the presence of the $\mathrm{H} 275 \mathrm{Y}$ mutation, while the D354G substitution in BR07-NA did not show any apparent impact on the Km values. The results together suggest that the 4 substitutions in the BR07-NA reduced the NA activity by two different mechanisms, and only the Q222R played both roles.

BR07-NA resulted in a slightly reduced unit activity, which can be associated with its slightly reduced enzyme affinity. Furthermore, the Q222R substitution in the BR07-NA led to a remarkably low unit activity with either the absence or presence of the $\mathrm{H} 275 \mathrm{Y}$ mutation, so its dual role in protein expression and enzyme affinity explained the most drastic decrease of activity by this substitution. The M234V in the BR07-NA retained a similar level of unit activity, suggesting this substitution only reduced the protein expression, but not the enzyme affinity; both the E329K and N344D led to reduced unit activities to a greater magnitude with the presence of the $\mathrm{H} 275 \mathrm{Y}$ mutation. 
A



C

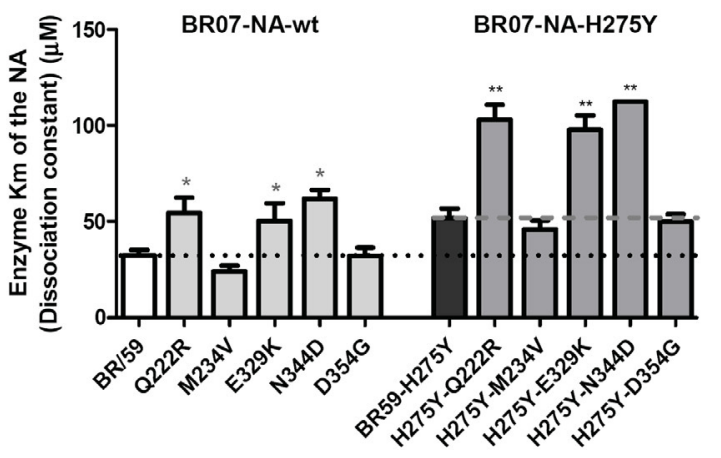

B

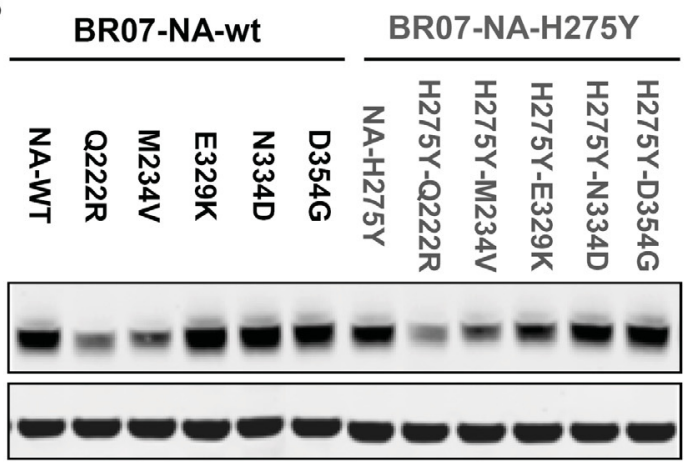

$\mathrm{D}$



Figure 3-10. Effects of five different single amino acid substitutions in the BR07-NA genes on NA enzyme activity, $\mathrm{km}$ value and protein expression.

(A) The enzyme activities of the variant BR07-NA proteins with 5 different single amino acid substitutions with the absence or presence of the $\mathrm{H} 275 \mathrm{Y}$ mutation respectively. Each substitution is labeled below the graph. (B) The detection of the same variant BR07-NA proteins of the 5 single amino acid substitutions by western blot. The upper panel is the detection by anti-HA tag antibodies, and the lower is by anti-Actin antibodies. (C) The NA enzyme $\mathrm{Km}$ value of the $\mathrm{rgBR} / 59 / 07$ viruses of BR07-NA variants of the 5 single substitutions by enzyme kinetics assay. (D) The enzyme activity per unit of protein (random unit of gray-scale of protein band) of the variant BR07-NA proteins of the 5 single substitutions. The data were calculated by using the enzyme activity of each NA variant divided by the protein quantity (measured as the density of the protein bands), and then the results were relative to the BR07-NA-wt. In all graphs, all values are the mean of 3 determinations $\pm \mathrm{SD} . * * *<0.05$ by $\mathrm{t}$ test when compared to $\mathrm{BR} / 59$ or $\mathrm{BR} / 59-\mathrm{H} 275 \mathrm{Y}$ respectively. 
The D354G in the BR07-NA was the only substitution increasing the unit activity, consistent with the increased enzyme activity above, but apparently not through the mechanism of enzyme affinity.

In conclusion, by using a loss-of-activity strategy in the BR07-NA, we discovered that in the genetic background of the BR07-like NAs, one group of molecular markers (Q222 and M234 residues) functioned to keep the high level of NA protein expression, and another group of molecular markers (Q222, K249 and N344 residues) served to provide the high level of NA enzyme affinity to substrate binding. The two groups of markers not only maintained the high quantity and high quality of the BR07-like NAs, and more importantly, they also essentially counteracted the negative effects of the $\mathrm{H} 275 \mathrm{Y}$ mutation on the protein expression and enzyme affinity, thus conferring the $\mathrm{H} 275 \mathrm{Y}$ tolerance to the NA protein. In addition, the D354G substitution in the BR07-NA somehow increased the enzyme activity even with the presence of the H275Y mutation, by unidentified mechanism.

\section{Effects of the Reciprocal Single Amino Acid Substitutions in GA06-NA Genes (from NC99-like to BR07-like) on Protein Function and Expression}

Now that we identified 4 amino acid residues (Q222, M234, K249, N344) in the BR07-NA responsible for its high NA quantity and quality, we proceeded to tested if the reciprocal amino acid substitutions (from NC99-like to BR07-like) at the 4 positions in NC99-like NAs were able to restore the low quantity and quality of NC99-like NAs to the high level of BR07-like NAs. We substituted each of the 4 amino acid residues in the GA06-NA gene (NC99-like lineage) with the corresponding BR07-like consensus residues respectively, as R222Q, V234M, E329K and D344N, with the absence or the presence of the $\mathrm{H} 275 \mathrm{Y}$ mutation. Then we generated the reverse-genetics viruses by using the 8 variants of the GA06-NA genes and compared them with the GA06-NA and BR07-NA derived viruses as described above. The GA06-NA naturally has G residue at the 354 position, so the D354G reversion was not counted into the analysis. The impact of each of the 4 substitutions in the GA06-NA gene on virus plaque size, enzyme activity, protein expression and enzyme affinity were examined and compared.

As for the plaque size of the reverse-genetic viruses (Fig 3-11A, Fig 3-12), the GA06-NA gene generated pinpoint-like plaques owning to its low activities $(0.5 \mathrm{~mm})$ as showed above, and the H275Y mutation in the GA06-NA caused a further reduction of the inherently small plaque size $(0.3 \mathrm{~mm})$. Each of the 4 substitutions in the GA06-NA increased the virus plaque size respectively but to different magnitudes: the R222Q or E329K alone significantly increased virus plaque size $(1.3$ and $2.0 \mathrm{~mm}$ respectively, $\mathrm{p}<0.05)$ but to a slightly smaller degree with the presence of the H275Y mutation (1.1 and $1.8 \mathrm{~mm}$ respectively). The V234M or D344N alone in the BR07-NA did not greatly change the virus plaque size ( 0.6 and $0.6 \mathrm{~mm}$ respectively), but with the presence of the $\mathrm{H} 275 \mathrm{Y}$ mutation, both substitutions effectively prevented the further plaque size reduction by the $\mathrm{H} 275 \mathrm{Y}$ mutation ( 0.6 and $0.5 \mathrm{~mm}$ respectively). 



Figure 3-11. Effects of four different single amino acid substitutions in the GA06NA genes on plaque size of reverse-genetics viruses, NA enzyme activity, NA Km value and NA protein expression.

(A) The plaque sizes of the $\mathrm{rgBR} / 59 / 07$ viruses by using 10 variants of GA06-NA genes with 4 different single amino acid substitutions at the absence or presence of the H275Y mutation respectively. The enzyme activities $(B)$ and protein detections $(C)$ of the variant GA06-NA proteins with the 4 single substitutions with the absence or presence of the $\mathrm{H} 275 \mathrm{Y}$ mutation respectively. (C) The upper panel is the detection by anti-HA tag antibodies, and the lower is by anti-Actin antibodies. (D) The NA enzyme Km value of the $\mathrm{rgBR} / 59 / 07$ viruses of GA06-NA variants of the 4 single substitutions by enzyme kinetics assay. In all graphs, all values are the mean of 3 determinations $\pm \mathrm{SD}$. $*$, ** $\mathrm{P}<0.05$ by $\mathrm{t}$ test when compared to GA06-wt or GA06-H275Y respectively. 
Wild-type sensitive viruses

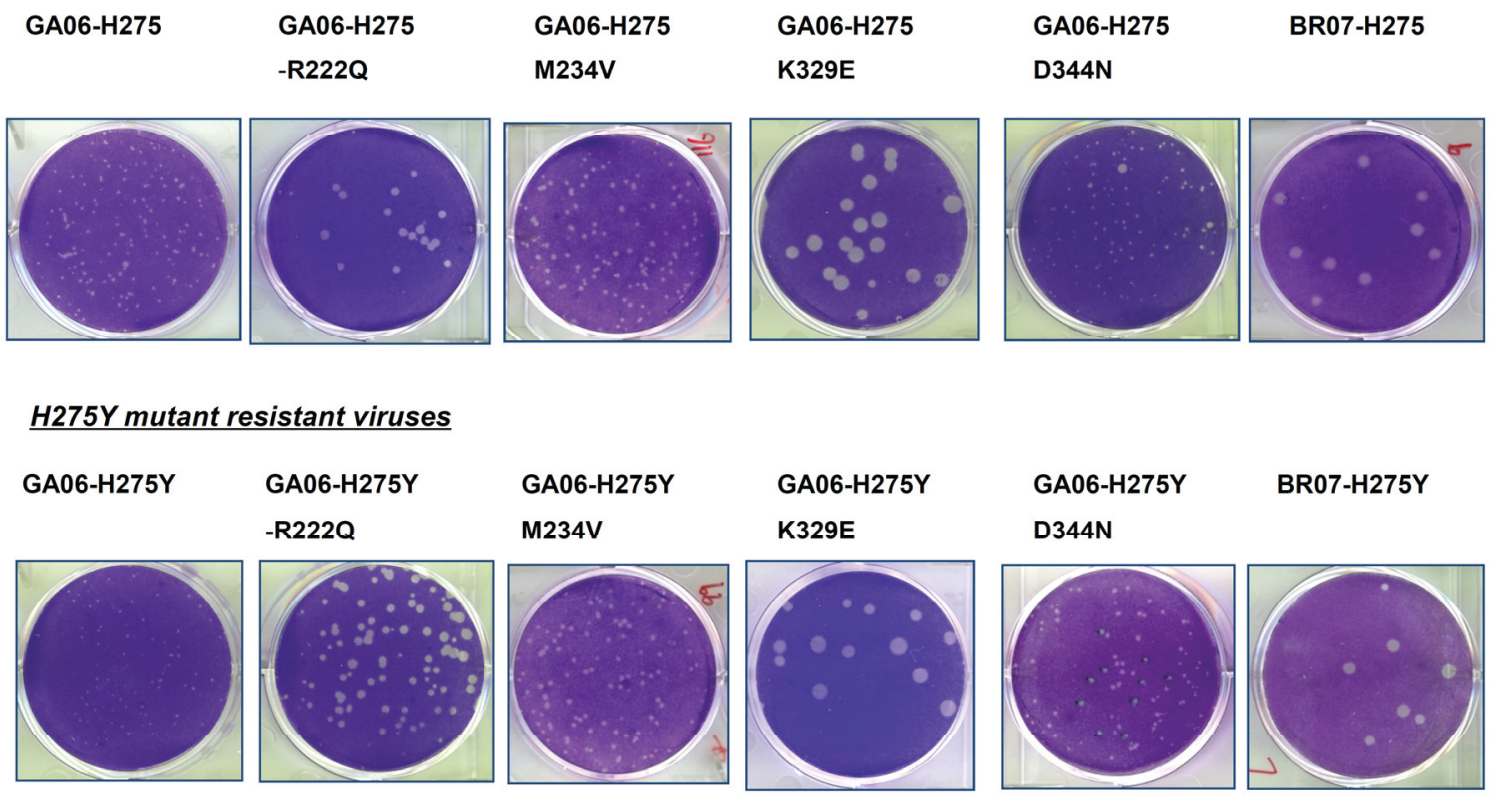

Figure 3-12. The plaque morphology of rgBR/59/07 viruses by using variant GA06NA genes with different single amino acid substitutions.

The plaque morphologies of the rgBR/59/07 viruses by using 10 variants of GA06-NA gene with 4 different single amino acid substitutions at the absence or presence of the $\mathrm{H} 275 \mathrm{Y}$ mutation respectively. The mutation sites were shown above each image. 
Regarding the enzyme activity of the GA06-NA variants of the single amino acid substitutions (Fig 3-11B), the H275Y mutation in the GA06-NA further decreased its intrinsically-low enzyme activity, as shown above. The R222Q or E329K substitution alone in the GA06-NA significantly increased enzyme activities $(\mathrm{p}<0.05)$, although to a smaller degree with the presence of the H275Y mutation. The V234M or D344N substitution alone in the GA06-NA slightly increased the enzyme activities, but at the presence of the $\mathrm{H} 275 \mathrm{Y}$ mutation, both substitutions essentially alleviated the loss of the activity caused by $\mathrm{H} 275 \mathrm{Y}$ mutation.

We examined if the 4 amino acid substitutions in the GA06-NA increase the enzyme activity by the mechanisms we identified above and we firstly determined their effects on total protein expression level of the GA06-NA (Fig 3-11C). As shown above (Fig 3-3B), the protein expression level of GA06-NA was naturally-low, and the H275Y mutation in the GA06-NA aggravated the low expression level to an even lower level. Both R222Q and V234M substitution alone in the GA06-NA greatly increased the protein expression levels, even with the presence of the $\mathrm{H} 275 \mathrm{Y}$ mutation. The E329K and D344N substitutions in the GA06-NA did not show any evident effect on protein expression. The results on protein expression were consistent with the roles of the 4 markers we identified in the BR07-NA as above.

We also tested the effects of the 4 substitutions in the GA06-NA on the enzyme $\mathrm{Km}$ value (Fig 3-11D). The Km value of the GA06-NA was intrinsically high as shown above (Fig 3-3D), and the H275Y mutation in the GA06-NA significantly raised the inherently high $\mathrm{Km}$ value to a higher degree $(\mathrm{p}<0.05)$. The R222Q, E329K and D344N substitutions alone in the GA06-NA significantly lowered the Km values respectively $(p<0.05)$, but to a smaller degree with the presence of the H275Y mutation. The V234M substitution in GA06-NA did not show any impact on the Km value. The results on the enzyme Km value were consistent with roles of the 4 markers we identified in the BR07-NA as above.

In conclusion, by using a reciprocal substitution strategy in the GA06-NA, we found that in the genetic background of the NC99-like NAs, the R222 and V234 two residues were responsible for the low level of NA protein expression, and the R222, E329 and D344 three residues accounted for the low NA enzyme affinity to substrate binding. Furthermore, the NC99-like NAs were not only inherently low in quantity and quality due to the two groups of molecular markers, they also could not counteract the additional diminishing effects of the $\mathrm{H} 275 \mathrm{Y}$ mutation on the protein expression and enzyme affinity and thus had no $\mathrm{H} 275 \mathrm{Y}$ tolerance. On the other hand, each of the four reciprocal substitutions in the NC99-like NA partially but significantly increased the enzyme activity by increasing the protein quantity and/or quality. More importantly, the improved NA quantity and quality by the 4 reciprocal substitutions in the NC99-like NA partially counteracted the deleterious effects of the H275Y mutation on protein expression and enzyme affinity, and conferred at least partial degree of tolerance to the H275Y mutation. 


\section{Determination of the Timeline of Fixations of the Four Molecular Markers during Evolution of N1 NA Genes}

Now that we have identified that each of the 4 amino acid substitution (R222Q, V234M, K329E, D344N) in the NC99-like NA can partially but significantly improve the NA protein quantity and quality, we then sought to uncover the temporal sequence of the fixation of the four molecular markers into the NA gene during seasonal N1 evolution, and to understand if the sequential addition of all four molecular makers could fully reconstruct the quantity and quality of the NC99-like NAs to the level equivalent to those of the BR07-like NAs.

The phylogenetic analysis with incorporation of a temporal scale and a probabilistic analysis were used to determine the relative timeline of the fixation of each of the 4 molecular markers (Fig 3-13). The 354 position is of special interest for its reversion between the two lineages, so it was also included along with the other four positions for the analysis. First of all, the H275Y mutation appeared in a sporadic and random fashion before 2007. Since then, the H275Y mutation was fixed into the majority of the N1 populations especially during 2008 2009. Secondly, remarkably, all four amino acid substitutions emerged and were fixed into the N1 population prior to the fixation of the $\mathrm{H} 275 \mathrm{Y}$ mutation; The 4 amino acid substitutions were fixed into the NA gene in a sequential manner in the order of V234M - R222Q - D344N - K329E. Last, puzzlingly, a G354D substitution firstly occurred during the NA divergence at 2005, but later a D354G reversion arose, coincident with the fixation of H275Y mutation at 2008. Taken together, the timeline analysis is consistent with the previous lineage consensus residue analysis, but it is more informative as it provides evidence and insights about when the naturally-occurring oseltamivir-resistant H1N1 viruses emerged and how their emergence was associated with acquisition of the $\mathrm{H} 275 \mathrm{Y}$ tolerance by the 4 molecular markers.

\section{Effects of Sequential Addition of the Four Molecular Markers into GA06-NA Genes on Protein Function and Expression}

To simulate the sequential fixation of 4 molecular markers during the NA evolution history, we sequentially placed the 4 amino acid substitutions into the GA06-NA gene one by one in an order indicated by the timeline analysis (V234M R222Q - D344N - K329E), with the absence or presence of the H275Y mutation. Then we generated the reverse genetics viruses by using the 6 variants of the GA06-NA gene and compared them with GA06-NA and BR07-NA derived viruses as described above. As each of the four substitutions were added into GA06-NA sequentially, the virus plaque size and the enzyme activity increased accordingly in a progressive way (Fig 3-14A, B, Fig 3-15), eventually to the degree fully equivalent to or even slightly higher than those of BR07-NA when the fourth substitution was added. With the presence of the $\mathrm{H} 275 \mathrm{Y}$ mutation, the sequential addition of each substitution lead to the similar progressive increase of plaque size and enzyme activity as that in the absence of the $\mathrm{H} 275 \mathrm{Y}$ mutation, but at a slightly small magnitude. More importantly, the sequential 




Figure 3-13. The timeline of fixation of the 6 amino acid residue substitutions in the seasonal N1 NA genes.

NA genes of representative 249 human seasonal H1N1 viruses were used for phylogenetic analysis. The tree was constructed by using the Bayesian simulations method. The probabilistic model was used to analyze of amino acid fixation by ancestral state reconstruction. The trunk color change shows that the average population possesses that particular amino acid residue indicated by the color code. Each tree is the same but shows the fixation of the amino acid residues for each of the 6 amino acid positions of NA proteins. The upper and lower arrow shows the time when the respective V234M and $\mathrm{H} 275 \mathrm{Y}$ were fixed into the population. 
A

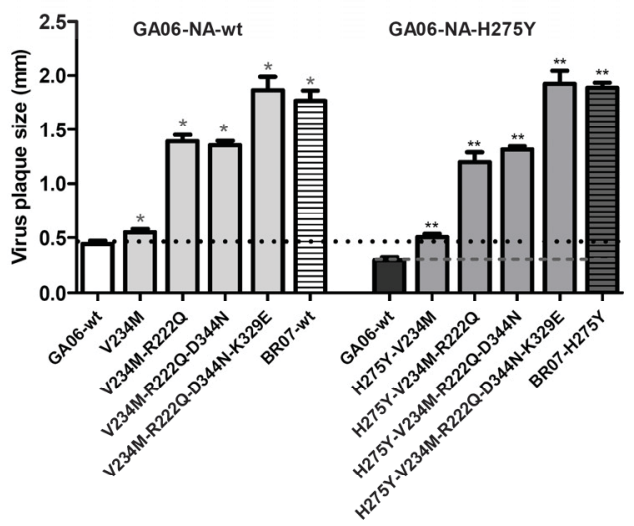

$\mathrm{C}$

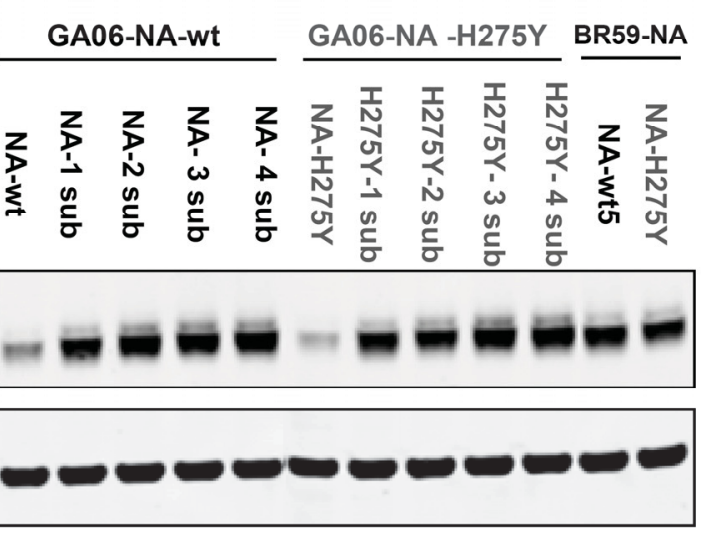

$\mathrm{B}$
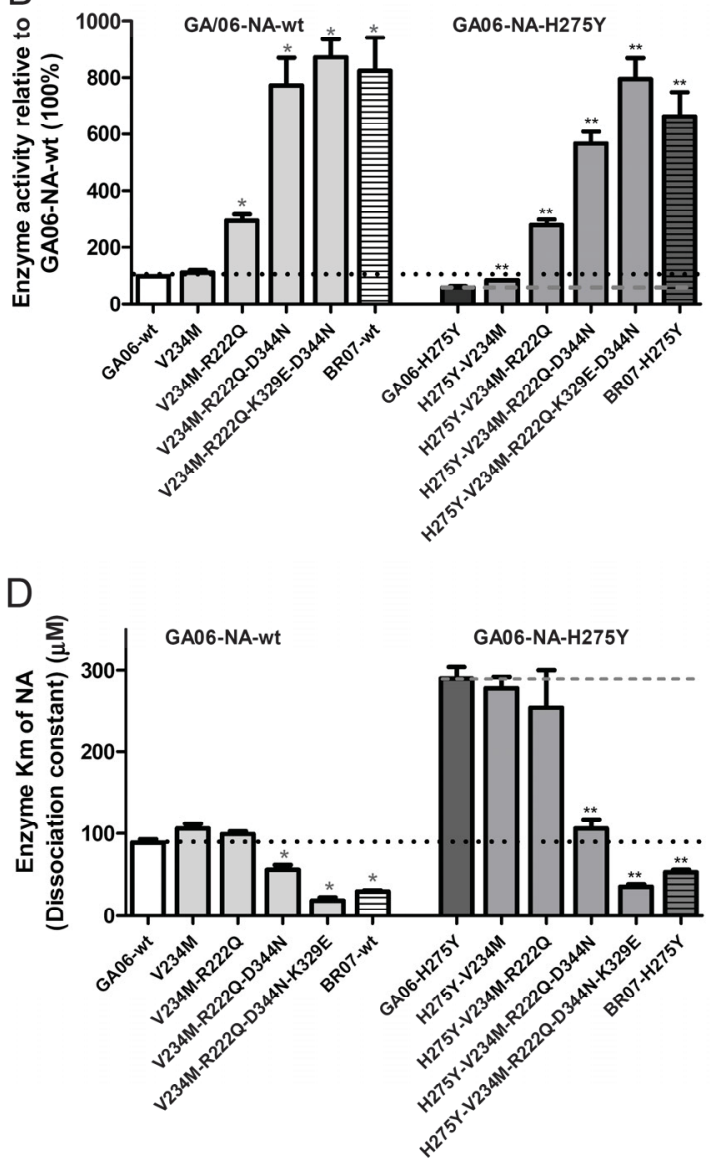

Figure 3-14. Effects of sequential addition of four amino acid substitutions in the GA06-NA genes on plaque size of reverse-genetics viruses, NA enzyme activity, NA Km value and NA protein expression.

(A) The plaque sizes of the rgBR/59/07 viruses by using 10 variants of GA06-NA genes with sequential addition of 4 different single amino acid substitutions with the absence or presence of the $\mathrm{H} 275 \mathrm{Y}$ mutation respectively. The enzyme activities (B) and protein detection (C) of the 10 variant GA06-NA proteins with the sequential addition of 4 substitutions with the absence or presence of the H275Y mutation respectively. (C) The upper panel is the detection by anti-HA tag antibodies, and the lower is by anti-Actin antibodies. (D) The NA enzyme Km value of the rgBR/59/07 viruses of GA06-NA variants with the sequential addition of the 4 substitutions by enzyme kinetics assay. The order of the sequential addition is as V234M + R222Q + D344N + K329E. In all graphs, all values are the mean of 3 determinations $\pm \mathrm{SD} . * * * \mathrm{P}<0.05$ by $\mathrm{t}$ test when compared to GA06-wt or GA06-H275Y respectively. 
Wild-type sensitive viruses

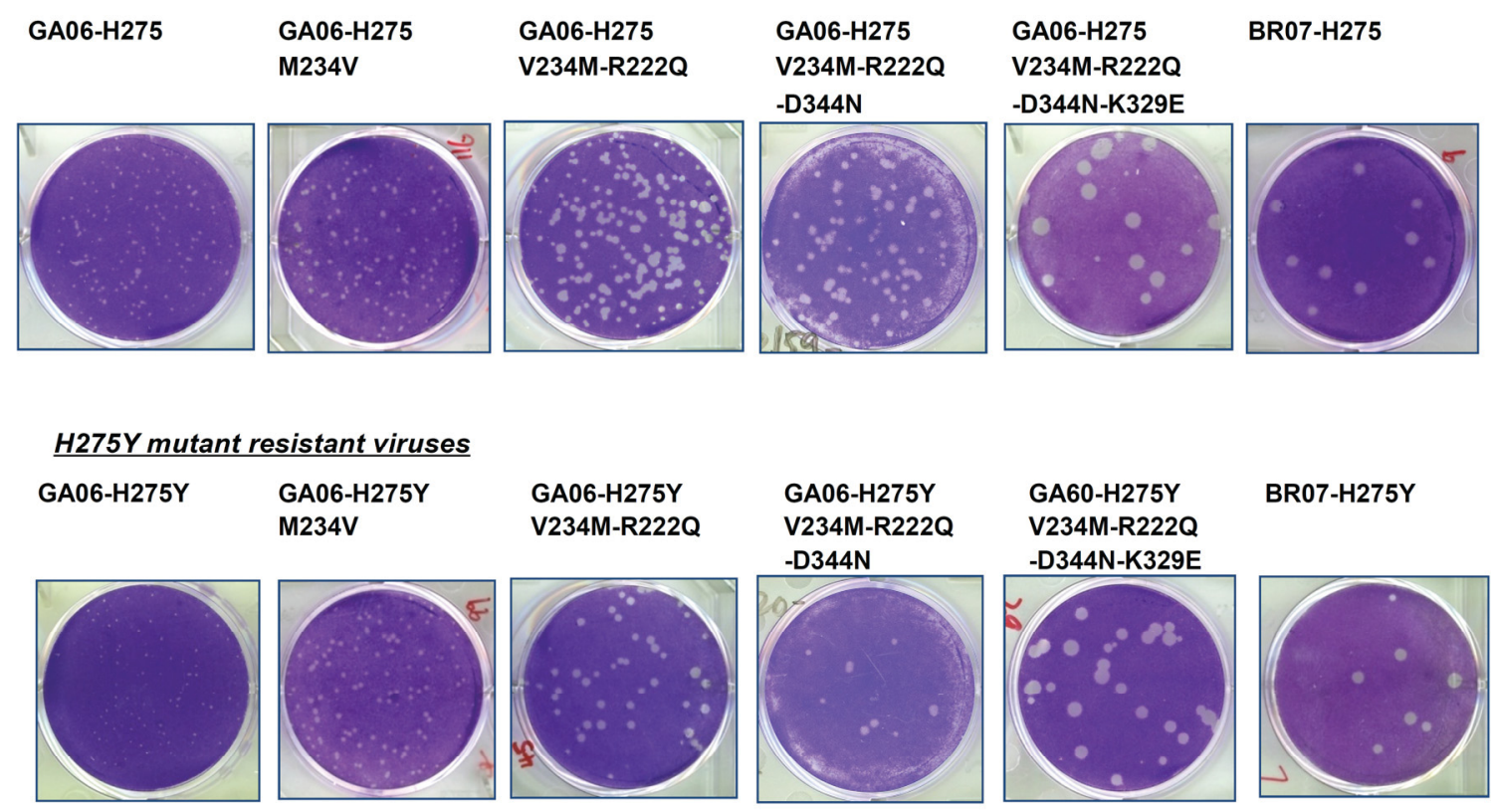

Figure 3-15. The plaque morphologies of $\mathrm{rgBR} / 59 / 07$ viruses by using variant GA06-NA genes with sequential addition of the 4 amino acid substitutions.

The plaque morphologies of the rgBR/59/07 viruses by using 10 variants of GA06-NA gene with 4 different single amino acid substitutions at the absence or presence of the $\mathrm{H} 275 \mathrm{Y}$ mutation respectively. 
addition at each step alleviated the degree of the loss of activity caused by the $\mathrm{H} 275 \mathrm{Y}$ mutation, and the addition of all four substitutions into GA06-NA eventually led to only a marginal loss of the activity by $\mathrm{H} 275 \mathrm{Y}$ mutation, equivalent to the minimal degree of activity loss in the BR07-NA by the H275Y mutation.

We then examined the effects of the sequential addition of 4 amino acid substitutions in the GA06-NA on the protein expression and enzyme affinity (Fig 3-14C, D). The addition of the first two substitutions V234M and R222D into the GA06-NA not only greatly increased the level of NA protein expression to that of BR07-NA, but also effectively prevented the reduction of protein expression caused by the H275Y mutation. The addition of the next two substitutions V344D and E329K didn't show any further impact on the protein expression. The first addition of V234M into the GA06-NA didn't cause a measurable change of the enzyme $\mathrm{Km}$ value, but the following addition of R222D, V344D and E329K progressively and significantly reduced the enzyme Km value ( $p<0.05$ ), eventually to a level equivalent to that of BR07-NA. Moreover, the sequential addition of the three substitutions also largely counteracted the diminishing effect by the H275Y mutation on enzyme affinity, and eventually controlled the level of enzyme affinity comparable to that of BR07-NA with the presence of the H275Y mutation.

In summary, the sequential addition of the 4 amino acid substitutions (V234M R222Q - D344N - K329E) greatly increased the NA protein quantity and then significantly increased the NA enzyme quality. There are 10 amino acid substitutions differentiating between the genotype of NC99-like and BR07-like NAs, but sequential addition of the 4 substitutions into the NC99-like NA was sufficient to reconstruct the NA protein quantity and quality to a level equivalent to those of the BR07-like NA. More importantly, the high NA protein quantity and quality by the 4 substitutions effectively counteracted the diminishing effects of the H275Y mutation on the protein expression and enzyme affinity, leading to only a marginal loss of the enzyme activity by the H275Y mutation, thus successfully conferring tolerance to the $\mathrm{H} 275 \mathrm{Y}$ mutation.

\section{Determination of NA Tolerance to the H275Y Mutation in 2009 Pandemic H1N1 Viruses}

In our previous study in Chapter 2, we characterized the transmissibility, growth, and NA kinetics of a wild-type (DM/524/09) and a H275Y mutant (DM/528/09) 2009 pandemic $\mathrm{H} 1 \mathrm{~N} 1$ viruses ( $\mathrm{pH} 1 \mathrm{~N} 1)$, which only differed at the H275Y mutation. We found that the $\mathrm{H} 275 \mathrm{Y}$ mutant $\mathrm{pH} 1 \mathrm{~N} 1$ virus had lower transmissibility in ferrets, delayed growth in cells and reduced NA affinity in vitro than the wild-type $\mathrm{pH} 1 \mathrm{~N} 1$, so we concluded that the $\mathrm{H} 275 \mathrm{Y}$ mutant $\mathrm{pH} 1 \mathrm{~N} 1$ virus had lower fitness relative to the wild-type $\mathrm{pH} 1 \mathrm{~N} 1$ virus. In the present study, we identified that the NA tolerance to the $\mathrm{H} 275 \mathrm{Y}$ mutation largely determined the virus fitness level of H275Y mutant viruses in the seasonal H1N1 virus lineage. Next, we sought to evaluate if and how the attenuated virus fitness in the $\mathrm{H} 275 \mathrm{Y}$ mutant $\mathrm{pH} 1 \mathrm{~N} 1$ virus was associated with the NA H275Y intolerance. 
NA gene comparison between the pandemic and seasonal H1N1 viruses. We first examined and compared the consensus sequences of NA genes between seasonal and 2009 pandemic H1N1 viruses, specifically with a focus at the four amino acid positions with permissive residues for the H275Y mutation (Table $3-2$ ). There is $\sim 25 \%$ dissimilarity in amino acid residues compositions of the NA genes between seasonal $\mathrm{H} 1 \mathrm{~N} 1$ and $\mathrm{pH} 1 \mathrm{~N} 1$ viruses, which is associated with their distant ancestor in N1 gene evolution (22). We only listed the NA amino acid residues of pH1N1 viruses at the 10 positions at which the residues differentiate the NA genes of two seasonal N1 lineages, which also showed great diversity from the seasonal lineages. By far, no predominant difference was found in the NA genes between the wild-type and H275Y mutant $\mathrm{pH} 1 \mathrm{~N} 1$ viruses. With regards to the 4 amino acid positions permissive for the $\mathrm{H} 275 \mathrm{Y}$ mutation in seasonal N1 lineage, the NA genes of $\mathrm{pH} 1 \mathrm{~N} 1$ viruses showed both conservation and diversity at the 4 positions. The NAs of $\mathrm{pH} 1 \mathrm{~N} 1$ viruses similarly encode $\mathrm{V}$ residue at the 234 position as the NC99-like NAs, which was linked to the inherently-low NA protein expression. pH1N1 NAs encode $\mathrm{N}$ residue at the 344 site same as BR07-like NAs, which was linked the inherently-high NA enzyme affinity; but pH1N1 NAs encode a different $\mathrm{N}$ residue at 222 position other than the R or Q in seasonal NAs, and N residue at 329 position other than the $\mathrm{E}$ or $\mathrm{K}$ in seasonal NAs. Considering the extensive diversity in the NA amino acid residue compositions between $\mathrm{pH} 1 \mathrm{~N} 1$ and seasonal H1N1 lineage, further extensive investigation is needed to characterize what roles these distinct molecular markers have in determining the NA protein quantity and quality respectively.

NA activity, protein expression and enzyme affinity of the wild-type and H275Y mutant pandemic H1N1 viruses in vitro. Next, we evaluated if the NA protein of the $\mathrm{pH} 1 \mathrm{~N} 1$ virus have the tolerance to the H275Y mutation or not in the level of NA enzyme activity, protein expression level and enzyme affinity, in spite of the diverse NA amino acid residues. Two pairs of NA proteins, the wild-type and H275Y mutant of BR07-NA and GA06-NA were compared with the NAs of DM/524/09 and DM/528/09 viruses (DM09-NA) respectively regarding to NA tolerance to the H275Y mutation.

In the level of NA enzyme activity (Fig 3-16A), we compared the percentage loss of enzyme activity in each $\mathrm{H} 275 \mathrm{Y}$ mutant NA proteins relative to its respective matching wild-type NA. The results showed the H275Y mutation caused loss of activity in the NA proteins of all three lineages, with lowest magnitude in the BR07-NA $(\sim 20 \%)$, and the greatest magnitude $(\sim 70 \%)$ in the DM09-NA which was even bigger than that in the GA06-NA $(\sim 50 \%)$. The results suggested that the DM09-NA was not able to tolerate the negative effects of the $\mathrm{H} 275 \mathrm{Y}$ mutation on enzyme activity, with a huge loss of the activity in the H275Y mutant of DM09-NA.

As for the NA protein expression (Fig 3-16B), the results showed that the protein expression level of the wild-type DM09-NA was inherently lower than that of the wild-type BR07-NA, and similar to that of the GA06-NA. The H275Y mutation further greatly reduced the inherently-low level of total protein expression of the DM09-NA and the GA06-NA, but hardly changed the level of the BR07-NA. The flowcytomerty confirmed that the cell surface accumulation of the DM09-NA was also greatly diminished by the H275Y mutation (Fig 3-16C). The results suggested that the 
A

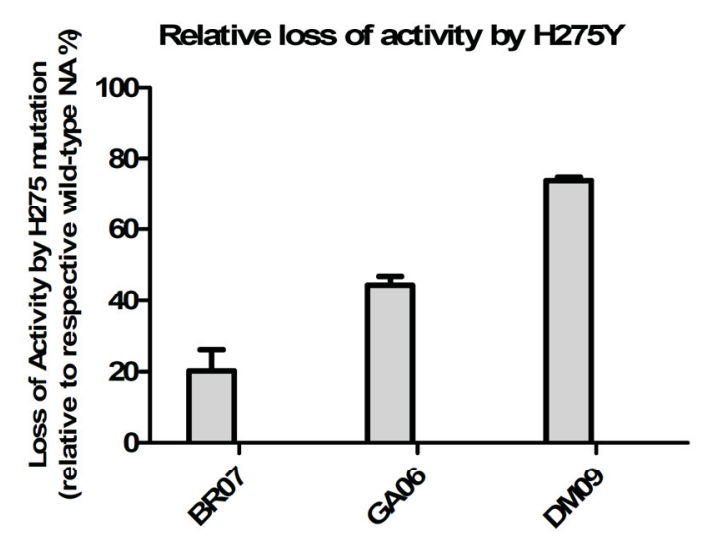

C

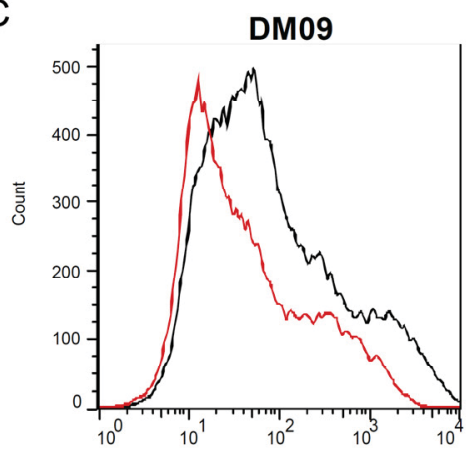

B


Figure 3-16. The NA activities, protein expression and enzyme Km values of the 2009 pandemic H1N1 viruses.

(A) The percentage loss of enzyme activities of the H275Y mutant NA of the DM09 pandemic $\mathrm{H} 1 \mathrm{~N} 1$ virus relative to its wild-type NA, with comparison to the loss of activity in BR07 and GA06 NA protein pairs. (B) The detection protein expression of the wild-type and $\mathrm{H} 275 \mathrm{Y}$ mutant of DM09-NA proteins, with comparison the loss of activity in BR07 and GA06 NA protein pairs. The upper panel is the detection by anti-HA tag antibodies, and the lower is by anti-Actin antibodies. (C) The surface protein accumulation of DM09-NA proteins by flowcytometry. Black line indicates the wild-type protein and red line indicates the $\mathrm{H} 275 \mathrm{Y}$ mutant protein. 
DM09-NA was not able to tolerate the diminishing effects of the H275Y mutation on NA protein expression, with greatly reduced protein expression of the $\mathrm{H} 275 \mathrm{Y}$ mutant of DM09-NA.

As regards to NA enzyme affinity to substrate binding, a retrospective examination of the data in the previous and present studies (Table 2-1, Fig 3-7D) showed the wild-type DM09-NA exhibited a slightly higher Km value than the wild-type BR07-NA, suggesting its slightly lower enzyme affinity of substrate binding. The H275Y mutation further increased Km value of the DM/09-NA with a bigger magnitude than that of BR07-NA, but a smaller magnitude than that of GA06-NA. The results suggested that the DM09-NA had naturally relatively low enzyme affinity and the H275Y mutation further reduced its enzyme affinity. Although it is hard to determine if the DM09-NA had the tolerance to $\mathrm{H} 275 \mathrm{Y}$ mutation on enzyme affinity level or not, as the reduction of the affinity was intermediate between the BR07-NA and GA06-NA, the H275Y mutation still had a negative effect on the enzyme affinity of the DM09-NA.

In summary, the significant loss of the enzyme activity in the H275Y NA of the $\mathrm{pH} 1 \mathrm{~N} 1$ virus was caused by greatly reduced NA protein expression as well as reduced NA enzyme affinity by the H275Y mutation, which suggested the NA of $\mathrm{pH} 1 \mathrm{~N} 1$ virus now had no tolerance to the H275Y mutation. The NA intolerance to the H275Y mutation on the NA protein level correlated well with the lower virus fitness of the $\mathrm{H} 275 \mathrm{Y}$ mutant $\mathrm{pH} 1 \mathrm{~N} 1$ virus than that of the wild-type $\mathrm{pH} 1 \mathrm{~N} 1$ virus, and further explained the delayed growth in cells and the transmissibility of the H275Y pH1N1 virus in ferrets.

\section{Discussion}

About the unorthodox naturally-occurring osletamivir-resistant seasonal H1N1 viruses, one of biggest questions remained is what fundamental changes occurred in the NA genes and consequently in the NA proteins of the H1N1 viruses over the first decade of oseltamivir use. The findings of two permissive NA mutations enabling the evolution of $\mathrm{H} 275 \mathrm{Y}$ mutant viruses significantly advanced our understanding of the defects caused by the H275Y mutation on the NA proteins, but it still left the question pending about what was the intrinsic changes or evolvement in the NA genes and proteins of the seasonal H1N1 viruses other than the defects caused by the H275Y mutation. This study, to our knowledge, is the first to understand how the genetic and phenotypic divergence of the NA proteins by themselves can allow the fixation of the H275Y mutation into the NA proteins during the NA evolution history, apart from understanding the defects caused by the H275Y mutation, which is underlying NA molecular basis of the naturally-occurring oseltamivir-resistant $\mathrm{H} 1 \mathrm{~N} 1$ viruses.

By integrating NA genotype evolution with the consequent NA protein phenotype evolvement, we uncovered the at least some monumental "scenes" when the fundamental changes in NA genes and proteins occurred during the evolution of the seasonal H1N1 viruses : 1) in the earlier years between 1999 2006, a lineage of human H1N1 viruses 
called NC99-like viruses were the predominant circulating. The NC99-like NA proteins were inherently low in quantity and quality (protein expression and enzyme affinity), and the presence of the H275Y NA mutation could further diminish the inherently-low quantity and quality, which was destructive for the NA function. As the NC99-NAs cannot tolerate the presence of $\mathrm{H} 275 \mathrm{Y}$ mutation on protein level, the $\mathrm{H} 275 \mathrm{Y}$ mutant viruses had lower virus fitness level than the wild-type viruses. Owning to this reason, during that time, the NC99-like H275Y mutant viruses occasionally emerged under drug selection pressure, but the $\mathrm{H} 275 \mathrm{Y}$ mutant viruses were soon extinguished by the wild-type viruses once the drug selection pressure was removed. 2) During the continuing H1N1 virus evolution, the NC99-like H1N1 viruses were gradually acquiring amino acid substitutions in NA genes as well as in HA genes. Starting from around 2003, the NC99-like NA genes sequentially acquired V234M and R222D substitution, which greatly enhanced the NA protein expression level; following that, the NA genes subsequently accumulated V344D and E329K substitutions, which significantly increased the NA enzyme affinity to substrate binding. 3) After the years, the sequential addition of a total of ten amino acid substitutions including the four function-altering substitutions into the NC99-like NA genes eventually led to emergence of a new lineage of NA genes and corresponding viruses at 2007, the BR07-like H1N1 viruses. The BR07-like NA proteins were naturally high in protein quantity and enzyme quality as a result of the four amino acid substitutions; more importantly, the high NA quantity and quality were able to counteract the negative effects of the $\mathrm{H} 275 \mathrm{Y}$ mutation on the protein expression and enzyme affinity, and the function level of the BR07-like NA proteins was largely preserved with only a marginal loss; as the BR07-like NA proteins can tolerate the presence of the H275Y mutation on the protein level, the BR07-like H275Y mutant viruses retained a virus fitness level comparable to the wild-type viruses; thus they were able to continue circulation with wild-type viruses in the absence of the drug selection pressure until 2009 season before swine-origin pandemic influenza viruses took over the world.

In our opinion, we stress the NA genetic divergence from NC99-like into BR07-like H1N1 viruses including the acquisition of the 4 molecular markers in the first place was associated with NA protein antigenic drift. Among the 10 positions at which the amino acid differentiates the two NA lineages, 3 positions $(222,249,344)$ correspond to antibody recognition sites in the N2 NA protein (130-132) and the mutations at these three positions (Q222R, 249G, N344D) in the NA of BR/59/07 virus affected the reactivity of the different anti-sera to the virus in NA inhibition assay (133). The 329 position was also suggested as a potential subdominant conserved epitope because E329K mutation in the NA of BR/59/07 virus was very important for the NA protein recognition by the human sera (133). It is uncertain if other 6 positions are also involved in antigenic sites and further understanding of N1 NA protein antigenic sites is needed. Nevertheless, among the 4 molecular markers we identified, three of them $(222,344$, 329) has been showed to be involved in NA antigenic determinants. In the NA molecular structure, all these three positions are at the surface of NA globular head distant from the NA active site, and they are all at the opposite side to the tetramer interface (Fig 3-2) (133). Theoretically, the structure locations of these three positions potentially allow their access to antibody or substrate binding. We are uncertain how to connect the 
simultaneous changes in both NA antigencity and NA enzyme activity caused by the same amino acid residues in molecular level, and precise NA structures with different residue substitutions are required for answers to this question.

It should be stressed that the natural force driving the divergence of NA genes of the H1N1 viruses most likely is the host immunity against NA protein. The host immunity can target the virus surface protein HA and NA during infection and herd immunity against the two proteins accumulates over a time of circulation of the viruses. To evade the herd immunity against the proteins, both HA and NA genes constantly evolves by acquiring mutations at the antigenic sites, known as antigenic drift (134). On the other hand, an intriguing hypothesis about NA evolution is that NA genes may co-evolve with HA genes (27). HA proteins may be subject to more host immunity pressure than NA proteins, as HA protein binds to the sialic acid-containing receptors and neutralization of HA proteins by host immunity can annul the virus replication in hosts. Considering the receptor-binding activity of $\mathrm{HA}$ and opposite receptor-destroying activity of NA, the fast evolving HA genes may necessitate co-evolution of NA genes to acquire an optional HA-NA balance for efficient virus replication and transmission. Some studies have showed that the phylogeny of the HA genes exhibited the same cluster pattern as the NA genes in the seasonal H1N1 viruses (63-65). The matched cluster patterns of the HA and NA genes may reflect the paralleled co-evolution of the two genes and matched function levels of the two proteins in the viruses. Nevertheless, the NA divergence of NC99-like to BR07-like firstly is associated with antigenic drift, and the genetic changes of the NA gene may result in potential changes in other protein phenotypes, such as protein expression and function identified in our case.

It also should be stressed that the accumulation of the four NA molecular markers during the natural NA gene divergence from NC99-like into BR07-like is most likely just fortuitously beneficial for the $\mathrm{H} 275 \mathrm{Y}$ mutation. The four molecular markers can determine the low or high quantity and quality of NA proteins of the two lineages, but the terms of "low" or "high" are only relative between the two lineages. NA proteins neither being "low" nor "high" is a defect for the proteins to function as a part of whole infectious virus particle. In the present study, the wild-type NC99-like virus and BR07-like virus the virus exhibited the equivalent growth and transmissibility in the naive ferrets, which suggested that NC99-like viruses were not inferior to BR07-like viruses in the naïve hosts in terms of infectivity and transmissibility in spite of their "low" NA quantity and quality, and vice versa. The sustained circulation of the NC99-like viruses from 1999 2006 also suggests that the "low" NA quantity and quality is not a defect for the viruses themselves. The extinguishment of the NC99-like viruses by replacement of the BR07-like viruses primarily was the result of antigenic drift due to the accumulated herd host immunity against the NC99-like viruses over the extended circulation. BR07-like NA proteins being "high" quantity and quality are only fortuitously beneficial or "permissive" for the tolerance of the H275Y mutation. Only when the $\mathrm{H} 275 \mathrm{Y}$ mutations were introduced into the NA protein, the H275Y mutation had differential effects on the NAs proteins, then the superiority of NA proteins regarding to the tolerance to the $\mathrm{H} 275 \mathrm{Y}$ mutations can be separated. The "low" quantity and quality of the NC99-like NAs cannot tolerate the H275Y mutation, but the "high" quantity and 
quality of the BR07-like NAs can tolerate the H275Y mutation. In other words, if the $\mathrm{H} 275 \mathrm{Y}$ mutation had never emerged, the quantity or quality of the NAs would not have made a difference for the infectivity and transmissibility of the viruses. Concisely, the four NA molecular markers we identified here are 1) the residues associated with NA protein antigenic drift, 2) the residues associated with NA function in terms of protein quantity and enzyme quality, and 3) the residues associated with the tolerance to the $\mathrm{H} 275 \mathrm{Y}$ mutation on NA proteins.

In addition to the four NA molecular markers as described above, the NA 354 position is of a unique genotype and functional role during the NA evolution of the H1N1 viruses. Regarding the genotype of the position, during the NA divergence from the NC99-like into the BR07-like lineage, the amino acid residues were firstly substituted as G354D; then within the BR07-like lineage, it was exactly reversed as D354G in the majority of the $\mathrm{H} 275 \mathrm{Y}$ mutant viruses, but remained unchanged as D in all the wild-type viruses within the lineage. As regards to its functional role, the D354G slightly increased the NA activity in the BR07-NA, which is exactly opposite to other four substitutions in the BR07-NA (from BR07-like to NC99-like) that all reduced the NA activity. Moreover, unlike the other four positions, the D354G somehow increased the NA activity but not through the change in protein expression or enzyme affinity. By our results, it remains unknown why the NC99-like NAs sequentially acquired the four substitutions to increase their NA activity, but then adopted G354D which apparently functioned opposite to NA activity. In spite of the enigmatic initial G354D substitution during the lineage switch, the later D354G reversion coinciding with $\mathrm{H} 275 \mathrm{Y}$ mutation within the BR07-lineage seemed to have further increased the enzyme activity of $\mathrm{H} 275 \mathrm{Y}$ mutant NAs and thus have repaired the marginal loss of NA activity caused by the $\mathrm{H} 275 \mathrm{Y}$ mutation, which suggests that the later D354G reversion is also a beneficial mutation for the NA function in the H275Y mutant viruses.

In the timeline of the fixation into NA genes, especially relative to the fixation of the $\mathrm{H} 275 \mathrm{Y}$ mutation, the $\mathrm{D} 354 \mathrm{G}$ reversion is of unique standing different from the other four molecular markers in N1 evolution history. In the timeline the four molecular markers arose sequentially between 2002 2006 during the NA divergence toward the BR07-like lineage, and the puzzling G354D substitution occurred around 2005 2006 at later stages of the divergence while the D354G reversion coincided or closely followed the fixation of $\mathrm{H} 275 \mathrm{Y}$ mutation within the BR07-like lineage at 2008. By the integration of their relative fixation time and the respective function of the five molecular makers, we are able to further differentiate the unique role of the D354G reversion from the other four molecular markers. From a point of view of the molecular evolution of multiple mutations $(67 ; 135)$, the later D354G reversion can be better described as a "compensatory" mutation, which followed the major functional mutation (the H275Y mutation in this case) and it might be selected to aim to repair the function damage caused by the major functional mutation. Whereas the other four molecular markers rather better be classified as "permissive" residues, which occurred initially prior to the major functional mutation (the $\mathrm{H} 275 \mathrm{Y}$ mutation), but were fortuitously beneficial for the major functional mutation. 
The NA tolerance to the H275Y mutation at the protein level conferred by both the "permissive" residues and the "compensatory" mutation had profound ramification on virus fitness level. It is worth noting that virus fitness describes its adaptation or relative ability to produce infectious progeny in a host (39). A key concept about virus fitness is "relative". Although originally a growth competition experiment between different viruses is used to define the fitness level of a virus relative to another virus (39), now, as an alterative, a comparative experiment between different viruses generally can be used to describe the fitness level of a virus relative to another virus. In our discussion, the "relative" is between wild-type and H275Y mutant viruses. The comparative virus infectivity and transmissibility etc all can demonstrate the virus fitness level relative to each other. In previous report at 2002, an $\mathrm{H} 275 \mathrm{Y}$ resistant variant exhibited severely compromised replication and virulence both in vitro and in vivo compared with the wild-type virus $(54 ; 55)$, suggesting compromised fitness of the $\mathrm{H} 275 \mathrm{Y}$ virus, presumably a NC99-like virus, relative to the wild-type virus. While in more recent studies, the replication of the recent BR07-like H275Y mutant viruses was not impaired in cells and ferrets compared to the wild-type viruses (61), suggesting uncompromised fitness of the viruses relative to the wild-type viruses. In our present study, we explicitly demonstrated that the replication and transmissibility of the NC99-like H275Y viruses in ferrets was significantly attenuated, while those of the BR07-like H275Y viruses were minimally altered, when compared with those of their counterpart wild-type viruses. The results clearly suggested the distinct fitness level of the H275Y mutant viruses relative to the corresponding wild-type viruses in the two lineages. The present study is closely correlated with the previous reports, which strongly supported that the NA intolerance or tolerance to the $\mathrm{H} 275 \mathrm{Y}$ mutation in protein level largely determined the fitness outcome of the $\mathrm{H} 275 \mathrm{Y}$ mutant viruses relative to the wild-type virus.

The viral fitness of $\mathrm{H} 275 \mathrm{Y}$ mutant viruses relative to wild-type viruses also can be illustrated by epidemiology data about their respective prevalence in circulation. The circulation prevalence of different viruses can be an ultimate manifestation of the virus fitness relative to each other in nature. Based on the surveillance studies of NA inhibitor susceptibility, between 1999 2006, there were extremely low prevalence of H275Y mutant influenza viruses among the globally collected influenza virus isolates (from $0 \sim 1.7 \%$ ) (32;46;48), suggesting that the H275Y mutant viruses had lower fitness advantage than the wild-type viruses and thus were extinguished from circulation at the time. However, between 2007 to 2009 pre-pandemic season, the H275Y mutant viruses were detected of global high prevalence in the context of absence of the drug treatment $(45 ; 56)$, even with a trend to override the wild-type viruses to become the dominant viruses, suggesting that the $\mathrm{H} 275 \mathrm{Y}$ mutant viruses had equivalent or even higher fitness advantage than the wild-type viruses and thus were sustained in circulation. Notably, the timeline of the contrasting prevalence level of $\mathrm{H} 275 \mathrm{Y}$ mutant viruses is also in accordance with the timeline of the circulation of NC99-like and BR07-like H1N1 viruses, which also in accordance with our findings that the distinct fitness level of $\mathrm{H} 275 \mathrm{Y}$ mutant viruses in the two lineages.

With the identification of the NA molecular basis including both "permissive" markers and "compensatory" mutations in the naturally-occurring H275Y resistant 
viruses, a retrospective look allows us to have a better understanding about the variations of the transmissibility of the H1N1 viruses in ferrets. In the BR07-like group, both HW/28/07 and rgBR/59/07-NA ${ }^{\mathrm{H} 275 \mathrm{Y}} \mathrm{H} 275 \mathrm{Y}$ mutant viruses exhibited slightly delayed direct-contact transmission but essentially uncompromised transmissibility compared to the wild-type virus. Now the delay can be correlated to a common feature in their genotypes that both viruses possessed all 4 permissive markers but lacked the compensatory mutation D354G in their NA protein. The NY/3467/09 virus H275Y mutant also exhibited slightly delayed direct-contact transmission and this virus had the lowest NA enzyme affinity among the tested BR07-like viruses. Now both phenotypes can be correlated to the absence of the permissive D344N in its NA protein, although it had acquired the 3 permissive markers and the compensatory mutation. Both the $\mathrm{NY} / 1672 / 09$ and $\mathrm{rgBR} / 59 / 07-\mathrm{NA}^{\mathrm{H} 275 \mathrm{Y}+\mathrm{D} 354 \mathrm{G}} \mathrm{H} 275 \mathrm{Y}$ mutant viruses exhibited the transmissibility equivalent to the wild-type virus in terms of efficiency and dynamics, which now can be correlated to the possession of all 4 permissive markers and the compensatory mutation in their NA proteins. In the NC99-like group, the severely attenuated transmissibility of GA/20/06 H275Y mutant virus now can be explained by the absence of entire 4 permissive markers in its NA protein. The MI/03/02 H275Y mutant virus also exhibited greatly attenuated transmissibility, which can be associated with the absence of the two permissive markers in its NA protein. This virus had acquired two permissive markers R222Q and D344N in its NA gene and its NA enzyme activity was the highest in the tested NC99-like NAs, so its attenuated transmissibility suggested that partial acquisition of the permissive markers was not sufficient to tolerate the negative effects of the $\mathrm{H} 275 \mathrm{Y}$ mutation, and all four permissive markers might be required for the full tolerance to the $\mathrm{H} 275 \mathrm{Y}$ mutation.

The contrasting difference in the transmissibility of two aberrant H1N1 viruses $\mathrm{NY} / 3467 / 09$ and MI/03/02 viruses also suggested that there might be a function hierarchy among the four NA permissive molecular markers in terms of their permissive degree to the H275Y mutation. The residues composition at the four positions in the NA genes of $\mathrm{NY} / 3467 / 09$ and $\mathrm{MI} / 03 / 02$ viruses is aberrant from the sequential addition order suggested by our analysis (V234M - R222Q - D344N - K329E). The transmissibility of the NY/3467/09 virus was essentially uncompromised only with early delay, in spite of the absence of the D344N substitution in its NA gene. However, the transmissibility of the $\mathrm{MI} / 03 / 02$ virus was still greatly attenuated in spite of the presence of the two permissive markers R222Q and D344N in its NA protein. The distinct transmissibility of the two viruses suggested that degree of the "permissiveness" of the four molecular markers to the H275Y mutation may be different, although each of them played a significant role in the NA protein function. It also suggested that all four of them were required to confer NA protein full tolerance to the $\mathrm{H} 275 \mathrm{Y}$ mutation to maintain unaltered virus fitness (transmissibility). By our results, although the D344N significantly increased the NA function, the D344N alone may only exert a small degree of "permissiveness" to the H275Y mutation as its absence in the NY/3467/09 virus seemed to have not attenuated the transmissibility of the viruses, and its presence in the MI/03/07 virus seemed to have not rescued the transmissibility of the virus. The functional hierarchy of the four permissive mutations doesn't necessarily correlate with the temporal sequence of the fixation of the four mutations, and further elucidations are warranted. 
With regards to the temporal sequential order for the four permissive molecular markers, we are uncertain of the underlying mechanism that prompted the first selection of the V234M during the NA divergence from NC99-like to BR07-like lineage. We showed that the V234M could increase the NA activity by increasing the NA protein expression, and all other three activity-increasing NA molecular markers appeared after the V234M. Apparently, the first appearance of V234M substitution made the NA protein promiscuous for more subsequent substitutions, especially the accumulation of activity-increasing NA substitutions. The underlying mechanism for such molecular selection is to be elucidated.

We also point out that the NA molecular basis most likely is not the only molecular basis in the naturally-occurring $\mathrm{H} 275 \mathrm{Y}$ resistant $\mathrm{H} 1 \mathrm{~N} 1$ viruses and we do not exclude the possible roles of other proteins in the emergence and spread of the naturally resistant H1N1 viruses. During 2008 2009, especially at the 2009 pre-pandemic season, the epidemiology data showed a trend that $\mathrm{H} 275 \mathrm{Y}$ mutant viruses were overriding the wild-type viruses and becoming the dominant circulating viruses(45;56), suggesting that the $\mathrm{H} 275 \mathrm{Y}$ mutant viruses had acquired better fitness including higher transmissibility than the wild-type viruses at the time. In the present study, we also noticed that both the $\mathrm{NY} / 1602 / 09$ and $\mathrm{rgBR} / 59 / 07-\mathrm{NA}^{\mathrm{H} 275 \mathrm{Y}+\mathrm{D} 354 \mathrm{G}} \mathrm{H} 275 \mathrm{Y}$ mutant virus possessed the four permissive markers and the compensatory mutation, but their transmissibility had subtle differences: the NY/1692/07 virus had apparently faster transmission dynamics than the wild-type virus, but the later had the same transmission as the wild-type virus. Therefore, although our findings of the NA molecular basis largely explain why the $\mathrm{rgBR} / 59 / 07-\mathrm{NA}^{\mathrm{H} 275 \mathrm{Y}+\mathrm{D} 354 \mathrm{G}}$ virus was not compromised compared to the wild-type viruses, it cannot explain why the NY/1692/09 virus exhibited even higher transmissibility than the wild-type virus, which is exactly in line with the trend of $\mathrm{H} 275 \mathrm{Y}$ mutant viruses in 2009 season. It suggests that the naturally resistant viruses could have acquired more advantageous fitness through other mechanisms potentially by other proteins, which made them even more "fit" than wild-type viruses and overriding in circulation. Other studies have shed some clues, such as some HA amino acid substitutions promoting the replication of the resistant viruses, and some intra-subtype inter-clade segment reassortment events associated with the naturally resistant viruses $(65 ; 66 ; 136)$. Further studies of the molecular basis other than NA in the naturally-occurring resistant viruses are warranted.

One important implication of this study is its relevance to the $\mathrm{H} 275 \mathrm{Y}$ mutation-caused resistance in the currently circulating swine-origin 2009 pandemic H1N1 (pH1N1) viruses. As for the virus fitness of H275Y mutant $\mathrm{pH} 1 \mathrm{~N} 1$ viruses, our study showed that a H275Y mutant $\mathrm{pH} 1 \mathrm{~N} 1$ viruses exhibited lower fitness than its counterpart wild-type $\mathrm{pH} 1 \mathrm{~N} 1$ virus including its less efficient transmissibility in ferrets and delayed growth in cells. In the NA molecular level, the attenuated virus fitness was well explained by significantly reduced NA enzyme activity due to greatly diminished protein expression and reduced enzyme affinity. The results strongly suggested that the NA of the pH1N1 virus had little tolerance to the H275Y mutation. However, we want to be very cautious to extend our findings and definition of the $\mathrm{H} 275 \mathrm{Y}$ tolerance to the pH1N1 NA. We have discovered the NA H275Y tolerance at the levels of NA enzyme 
activity, NA protein expression and NA enzyme affinity in H1N1 viruses of different lineages, but a perceptive standard needs to be further explored about how much degree of loss of activity, protein expression or enzyme affinity caused by the H275Y mutation can be defined as between tolerance and intolerance to the $\mathrm{H} 275 \mathrm{Y}$ mutation. In the case of the NA of the $\mathrm{H} 275 \mathrm{Y}$ mutant $\mathrm{pH} 1 \mathrm{~N} 1$ virus, the greatly reduced protein expression was rather conspicuous and could be easily defined as the intolerance to the $\mathrm{H} 275 \mathrm{Y}$ at the protein expression level, but the magnitude of decrease of the enzyme affinity was between those of the BR07-like viruses and the NC99-like viruses, thus the conclusion remained ambiguous about the intolerance or tolerance to the $\mathrm{H} 275 \mathrm{Y}$ at the enzyme affinity level. Moreover, with regards to the NA molecular basis for the tolerance to the H275Y mutation, we found the four permissive molecular markers (V234M, R222Q, D344N, K329E) and one compensatory mutation (D354G) in the NA genes seasonal H1N1 viruses. As seasonal N1 genes and 2009 pandemic N1 genes had distant ancestors (22), the questions remains uncertain if these markers play the same roles in NA proteins of $\mathrm{pH} 1 \mathrm{~N} 1$ viruses or if other amino acid residues can play similar roles. In the $\mathrm{pH} 1 \mathrm{~N} 1$ viruses, we have seen the difficulty to answer the questions, because the NAs showed diverse amino acid residues at some positions other than the ones we identified in seasonal NAs. How these variant amino acid residues can affect the NA protein expression or function warrants additional studies.

Our findings about NA tolerance to the $\mathrm{H} 275 \mathrm{Y}$ mutation also have implication on the surveillance monitoring of the NA inhibitor resistance, especially the $\mathrm{H} 275 \mathrm{Y}$ mutation-caused resistance. Our study underlines the necessity of not only continuous monitoring of drug resistance rates and but also characterization of evolving NA proteins. Our study showed that the H275Y mutant $\mathrm{pH} 1 \mathrm{~N} 1$ virus had lower fitness than the wild-type $\mathrm{pH} 1 \mathrm{~N} 1$ virus due to the NA intolerance to the $\mathrm{H} 275 \mathrm{Y}$ mutation at the protein expression level, which is still in line with the low the detection rate $(1 \sim 3.5 \%)$ of $\mathrm{H} 275 \mathrm{Y}$ mutant $\mathrm{pH} 1 \mathrm{~N} 1$ viruses in surveillance studies so far (73). However, some studies have found un-attenuated $\mathrm{H} 275 \mathrm{Y}$ mutant $\mathrm{pH} 1 \mathrm{~N} 1$ viruses in animal models $(87 ; 88)$, and several cases have identified community-transmitted $\mathrm{H} 275 \mathrm{Y}$ mutant $\mathrm{pH} 1 \mathrm{~N} 1$ viruses $(73 ; 75-78)$. In view of the precedent example of how the wild-type seasonal H1N1 viruses became the naturally-occurring oseltamivir-resistant $\mathrm{H} 1 \mathrm{~N} 1$ viruses by acquisition of only the four "permissive" molecular markers, it can be foreseen that the pH1N1 viruses also become the same naturally resistant through a similar NA evolution path. The question if $\mathrm{pH} 1 \mathrm{~N} 1$ viruses have started its path to become naturally resistant needs to be further evaluated. Ideally, we need closely monitor $\mathrm{pH} 1 \mathrm{~N} 1 \mathrm{NA}$ genotype divergence and investigate the corresponding changes in NA protein phenotypes. If the genotype of $\mathrm{pH} 1 \mathrm{~N} 1 \mathrm{NA}$ were found diverged, any big conspicuous changes associated with the genetic change including the NA activity, protein expression and enzyme affinity should raise an alarm about their possible acquisition of tolerance to the H275Y mutation. Further studies of these newly isolated $\mathrm{H} 275 \mathrm{Y}$ mutants of $\mathrm{H} 1 \mathrm{~N} 1 / 2009$ viruses are warranted, and the community-transmitted $\mathrm{H} 275 \mathrm{Y}$ mutant $\mathrm{pH} 1 \mathrm{~N} 1$ viruses will be of special merit for the investigation.

In summary, the present study uncovered how the NA proteins of the seasonal $\mathrm{H} 1 \mathrm{~N} 1$ viruses diverged during the evolution in terms of timeline, protein level attributes, 
and more importantly, the tolerance to the H275Y NA mutation. This study is of significance to understand the NA molecular basis for the emergence and spread of the naturally-occurring oseltamivir-resistant $\mathrm{H} 1 \mathrm{~N} 1$ viruses, and of important implication on the surveillance monitoring of the virus susceptibility to NA inhibitors. Current epidemic viruses continue evolving under selection pressure, therefore, such monitoring is essential for public health, as NA inhibitors remain the primary therapeutic option for influenza virus infection. 


\section{CHAPTER 4. METHODS}

\section{Compounds}

The NA inhibitors oseltamivir carboxylate ([3R,4R,5S]-4-acetamido-5-amino-3[1-ethylpropoxy]-1-cyclohexene-1-carboxylic acid) and zanamivir (4-guanidinoNeu5Ac2en) were provided by Hoffmann-La Roche, Ltd. (Basel, Switzerland). The compounds were dissolved in distilled water and aliquots were stored at $-20{ }^{\circ} \mathrm{C}$ until the time of use.

\section{Cells}

Madin-Darby canine kidney (MDCK) cells and human embryonic kidney cells transformed with large T antigen (293T cells) were obtained from American Type Culture Collection (ATCC, Manassas, VA). MDCK cells were maintained in minimal essential medium (MEM) supplemented with $5 \%$ fetal bovine serum (FBS), $5 \mathrm{mM}$ L-glutamine, $0.2 \%$ sodium bicarbonate, $100 \mathrm{U} / \mathrm{ml}$ penicillin, $100 \mu \mathrm{g} / \mathrm{ml}$ streptomycin sulfate, and $100 \mu \mathrm{g} / \mathrm{ml}$ kanamycin sulfate. 293T cells were maintained in opti-MEM (Invitogen) supplemented with 5\% FBS and antibiotics as above. MDCK cells transfected with cDNA encoding human 2,6-sialyltransferase (MDCK-SIAT1 cells) were kindly provided by Dr Mikhail N. Matrosovich and maintained as described previously. Baby Hamster Kidney (BHK) cells were kindly provided by Dr. Charles Russell and maintained as described previously (137). The Normal Human Bronchial Epithelial (NHBE) cells (Lonza, Basel, Switzerland) were cultured on an air-liquid interface in 12 -well plates and the infection with influenza viruses was described as previously (138). For growth curve, the inoculums were added to the apical surface of cells and were removed after $1 \mathrm{~h}$ of incubation at $37^{\circ} \mathrm{C}$. Apical supernatants containing progeny viruses were collected by adding fresh medium into apical compartment 30 mins prior to the indicated time point and stored at $-70^{\circ} \mathrm{C}$ for further titration in MDCK cells All the cell lines were maintained in a humidified atmosphere of $5 \% \mathrm{CO}_{2}$ at $37{ }^{\circ} \mathrm{C}$.

\section{Viruses}

A panel of pandemic H1N1 and seasonal H1N1 influenza viruses of which the NA genes were previously sequenced and published on Genebank was chosen for the studies (Table 2-1 and Table 3-2). Among them, pandemic A/Denmark/524/2009 virus $(\mathrm{DM} / 524 / 09)$ and A/ Denmark/528/2009 virus (DM/528/09) were provided by Statens

Serum Institute, Copenhagen, Denmark. A/New York/3467/2009 virus (NY/3467/09) and A/New York/1692/2009 virus (NY/1692/09) were provided by Wadsworth center of the New York state department of health. Wild-type or NA H275Y mutant of A/Mississipii/03/2002 virus (MI/03/02) was provided by the Neuraminidase Inhibitor Susceptibility Network. All the rest of viruses such as A/Brisbane/59/2007 virus (A/BR/59/09), A/Hawii/28/2008 virus (HW/28/08), A/New Jersey/15/2007 virus 
(A/NJ/15/07), A/Georgia/17/2006 virus (GA/17/06), A/Georgia/20/2006 virus (GA/20/06), A/Memphis/13/2006 virus (MEM/13/06) etc viruses were provided by U.S Centers for Disease Control and Prevention. Stocks of the H1N1 viruses were prepared in MDCK cells. Upon receiving the viruses, all strains of virus underwent a limited number of (1 2) passages in MDCK cells to maintain their original properties.

\section{Virus Sequence Analysis}

Viral RNA was isolated by using the RNeasy Mini kit (Qiagen, Valencia, CA). Samples were reverse-transcribed and analyzed by PCR using primers specific for the NA gene segment, as described previously (139). Sequencing was performed by the Hartwell Center for Bioinformatics and Biotechnology at St. Jude Children's Research Hospital. The DNA template was sequenced by using rhodamine or dRhodamine dye terminator cycle-sequencing Ready Reaction kits with AmpliTaq DNA polymerase FS (Perkin-Elmer, Applied Biosystems, Inc., Foster City, CA) and synthetic oligonucleotides. Samples were analyzed in a Perkin-Elmer Applied Biosystems DNA sequencer (model 373 or 377). DNA sequences were completed and edited by using the Lasergene sequence analysis software package (DNASTAR, Madison, WI).

\section{Plasmid Construction and Site-directed Mutagenesis}

The full length cDNA of 8 segments of BR/59/07 virus, and NA segments of $\mathrm{GA} / 17 / 06$ and GA/20/06 viruses were cloned into the $\mathrm{pHW} 2000$ plasmid vector for generation of reverse-genetics viruses in cells, as described previously (140). The respective mutations indicated in the results were introduced into the corresponding NA genes by using QuickChange site-directed mutagenesis (Stratagene, La Jolla, CA) and confirmed by Sanger sequencing. Selected NA genes listed in the results were also coned into the pCAGGS plasmid vector for expression of recombinant proteins in cells, as described previously (137). The HA epitop-tag sequence were added into the primer sequences to express the epitop-tagged NA protein by the vector.

\section{Generation of Reverse-genetics Influenza Viruses}

Reverse-genetics viruses were rescued by transfecting the PHW2000 plasmids containing 8 influenza segments into 293T/MDCK cells co-culture, as describe previously (141). Variant NA segments listed in the results were used together other 7 segments from BR/59/07 virus respectively for rescuing the viruses. One passage of the rescued supernatant in MDCK cells (at MOI of 0.001 0.01) were used to prepare stocks of all reverse-genetics viruses. 


\section{Generation and Detection of Recombinant NA Proteins}

Recombinant NA proteins were generated by transfecting the respective pCAGSS plasmids into BHK cells, as described previously (137), and the protein expression was detected by western blot or flowcytometry. Generally, for the proteins which needed comparison among them, the same amount of each pCAGGS plasmid was transfected into respective wells of BHK cells in parallel. Then the cell pellet was harvested at same time in parallel, usually 24 hour post transfection for further analysis. For following analysis, the same amount of cells were used and assayed in parallel. Anti-HA epitope monoclonal antibodies (Sigma) were used for the detection of the recombinant proteins in flowcytometry or webstern blot, as described previously (142). Anti-Actin monoclonal antibodies were used for detection of $\beta$-Actin (Sigma) in the cells for normalization purpose. The pCAGGS plasmids of different NA gene variants are as indicated in the results.

\section{Determination of Infectivity of Influenza Viruses}

The $50 \%$ tissue culture infectious dose $\left(\mathrm{TCID}_{50}\right)$ was determined in MDCK cells. The cells were infected with serial log dilutions of the stock viruses, incubated for $1 \mathrm{~h}$ at $37^{\circ} \mathrm{C}$, washed, and overlaid with infection medium (MEM with $0.3 \%$ BSA and $1 \mu \mathrm{g} / \mathrm{ml}$ TPCK-trypsin). Infection of cells was determined by hemagglutination assay (HI) after incubation for $3 \mathrm{~d}$ at $37^{\circ} \mathrm{C}$, and $\mathrm{TCID}_{50}$ was calculated by the Reed-Muench method. The plaque assay was used to determine the plaque forming unit ( $\mathrm{pfu}$ ) in a virus stock. Confluent MDCK cells were incubated for $1 \mathrm{~h}$ at $37^{\circ} \mathrm{C}$ with 10 -fold serial dilutions of virus in $1 \mathrm{ml}$ of infection medium. The cells were then washed and overlaid with freshly prepared MEM containing $0.3 \%$ BSA, $0.9 \%$ bacto-agar, and $1 \mu \mathrm{g} / \mathrm{ml}$ TPCK trypsin. The plaques were visualized after incubation at $37^{\circ} \mathrm{C}$ for $3 \mathrm{~d}$ by staining with $0.1 \%$ crystal violet solution containing $10 \%$ formaldehyde.

\section{Replication Kinetics}

Single-step or multi-step growth curves were generated for influenza viruses in MDCK cells. For single-step growth curve, confluent cell monolayers were infected with viruses at a multiplicity of infection (MOI) of $\sim 2.0 \mathrm{PFU} /$ cell. After incubation, the cells were washed with $0.9 \%$ aqueous $\mathrm{NaCl}$ solution $(\mathrm{pH} 2.2)$ to remove free infectious virus particles and then were washed twice with phosphate-buffered saline (PBS) to adjust the $\mathrm{pH}$. Supernatants were collected 2, 4, 6, 8, 10 and $12 \mathrm{~h}$ p.i. and stored at $-70^{\circ} \mathrm{C}$ for titration. To generate multi-step growth curves, MDCK cells were infected with viruses at an MOI of $0.001 \mathrm{PFU} /$ cell. Supernatants were collected 12, 24, 36, 48, 60 and $72 \mathrm{~h}$ p.i. and stored at $-70^{\circ} \mathrm{C}$ for titration. 


\section{Virus Susceptibility to NA Inhibitors}

The viral NA activity was determined first by a modified fluorometric assay using the fluorogenic substrate 2'-(4-methylumbelliferyl)- $\alpha$-D-N-acetylneuraminic acid (MUNANA) (Sigma-Aldrich). The fluorescence of the released 4-methylumbelliferone was measured in a Synergy 2 multi-mode microplate reader (BioTek) using excitation and emission wavelengths of 360 and $460 \mathrm{~nm}$, respectively. The drug concentration required to inhibit $50 \%$ of the NA enzymatic activity $\left(\mathrm{IC}_{50}\right)$ was determined by plotting the percent inhibition of NA activity as a function of compound concentration calculated in the GraphPad Prism 4 software from the inhibitor-response curve. The NA inhibitor-sensitive A/Fukui/20/04 (H3N2) influenza virus was included in every plate for comparison.

\section{NA Enzyme Kinetics}

All H1N1 viruses were standardized to an equivalent dose of $10^{6.0} \mathrm{PFU} / \mathrm{ml}$. We measured NA enzyme kinetics at pH 6.5 with 33 mM 2-(N-Morpholino) ethanesulfonic acid hydrate (MES; Sigma-Aldrich), $4 \mathrm{mM} \mathrm{CaCl}_{2}$, and MUNANA with a final substrate concentration of 0 to $800 \mu \mathrm{M}$. The reaction was conducted at $37^{\circ} \mathrm{C}$ in a total volume of $50 \mu \mathrm{l}$, and the fluorescence of released 4-methylumbelliferone was measured every $60 \mathrm{sec}$ for $60 \mathrm{~min}$ in a Synergy 2 multi-mode microplate reader (BioTek) using excitation and emission wavelengths of 360 and $460 \mathrm{~nm}$, respectively. The $\mathrm{Km}$ and Vmax were calculated by fitting the data to the appropriate Michaelis-Menten equations by using nonlinear regression in the GraphPad Prism 4 software.

\section{Virus Transmission Experiments in Ferrets}

The animal work with the pandemic H1N1/2009 influenza viruses was performed in an animal biosafety level 3+(ABSL3+) laboratory approved by the U.S. Department of Agriculture. The animal work with seasonal H1N1 influenza viruses was conducted in ABSL2+ laboratory under applicable laws and guidelines and after approval from the St. Jude Children's Research Hospital Animal Care and Use Committee. The ferrets for the pandemic H1N1 virus study were were obtained from the ferret breeding program at St. Jude Children's Research Hospital. The ferrets for the seasonal H1N1 virus study were obtained from Triple F farm. All ferrets were 4-5 months of age and were sero-negative for contemporary influenza A H1N1 and H3N2 viruses and for influenza B viruses. Ferrets were firstly housed in the isolators in and monitored for 3-5 days to establish baseline body temperature and overall health. Donor ferrets were initially housed separately from contact ferrets.

The experiment settings were slightly different between the studies for pandemic and seasonal H1N1 viruses. For the study of pandemic viruses, one donor ferrets were lightly anesthetized with isoflurane and inoculated with $10^{6}$ TCID $_{50}$ of each of the pandemic $\mathrm{H} 1 \mathrm{~N} 1$ viruses in $1.0 \mathrm{ml}$ sterile PBS respectively. One donor ferret was 
inoculated with $10^{6} \mathrm{TCID}_{50}$ of a mixture of $\mathrm{A} / \mathrm{DM} / 524 / 09$ and $\mathrm{A} / \mathrm{DM} / 528 / 09$ viruses $(1: 1$ infectivity ratio). After the donor ferrets were confirmed to shed virus on day 2 p.i. by the Directigen Flu A+B quick test (BD, Franklin Lakes, NJ), each was then housed with 2 naïve direct-contact ferrets in the same cage. Two additional recipient ferrets were placed in an adjacent cage isolated from the donor's cage by a two layers of wire mesh $(\sim 5 \mathrm{~cm}$ apart) that prevented physical contact but allowed the passage of respiratory droplets. Borazine gun (Zero Toys, Concord, MA ) tested that the airflow was non-directional inside the isolator. Thus, the transmission setting was 1 donor +2 direct-contact +2 respiratory-droplet recipient ferrets for each tested pandemic virus.

For the study of seasonal viruses, three donor ferrets were inoculated with $10^{5}$ TCID $_{50}$ each of the seasonal H1N1 viruses in $1.0 \mathrm{ml}$ sterile PBS respectively as the results listed. Then at day $1 \mathrm{p} . \mathrm{i}$, each was then housed with 1 naïve direct-contact ferrets in the same cages. One additional naïve ferret was placed in an adjacent cage separated by double layers of perforated dividers ( $\sim 5 \mathrm{~cm}$ apart $)$ for the purpose of respiratory-droplet transmission. The cages were oriented to direct the airflow from the donor cage side to the respiratory-droplet recipient cage side. Thus the transmission setting was 3 replicates of 1 donor +1 direct-contact +1 respiratory-droplet recipient ferret for each virus. For both studies, Ferret nasal wash samples were collected at day 1 p.i and the following every other day from day 2 p.i for 14 days. Ferret weight, temperature and other clinical signs were recorded daily for 21 days. Body temperature was measured by subcutaneous implantable temperature transponders (Bio Medic Data Systems Inc, Seaford, DE).

\section{Collection and Titration of Nasal Wash Samples}

Nasal washes were collected from donor and recipient ferrets on day 1, 2, 4, 6, 8, 10,12 , and 14 p.i. by flushing ferret both nostrils with $1.0 \mathrm{ml} \mathrm{PBS}$, and TCID $_{50}$ titers were determined in MDCK cells. Inflammatory cell counts were determined for the pandemic virus study. Briefly, the nasal washes were centrifuged at $2000 \mathrm{rpm}$ for $5 \mathrm{~min}$. The pellet was resuspended in PBS, and the total cell number was counted in a hemacytometer under light microscopy. Inflammation was defined as a cell count $\geq 10$ times the baseline count which was determined before the inoculation or exposure.

\section{Serologic Tests}

Serum samples were collected from ferrets 3 weeks after virus inoculation, treated with receptor-destroying enzyme, heat-inactivated at $56^{\circ} \mathrm{C}$ for $30 \mathrm{~min}$, and tested by $\mathrm{HI}$ assay with $0.5 \%$ packed Turkey red blood cells (TRBC).

\section{Quantitative Single Nucleotide Polymorphism Assay}

The quantitative single nucleotide polymorphism (SNP) assay was performed to determine the proportion of wild-type or $\mathrm{H} 275 \mathrm{Y}$ mutant viruses in mixed sample for the 
pandemic virus study as described previously (138), with slight modification. Briefly, an NA fragment (nucleotide 673 to 1034) containing the codon at NA 275 position was amplified by RT-PCR. Single nucleotide primer extension was performed using a SNaPshot kit (ABI) per the manufacturer's protocol. A extension probe was used to detect the first nucleotide of NA 275 codon. After the SNaPshot reaction, the product was analyzed by ABI 3730xl capillary electrophoresis instrument (ABI) per the manufacturer's protocol. Data were analyzed by using ABI GeneMapper software.

\section{Statistical Analysis}

The unpaired $t$-test or analysis of variance (ANOVA) was used for all

comparisons, if the significance was indicated in the result description, then $\mathrm{p}<0.05$. 


\section{LIST OF REFERENCES}

1. Cheung,T.K., and Poon,L.L. 2007. Biology of influenza a virus. Ann. N. Y. Acad. Sci. 1102:1-25.

2. Salomon,R., and Webster,R.G. 2009. The influenza virus enigma. Cell 136:402-410.

3. Guan,Y., Shortridge,K.F., Krauss,S., and Webster,R.G. 1999. Molecular characterization of $\mathrm{H} 9 \mathrm{~N} 2$ influenza viruses: were they the donors of the "internal" genes of H5N1 viruses in Hong Kong? Proc. Natl. Acad. Sci. U. S. A 96:9363-9367.

4. $\quad$ Fouchier,R.A., Schneeberger,P.M., Rozendaal,F.W., Broekman,J.M., Kemink,S.A., Munster,V., Kuiken,T., Rimmelzwaan,G.F., Schutten,M., Van Doornum,G.J. et al 2004. Avian influenza A virus (H7N7) associated with human conjunctivitis and a fatal case of acute respiratory distress syndrome. Proc. Natl. Acad. Sci. U. S. A 101:1356-1361.

5. Claas,E.C., Osterhaus,A.D., van Beek R., de Jong,J.C., Rimmelzwaan,G.F., Senne,D.A., Krauss,S., Shortridge,K.F., and Webster,R.G. 1998. Human influenza A H5N1 virus related to a highly pathogenic avian influenza virus. Lancet 351:472-477.

6. Brownstein,J.S., Freifeld,C.C., and Madoff,L.C. 2009. Influenza A (H1N1) virus, 2009--online monitoring. N. Engl. J. Med. 360:2156.

7. Dawood,F.S., Jain,S., Finelli,L., Shaw,M.W., Lindstrom,S., Garten,R.J., Gubareva,L.V., Xu,X., Bridges,C.B., and Uyeki,T.M. 2009. Emergence of a novel swine-origin influenza A (H1N1) virus in humans. N. Engl. J. Med. 360:2605-2615.

8. Yuen,K.Y., Chan,P.K., Peiris,M., Tsang,D.N., Que,T.L., Shortridge,K.F., Cheung,P.T., To,W.K., Ho,E.T., Sung,R. et al 1998. Clinical features and rapid viral diagnosis of human disease associated with avian influenza A H5N1 virus. Lancet 351:467-471.

9. WHO 2009. Cumulative number of confirmed human cases of avian influenza $\mathrm{A} /(\mathrm{H} 5 \mathrm{~N} 1)$ reported to $\mathrm{WHO}$.

10. Peter Palese, and Megan L.Shaw 2007. Orthomyxoviridae: The Viruses and Their Replication. In Fields Virology. D.M.Knipe, and Howley,P.M., editors. Lippincott Williams \& Wilkins. 1648-1688.

11. Chen,W., Calvo,P.A., Malide,D., Gibbs,J., Schubert,U., Bacik,I., Basta,S., O'Neill,R., Schickli,J., Palese,P. et al 2001. A novel influenza A virus mitochondrial protein that induces cell death. Nat. Med. 7:1306-1312. 
12. Neumann,G., and Kawaoka,Y. 2006. Host range restriction and pathogenicity in the context of influenza pandemic. Emerg. Infect. Dis. 12:881-886.

13. Neumann,G., Noda,T., and Kawaoka,Y. 2009. Emergence and pandemic potential of swine-origin H1N1 influenza virus. Nature 459:931-939.

14. Rogers,G.N., and Paulson,J.C. 1983. Receptor determinants of human and animal influenza virus isolates: differences in receptor specificity of the $\mathrm{H} 3$ hemagglutinin based on species of origin. Virology 127:361-373.

15. Kawaoka,Y., and Webster,R.G. 1988. Sequence requirements for cleavage activation of influenza virus hemagglutinin expressed in mammalian cells. Proc. Natl. Acad. Sci. U. S. A 85:324-328.

16. Subbarao,E.K., London,W., and Murphy,B.R. 1993. A single amino acid in the PB2 gene of influenza A virus is a determinant of host range. J. Virol. 67:1761-1764.

17. Hatta,M., Gao,P., Halfmann,P., and Kawaoka,Y. 2001. Molecular basis for high virulence of Hong Kong H5N1 influenza A viruses. Science 293:1840-1842.

18. Steel,J., Lowen,A.C., Mubareka,S., and Palese,P. 2009. Transmission of influenza virus in a mammalian host is increased by PB2 amino acids $627 \mathrm{~K}$ or 627E/701N. PLoS. Pathog. 5:e1000252.

19. Mazur,I., Anhlan,D., Mitzner,D., Wixler,L., Schubert,U., and Ludwig,S. 2008. The proapoptotic influenza A virus protein $\mathrm{PB} 1-\mathrm{F} 2$ regulates viral polymerase activity by interaction with the PB1 protein. Cell Microbiol. 10:1140-1152.

20. Conenello,G.M., Zamarin,D., Perrone,L.A., Tumpey,T., and Palese,P. 2007. A single mutation in the PB1-F2 of H5N1 (HK/97) and 1918 influenza A viruses contributes to increased virulence. PLoS. Pathog. 3:1414-1421.

21. Garcia-Sastre,A. 2001. Inhibition of interferon-mediated antiviral responses by influenza A viruses and other negative-strand RNA viruses. Virology 279:375384.

22. Neumann,G., Noda,T., and Kawaoka,Y. 2009. Emergence and pandemic potential of swine-origin H1N1 influenza virus. Nature 459:931-939.

23. Jiao,P., Tian,G., Li,Y., Deng,G., Jiang,Y., Liu,C., Liu,W., Bu,Z., Kawaoka,Y., and Chen,H. 2008. A single-amino-acid substitution in the NS1 protein changes the pathogenicity of $\mathrm{H} 5 \mathrm{~N} 1$ avian influenza viruses in mice. $J$. Virol. 82:11461154.

24. Seo,S.H., Hoffmann,E., and Webster,R.G. 2002. Lethal H5N1 influenza viruses escape host anti-viral cytokine responses. Nat. Med. 8:950-954. 
25. Roberts NA, and Govorkova EA 2009. The Activity of Neuraminidase Inhibitor Oseltamivir Against All Subtypes of Influenza Viruses. In Global View of the Fight against Influenza. Mitrasinovic PM, editor. Nova Science Publishers. 93118.

26. Wagner,R., Matrosovich,M., and Klenk,H.D. 2002. Functional balance between haemagglutinin and neuraminidase in influenza virus infections. Rev. Med. Virol. 12:159-166.

27. Hensley,S.E., Das,S.R., Gibbs,J.S., Bailey,A.L., Schmidt,L.M., Bennink,J.R., and Yewdell,J.W. 2011. Influenza A virus hemagglutinin antibody escape promotes neuraminidase antigenic variation and drug resistance. PLoS. One. 6:e15190.

28. Govorkova,E.A., and McCullers,J.A. 2012. Therapeutics Against Influenza. Curr. Top. Microbiol. Immunol.

29. Weinstock,D.M., and Zuccotti,G. 2006. Adamantane resistance in influenza A. JAMA 295:934-936.

30. Hayden,F.G., and Pavia,A.T. 2006. Antiviral management of seasonal and pandemic influenza. J. Infect. Dis. 194 Suppl 2:S119-S126.

31. Hayden,F.G., and de,J. 2011. Emerging influenza antiviral resistance threats. $J$. Infect. Dis. 203:6-10.

32. Lackenby,A., Thompson,C.I., and Democratis,J. 2008. The potential impact of neuraminidase inhibitor resistant influenza. Curr. Opin. Infect. Dis. 21:626-638.

33. Birnkrant,D., and Cox,E. 2009. The Emergency Use Authorization of peramivir for treatment of 2009 H1N1 influenza. N. Engl. J. Med. 361:2204-2207.

34. Gubareva,L.V., Kaiser,L., and Hayden,F.G. 2000. Influenza virus neuraminidase inhibitors. Lancet 355:827-835.

35. Colman,P.M., Hoyne,P.A., and Lawrence,M.C. 1993. Sequence and structure alignment of paramyxovirus hemagglutinin-neuraminidase with influenza virus neuraminidase. J. Virol. 67:2972-2980.

36. von Itzstein,M., Wu,W.Y., Kok,G.B., Pegg,M.S., Dyason,J.C., Jin,B., Van,P.T., Smythe,M.L., White,H.F., Oliver,S.W. et al 1993. Rational design of potent sialidase-based inhibitors of influenza virus replication. Nature 363:418-423.

37. Varghese,J.N., Colman,P.M., van Donkelaar,A., Blick,T.J., Sahasrabudhe,A., and Kimm-Breschkin,J.L. 1997. Structural evidence for a second sialic acid binding site in avian influenza virus neuraminidases. Proc. Natl. Acad. Sci. U. S. A 94:11808-11812. 
38. Okomo-Adhiambo,M., Sleeman,K., Ballenger,K., Nguyen,H.T., Mishin,V.P., Sheu,T.G., Smagala,J., Li,Y., Klimov,A.I., and Gubareva,L.V. 2010.

Neuraminidase inhibitor susceptibility testing in human influenza viruses: a laboratory surveillance perspective. Viruses. 2:2269-2289.

39. Domingo,E., Menendez-Arias,L., and Holland,J.J. 1997. RNA virus fitness. Rev. Med. Virol. 7:87-96.

40. McCaw,J.M., Arinaminpathy,N., Hurt,A.C., McVernon,J., and McLean,A.R. 2011. A mathematical framework for estimating pathogen transmission fitness and inoculum size using data from a competitive mixtures animal model. PLoS. Comput. Biol. 7:e1002026.

41. Kiso,M., Mitamura,K., Sakai-Tagawa,Y., Shiraishi,K., Kawakami,C., Kimura,K., Hayden,F.G., Sugaya,N., and Kawaoka,Y. 2004. Resistant influenza A viruses in children treated with oseltamivir: descriptive study. Lancet 364:759-765.

42. Schirmer,P., and Holodniy,M. 2009. Oseltamivir for treatment and prophylaxis of influenza infection. Expert. Opin. Drug Saf 8:357-371.

43. Das,K., Ma,L.C., Xiao,R., Radvansky,B., Aramini,J., Zhao,L., Marklund,J., Kuo,R.L., Twu,K.Y., Arnold,E. et al 2008. Structural basis for suppression of a host antiviral response by influenza A virus. Proc. Natl. Acad. Sci. U. S. A 105:13093-13098.

44. Das,K., Aramini,J.M., Ma,L.C., Krug,R.M., and Arnold,E. 2010. Structures of influenza A proteins and insights into antiviral drug targets. Nat. Struct. Mol. Biol. 17:530-538.

45. Moscona,A. 2009. Global transmission of oseltamivir-resistant influenza. $N$. Engl. J. Med. 360:953-956.

46. Monto,A.S., Kimm-Breschkin,J.L., Macken,C., Hampson,A.W., Hay,A., Klimov,A., Tashiro,M., Webster,R.G., Aymard,M., Hayden,F.G. et al 2006. Detection of influenza viruses resistant to neuraminidase inhibitors in global surveillance during the first 3 years of their use. Antimicrob. Agents Chemother. 50:2395-2402.

47. Stephenson,I., Democratis,J., Lackenby,A., McNally,T., Smith,J., Pareek,M., Ellis,J., Bermingham,A., Nicholson,K., and Zambon,M. 2009. Neuraminidase inhibitor resistance after oseltamivir treatment of acute influenza A and B in children. Clin. Infect. Dis. 48:389-396.

48. Mungall,B.A., Xu,X., and Klimov,A. 2004. Surveillance of influenza isolates for susceptibility to neuraminidase inhibitors during the 2000-2002 influenza seasons. Virus Res. 103:195-197. 
49. Escuret,V., Frobert,E., Bouscambert-Duchamp,M., Sabatier,M., Grog,I., Valette,M., Lina,B., Morfin,F., and Ferraris,O. 2008. Detection of human influenza $\mathrm{A}(\mathrm{H} 1 \mathrm{~N} 1)$ and B strains with reduced sensitivity to neuraminidase inhibitors. J. Clin. Virol. 41:25-28.

50. Sheu,T.G., Deyde,V.M., Okomo-Adhiambo,M., Garten,R.J., Xu,X., Bright,R.A., Butler,E.N., Wallis,T.R., Klimov,A.I., and Gubareva,L.V. 2008. Surveillance for neuraminidase inhibitor resistance among human influenza A and B viruses circulating worldwide from 2004 to 2008. Antimicrob. Agents Chemother. 52:3284-3292.

51. Bouvier,N.M., Lowen,A.C., and Palese,P. 2008. Oseltamivir-resistant influenza A viruses are transmitted efficiently among guinea pigs by direct contact but not by aerosol. J. Virol. 82:10052-10058.

52. Herlocher,M.L., Carr,J., Ives,J., Elias,S., Truscon,R., Roberts,N., and Monto,A.S. 2002. Influenza virus carrying an R292K mutation in the neuraminidase gene is not transmitted in ferrets. Antiviral Res. 54:99-111.

53. Herlocher,M.L., Truscon,R., Elias,S., Yen,H.L., Roberts,N.A., Ohmit,S.E., and Monto,A.S. 2004. Influenza viruses resistant to the antiviral drug oseltamivir: transmission studies in ferrets. J. Infect. Dis. 190:1627-1630.

54. Ives,J.A., Carr,J.A., Mendel,D.B., Tai,C.Y., Lambkin,R., Kelly,L., Oxford,J.S., Hayden,F.G., and Roberts,N.A. 2002. The H274Y mutation in the influenza A/H1N1 neuraminidase active site following oseltamivir phosphate treatment leave virus severely compromised both in vitro and in vivo. Antiviral Res. 55:307-317.

55. Carr,J., Ives,J., Kelly,L., Lambkin,R., Oxford,J., Mendel,D., Tai,L., and Roberts,N. 2002. Influenza virus carrying neuraminidase with reduced sensitivity to oseltamivir carboxylate has altered properties in vitro and is compromised for infectivity and replicative ability in vivo. Antiviral Res. 54:7988 .

56. Weinstock,D.M., and Zuccotti,G. 2009. The evolution of influenza resistance and treatment. JAMA 301:1066-1069.

57. Meijer,A., Lackenby,A., Hungnes,O., Lina,B., van-der-Werf,S., Schweiger,B., Opp,M., Paget,J., van-de-Kassteele,J., Hay,A. et al 2009. Oseltamivir-resistant influenza virus A (H1N1), Europe, 2007-08 season. Emerg. Infect. Dis. 15:552560 .

58. Hauge,S.H., Dudman,S., Borgen,K., Lackenby,A., and Hungnes,O. 2009. Oseltamivir-resistant influenza viruses A (H1N1), Norway, 2007-08. Emerg. Infect. Dis. 15:155-162. 
59. Hurt,A.C., Ernest,J., Deng,Y.M., Iannello,P., Besselaar,T.G., Birch,C., Buchy,P., Chittaganpitch,M., Chiu,S.C., Dwyer,D. et al 2009. Emergence and spread of oseltamivir-resistant A(H1N1) influenza viruses in Oceania, South East Asia and South Africa. Antiviral Res. 83:90-93.

60. Dharan,N.J., Gubareva,L.V., Meyer,J.J., Okomo-Adhiambo,M., McClinton,R.C., Marshall,S.A., St,G.K., Epperson,S., Brammer,L., Klimov,A.I. et al 2009. Infections with oseltamivir-resistant influenza $A(H 1 N 1)$ virus in the United States. JAMA 301:1034-1041.

61. Baz,M., Abed,Y., Simon,P., Hamelin,M.E., and Boivin,G. 2010. Effect of the neuraminidase mutation $\mathrm{H} 274 \mathrm{Y}$ conferring resistance to oseltamivir on the replicative capacity and virulence of old and recent human influenza A(H1N1) viruses. J. Infect. Dis. 201:740-745.

62. Agrawal,A.S., Sarkar,M., Ghosh,S., Roy,T., Chakrabarti,S., Lal,R., Mishra,A.C., Chadha,M.S., and Chawla-Sarkar,M. 2010. Genetic characterization of circulating seasonal Influenza A viruses (2005-2009) revealed introduction of oseltamivir resistant H1N1 strains during 2009 in eastern India. Infect. Genet. Evol. 10:1188-1198.

63. Casalegno,J.S., Bouscambert-Duchamp,M., Caro,V., Schuffenecker,I., Sabatier,M., Traversier,A., Valette,M., Lina,B., Ferraris,O., and Escuret,V. 2010. Oseltamivir-resistant influenza A(H1N1) viruses in south of France, 2007/2009. Antiviral Res. 87:242-248.

64. Eshaghi,A., Bolotin,S., Burton,L., Low,D.E., Mazzulli,T., and Drews,S.J. 2009. Genetic microheterogeneity of emerging H275Y influenza virus A (H1N1) in Toronto, Ontario, Canada from the 2007-2008 respiratory season. J. Clin. Virol. 45:142-145.

65. Yang,J.R., Lin,Y.C., Huang,Y.P., Su,C.H., Lo,J., Ho,Y.L., Yao,C.Y., Hsu,L.C., Wu,H.S., and Liu,M.T. 2011. Reassortment and mutations associated with emergence and spread of oseltamivir-resistant seasonal influenza A/H1N1 viruses in 2005-2009. PLoS. One. 6:e18177.

66. Zaraket,H., Saito,R., Suzuki,Y., Baranovich,T., Dapat,C., Caperig-Dapat,I., and Suzuki,H. 2010. Genetic makeup of amantadine-resistant and oseltamivirresistant human influenza A/H1N1 viruses. J. Clin. Microbiol. 48:1085-1092.

67. Bloom,J.D., Gong,L.I., and Baltimore,D. 2010. Permissive secondary mutations enable the evolution of influenza oseltamivir resistance. Science 328:12721275 .

68. Rameix-Welti,M.A., Enouf,V., Cuvelier,F., Jeannin,P., and van der,W.S. 2008. Enzymatic properties of the neuraminidase of seasonal H1N1 influenza viruses provide insights for the emergence of natural resistance to oseltamivir. PLoS. Pathog. 4:e1000103. 
69. Collins,P.J., Haire,L.F., Lin,Y.P., Liu,J., Russell,R.J., Walker,P.A., Martin,S.R., Daniels,R.S., Gregory,V., Skehel,J.J. et al 2009. Structural basis for oseltamivir resistance of influenza viruses. Vaccine 27:6317-6323.

70. Rameix-Welti,M.A., Munier,S., Le,G.S., Cuvelier,F., Agou,F., Enouf,V., Naffakh,N., and van der,W.S. 2011. Neuraminidase of 2007-2008 influenza A(H1N1) viruses shows increased affinity for sialic acids due to the D344N substitution. Antivir. Ther. 16:597-603.

71. Abed,Y., Pizzorno,A., Bouhy,X., and Boivin,G. 2011. Role of permissive neuraminidase mutations in influenza A/Brisbane/59/2007-like (H1N1) viruses. PLoS. Pathog. 7:e1002431.

72. 2009. Update: drug susceptibility of swine-origin influenza A (H1N1) viruses, April 2009. MMWR Morb. Mortal. Wkly. Rep. 58:433-435.

73. Hurt,A.C., Chotpitayasunondh,T., Cox,N.J., Daniels,R., Fry,A.M., Gubareva,L.V., Hayden,F.G., Hui,D.S., Hungnes,O., Lackenby,A. et al 2012. Antiviral resistance during the 2009 influenza A H1N1 pandemic: public health, laboratory, and clinical perspectives. Lancet Infect. Dis. 12:240-248.

74. European Centre for Disease Prevention and Control 2009. ECDC THREAT ASSESSMENT: First isolation of a secondary oseltamivir-resistant A(H1N1)v strain in Denmark. 1-3.

75. WHO 2009. Oseltamivir resistance in immunocompromised hospital patients.

76. 2009. Oseltamivir-resistant novel influenza A (H1N1) virus infection in two immunosuppressed patients - Seattle, Washington, 2009. MMWR Morb. Mortal. Wkly. Rep. 58:893-896.

77. 2009. Oseltamivir-resistant 2009 pandemic influenza A (H1N1) virus infection in two summer campers receiving prophylaxis--North Carolina, 2009. MMWR Morb. Mortal. Wkly. Rep. 58:969-972.

78. Hurt,A.C., Hardie,K., Wilson,N.J., Deng,Y.M., Osbourn,M., Gehrig,N., and Kelso,A. 2011. Community transmission of oseltamivir-resistant A(H1N1)pdm09 influenza. N. Engl. J. Med. 365:2541-2542.

79. Hurt,A.C., Deng,Y.M., Ernest,J., Caldwell,N., Leang,L., Iannello,P., Komadina,N., Shaw,R., Smith,D., Dwyer,D.E. et al 2011. Oseltamivir-resistant influenza viruses circulating during the first year of the influenza $\mathrm{A}(\mathrm{H} 1 \mathrm{~N} 1)$ 2009 pandemic in the Asia-Pacific region, March 2009 to March 2010. Euro. Surveill 16. 
80. Gubareva,L.V., Trujillo,A.A., Okomo-Adhiambo,M., Mishin,V.P., Deyde,V.M., Sleeman,K., Nguyen,H.T., Sheu,T.G., Garten,R.J., Shaw,M.W. et al 2010. Comprehensive assessment of 2009 pandemic influenza A (H1N1) virus drug susceptibility in vitro. Antivir. Ther. 15:1151-1159.

81. Hurt,A.C., Lee,R.T., Leang,S.K., Cui,L., Deng,Y.M., Phuah,S.P., Caldwell,N., Freeman,K., Komadina,N., Smith,D. et al 2011. Increased detection in Australia and Singapore of a novel influenza A(H1N1)2009 variant with reduced oseltamivir and zanamivir sensitivity due to a S247N neuraminidase mutation. Euro. Surveill 16.

82. Nguyen,H.T., Fry,A.M., Loveless,P.A., Klimov,A.I., and Gubareva,L.V. 2010. Recovery of a multidrug-resistant strain of pandemic influenza A 2009 (H1N1) virus carrying a dual $\mathrm{H} 275 \mathrm{Y} / \mathrm{I} 223 \mathrm{R}$ mutation from a child after prolonged treatment with oseltamivir. Clin. Infect. Dis. 51:983-984.

83. Pizzorno,A., Bouhy,X., Abed,Y., and Boivin,G. 2011. Generation and characterization of recombinant pandemic influenza $\mathrm{A}(\mathrm{H} 1 \mathrm{~N} 1)$ viruses resistant to neuraminidase inhibitors. J. Infect. Dis. 203:25-31.

84. Duan,S., Boltz,D.A., Seiler,P., Li,J., Bragstad,K., Nielsen,L.P., Webby,R.J., Webster,R.G., and Govorkova,E.A. 2010. Oseltamivir-resistant pandemic H1N1/2009 influenza virus possesses lower transmissibility and fitness in ferrets. PLoS. Pathog. 6:e1001022.

85. Hamelin,M.E., Baz,M., Abed,Y., Couture,C., Joubert,P., Beaulieu,E., Bellerose,N., Plante,M., Mallett,C., Schumer,G. et al 2010. Oseltamivirresistant pandemic $\mathrm{A} / \mathrm{H} 1 \mathrm{~N} 1$ virus is as virulent as its wild-type counterpart in mice and ferrets. PLoS. Pathog. 6:e1001015.

86. Hamelin,M.E., Baz,M., Bouhy,X., Beaulieu,E., Dube,K., Mallett,C., and Boivin,G. 2011. Reduced airborne transmission of oseltamivir-resistant pandemic A/H1N1 virus in ferrets. Antivir. Ther. 16:775-779.

87. Kiso,M., Shinya,K., Shimojima,M., Takano,R., Takahashi,K., Katsura,H., Kakugawa,S., Le,M.T., Yamashita,M., Furuta,Y. et al 2010. Characterization of oseltamivir-resistant $2009 \mathrm{H} 1 \mathrm{~N} 1$ pandemic influenza A viruses. PLoS. Pathog. 6.

88. Seibert,C.W., Kaminski,M., Philipp,J., Rubbenstroth,D., Albrecht,R.A., Schwalm,F., Stertz,S., Medina,R.A., Kochs,G., Garcia-Sastre,A. et al 2010. Oseltamivir-resistant variants of the 2009 pandemic H1N1 influenza A virus are not attenuated in the guinea pig and ferret transmission models. J. Virol. 84:11219-11226. 
89. Van der Vries E., Veldhuis Kroeze,E.J., Stittelaar,K.J., Linster,M., Van der,L.A., Schrauwen,E.J., Leijten,L.M., van,A.G., Schutten,M., Kuiken,T. et al 2011. Multidrug resistant $2009 \mathrm{~A} / \mathrm{H} 1 \mathrm{N1}$ influenza clinical isolate with a neuraminidase I223R mutation retains its virulence and transmissibility in ferrets. PLoS. Pathog. 7:e1002276.

90. Pizzorno,A., Abed,Y., Bouhy,X., Beaulieu,E., Mallett,C., Russell,R., and Boivin,G. 2012. Impact of mutations at residue i223 of the neuraminidase protein on the resistance profile, replication level, and virulence of the 2009 pandemic influenza virus. Antimicrob. Agents Chemother. 56:1208-1214.

91. Seibert,C.W., Rahmat,S., Krammer,F., Palese,P., and Bouvier,N.M. 2012. Efficient transmission of pandemic H1N1 influenza viruses with high-level oseltamivir resistance. J. Virol.

92. de Jong,J.C., Claas,E.C., Osterhaus,A.D., Webster,R.G., and Lim,W.L. 1997. A pandemic warning? Nature 389:554.

93. Subbarao,K., Klimov,A., Katz,J., Regnery,H., Lim,W., Hall,H., Perdue,M., Swayne,D., Bender,C., Huang,J. et al 1998. Characterization of an avian influenza A (H5N1) virus isolated from a child with a fatal respiratory illness. Science 279:393-396.

94. Smith,J.R., Rayner,C.R., Donner,B., Wollenhaupt,M., Klumpp,K., and Dutkowski,R. 2011. Oseltamivir in seasonal, pandemic, and avian influenza: a comprehensive review of 10-years clinical experience. Adv. Ther. 28:927-959.

95. Earhart,K.C., Elsayed,N.M., Saad,M.D., Gubareva,L.V., Nayel,A., Deyde,V.M., Abdelsattar,A., Abdelghani,A.S., Boynton,B.R., Mansour,M.M. et al 2009. Oseltamivir resistance mutation N294S in human influenza A(H5N1) virus in Egypt. J. Infect. Public Health 2:74-80.

96. de Jong,M.D., Tran,T.T., Truong,H.K., Vo,M.H., Smith,G.J., Nguyen,V.C., Bach,V.C., Phan,T.Q., Do,Q.H., Guan,Y. et al 2005. Oseltamivir resistance during treatment of influenza A (H5N1) infection. N. Engl. J. Med. 353:26672672.

97. Le,Q.M., Kiso,M., Someya,K., Sakai,Y.T., Nguyen,T.H., Nguyen,K.H., Pham,N.D., Ngyen,H.H., Yamada,S., Muramoto,Y. et al 2005. Avian flu: isolation of drug-resistant H5N1 virus. Nature 437:1108.

98. Govorkova,E.A., Ilyushina,N.A., Boltz,D.A., Douglas,A., Yilmaz,N., and Webster,R.G. 2007. Efficacy of oseltamivir therapy in ferrets inoculated with different clades of H5N1 influenza virus. Antimicrob. Agents Chemother. 51:1414-1424. 
99. Yen,H.L., Ilyushina,N.A., Salomon,R., Hoffmann,E., Webster,R.G., and Govorkova,E.A. 2007. Neuraminidase inhibitor-resistant recombinant A/Vietnam/1203/04 (H5N1) influenza viruses retain their replication efficiency and pathogenicity in vitro and in vivo. J. Virol. 81:12418-12426.

100. Kiso,M., Ozawa,M., Le,M.T., Imai,H., Takahashi,K., Kakugawa,S., Noda,T., Horimoto,T., and Kawaoka,Y. 2011. Effect of an asparagine-to-serine mutation at position 294 in neuraminidase on the pathogenicity of highly pathogenic H5N1 influenza A virus. J. Virol. 85:4667-4672.

101. Govorkova,E.A., Ilyushina,N.A., Marathe,B.M., McClaren,J.L., and Webster,R.G. 2010. Competitive fitness of oseltamivir-sensitive and -resistant highly pathogenic H5N1 influenza viruses in a ferret model. J. Virol. 84:80428050 .

102. Bright,R.A., Shay,D.K., Shu,B., Cox,N.J., and Klimov,A.I. 2006. Adamantane resistance among influenza A viruses isolated early during the 2005-2006 influenza season in the United States. JAMA 295:891-894.

103. Deyde,V.M., Xu,X., Bright,R.A., Shaw,M., Smith,C.B., Zhang,Y., Shu,Y., Gubareva,L.V., Cox,N.J., and Klimov,A.I. 2007. Surveillance of resistance to adamantanes among influenza $\mathrm{A}(\mathrm{H} 3 \mathrm{~N} 2)$ and $\mathrm{A}(\mathrm{H} 1 \mathrm{~N} 1)$ viruses isolated worldwide. J. Infect. Dis. 196:249-257.

104. Bright,R.A., Shay,D.K., Shu,B., Cox,N.J., and Klimov,A.I. 2006. Adamantane resistance among influenza A viruses isolated early during the 2005-2006 influenza season in the United States. JAMA 295:891-894.

105. WHO 2010. WHO Guidelines for Pharmacological Management of Pandemic (H1N1) 2009 Influenza and other Influenza Viruses.

106. Abed,Y., Baz,M., and Boivin,G. 2006. Impact of neuraminidase mutations conferring influenza resistance to neuraminidase inhibitors in the N1 and N2 genetic backgrounds. Antivir. Ther. 11:971-976.

107. Kimm-Breschkin,J., Trivedi,T., Hampson,A., Hay,A., Klimov,A., Tashiro,M., Hayden,F., and Zambon,M. 2003. Neuraminidase sequence analysis and susceptibilities of influenza virus clinical isolates to zanamivir and oseltamivir. Antimicrob. Agents Chemother. 47:2264-2272.

108. Ward,P., Small,I., Smith,J., Suter,P., and Dutkowski,R. 2005. Oseltamivir (Tamiflu) and its potential for use in the event of an influenza pandemic. $J$ Antimicrob. Chemother. 55 Suppl 1:i5-i21.

109. Moscona,A. 2009. Global transmission of oseltamivir-resistant influenza. $N$. Engl. J. Med. 360:953-956. 
110. Baz,M., Abed,Y., Papenburg,J., Bouhy,X., Hamelin,M.E., and Boivin,G. 2009. Emergence of oseltamivir-resistant pandemic $\mathrm{H} 1 \mathrm{~N} 1$ virus during prophylaxis. $N$ Engl J Med 361:2296-2297.

111. Leung,T.W., Tai,A.L., Cheng,P.K., Kong,M.S., and Lim,W. 2009. Detection of an oseltamivir-resistant pandemic influenza $\mathrm{A} / \mathrm{H} 1 \mathrm{~N} 1$ virus in Hong Kong. $J$. Clin. Virol. 46:298-299.

112. Zonis,Z., Engelhard,D., Hindiyeh,M., Ram,D., Mandelboim,M., Mendelson,E., and Glikman,D. 2010. Community-acquired Oseltamivir-Resistant Pandemic (H1N1) 2009 in Child, Israel. Emerg. Infect. Dis. 16:1045-1046.

113. WHO 2009. Oseltamivir resistance in immunocompromised hospital patients.

114. Gulland,A. 2009. First cases of spread of oseltamivir resistant swine flu between patients are reported in Wales. BMJ 339:b4975.

115. Yen,H.L., Herlocher,L.M., Hoffmann,E., Matrosovich,M.N., Monto,A.S., Webster,R.G., and Govorkova,E.A. 2005. Neuraminidase inhibitor-resistant influenza viruses may differ substantially in fitness and transmissibility. Antimicrob. Agents Chemother. 49:4075-4084.

116. Yen,H.L., Herlocher,L.M., Hoffmann,E., Matrosovich,M.N., Monto,A.S., Webster,R.G., and Govorkova,E.A. 2005. Neuraminidase inhibitor-resistant influenza viruses may differ substantially in fitness and transmissibility. Antimicrob. Agents Chemother. 49:4075-4084.

117. Matrosovich,M., Matrosovich,T., Carr,J., Roberts,N.A., and Klenk,H.D. 2003. Overexpression of the alpha-2,6-sialyltransferase in MDCK cells increases influenza virus sensitivity to neuraminidase inhibitors. J Virol. 77:8418-8425.

118. Matrosovich,M., Matrosovich,T., Carr,J., Roberts,N.A., and Klenk,H.D. 2003. Overexpression of the alpha-2,6-sialyltransferase in MDCK cells increases influenza virus sensitivity to neuraminidase inhibitors. $J$ Virol. 77:8418-8425.

119. Carr,J., Ives,J., Kelly,L., Lambkin,R., Oxford,J., Mendel,D., Tai,L., and Roberts,N. 2002. Influenza virus carrying neuraminidase with reduced sensitivity to oseltamivir carboxylate has altered properties in vitro and is compromised for infectivity and replicative ability in vivo. Antiviral Res. 54:7988 .

120. Tellier,R. 2009. Aerosol transmission of influenza A virus: a review of new studies. J. R. Soc. Interface 6 Suppl 6:S783-S790.

121. Tellier,R. 2006. Review of aerosol transmission of influenza A virus. Emerg. Infect. Dis. 12:1657-1662. 
122. Kohlmeier,J.E., and Woodland,D.L. 2009. Immunity to respiratory viruses. Anпu. Rev. Immunol. 27:61-82.

123. Mathews,J.D., Chesson,J.M., McCaw,J.M., and McVernon,J. 2009. Understanding influenza transmission, immunity and pandemic threats. Influenza. Other Respi. Viruses. 3:143-149.

124. Kohlmeier,J.E., and Woodland,D.L. 2009. Immunity to respiratory viruses. Annu. Rev. Immunol. 27:61-82.

125. Gubareva,L.V., and Fry,A.M. 2010. Current challenges in the risk assessment of neuraminidase inhibitor-resistant influenza viruses. $J$ Infect. Dis. 201:656-658.

126. Ibe,S., Fujisaki,S., Fujisaki,S., Morishita,T., and Kaneda,T. 2006. Quantitative SNP-detection method for estimating HIV-1 replicative fitness: application to protease inhibitor-resistant viruses. Microbiol. Immunol. 50:765-772.

127. Govorkova,E.A., and McCullers,J.A. 2012. Therapeutics Against Influenza. Curr. Top. Microbiol. Immunol.

128. Chao,D.L., Bloom,J.D., Kochin,B.F., Antia,R., and Longini,I.M., Jr. 2012. The global spread of drug-resistant influenza. J. R. Soc. Interface 9:648-656.

129. Holmes,E.C. 2010. Virology. Helping the resistance. Science 328:1243-1244.

130. Air,G.M., Laver,W.G., Webster,R.G., Els,M.C., and Luo,M. 1989. Antibody recognition of the influenza virus neuraminidase. Cold Spring Harb. Symp. Quant. Biol. 54 Pt 1:247-255.

131. Gulati,U., Hwang,C.C., Venkatramani,L., Gulati,S., Stray,S.J., Lee,J.T., Laver,W.G., Bochkarev,A., Zlotnick,A., and Air,G.M. 2002. Antibody epitopes on the neuraminidase of a recent $\mathrm{H} 3 \mathrm{~N} 2$ influenza virus (A/Memphis/31/98). $J$. Virol. 76:12274-12280.

132. Lentz,M.R., Air,G.M., Laver,W.G., and Webster,R.G. 1984. Sequence of the neuraminidase gene of influenza virus $\mathrm{A} / \mathrm{Tokyo} / 3 / 67$ and previously uncharacterized monoclonal variants. Virology 135:257-265.

133. Sandbulte,M.R., Westgeest,K.B., Gao,J., Xu,X., Klimov,A.I., Russell,C.A., Burke,D.F., Smith,D.J., Fouchier,R.A., and Eichelberger,M.C. 2011. Discordant antigenic drift of neuraminidase and hemagglutinin in H1N1 and H3N2 influenza viruses. Proc. Natl. Acad. Sci. U. S. A 108:20748-20753.

134. Webster,R.G., Laver,W.G., Air,G.M., and Schild,G.C. 1982. Molecular mechanisms of variation in influenza viruses. Nature 296:115-121. 
135. Bloom,J.D., Nayak,J.S., and Baltimore,D. 2011. A computational-experimental approach identifies mutations that enhance surface expression of an oseltamivirresistant influenza neuraminidase. PLoS. One. 6:e22201.

136. Ginting,T.E., Shinya,K., Kyan,Y., Makino,A., Matsumoto,N., Kaneda,S., and Kawaoka,Y. 2012. Amino acid changes in hemagglutinin contribute to the replication of oseltamivir-resistant H1N1 influenza viruses. J. Virol. 86:121127.

137. Reed,M.L., Bridges,O.A., Seiler,P., Kim,J.K., Yen,H.L., Salomon,R., Govorkova,E.A., Webster,R.G., and Russell,C.J. 2010. The pH of activation of the hemagglutinin protein regulates $\mathrm{H} 5 \mathrm{~N} 1$ influenza virus pathogenicity and transmissibility in ducks. J. Virol. 84:1527-1535.

138. Duan,S., Boltz,D.A., Li,J., Oshansky,C.M., Marjuki,H., Barman,S., Webby,R.J., Webster,R.G., and Govorkova,E.A. 2011. Novel genotyping and quantitative analysis of neuraminidase inhibitor resistance-associated mutations in influenza a viruses by single-nucleotide polymorphism analysis. Antimicrob. Agents Chemother. 55:4718-4727.

139. Hoffmann,E., Stech,J., Guan,Y., Webster,R.G., and Perez,D.R. 2001. Universal primer set for the full-length amplification of all influenza A viruses. Arch. Virol. 146:2275-2289.

140. Hoffmann,E., Neumann,G., Kawaoka,Y., Hobom,G., and Webster,R.G. 2000. A DNA transfection system for generation of influenza A virus from eight plasmids. Proc. Natl. Acad. Sci. U. S. A 97:6108-6113.

141. Hoffmann,E., Mahmood,K., Yang,C.F., Webster,R.G., Greenberg,H.B., and Kemble,G. 2002. Rescue of influenza B virus from eight plasmids. Proc. Natl. Acad. Sci. U. S. A 99:11411-11416.

142. Marjuki,H., Yen,H.L., Franks,J., Webster,R.G., Pleschka,S., and Hoffmann,E. 2007. Higher polymerase activity of a human influenza virus enhances activation of the hemagglutinin-induced Raf/MEK/ERK signal cascade. Virol. J. $4: 134$. 


\section{APPENDIX. NOVEL GENOTYPING AND QUANTITATIVE ANALYSIS OF NEURAMINIDASE INHIBITOR RESISTANCE-ASSOCIATED MUTATIONS IN INFLUENZA A VIRUSES BY SINGLE-NUCLEOTIDE POLYMORPHISM ANALYSIS}

Duan,S., Boltz,D.A., Li,J., Oshansky,C.M., Marjuki,H., Barman,S., Webby,R.J., Webster,R.G., and Govorkova,E.A. 2011. Novel genotyping and quantitative analysis of neuraminidase inhibitor resistance-associated mutations in influenza a viruses by single-nucleotide polymorphism analysis. Antimicrob. Agents Chemother. 55:4718-4727.

Follow this link: http://aac.asm.org/content/55/10/4718.long 


\section{VITA}

Susu Duan was born in Suzhou, Anhui Province, China in 1980. She graduated from Suzhou No.1 high school in June 1998. Following graduation, she entered into Anhui Medical University and she obtained bachelor degree in medicine majoring in preventative medicine in June 2003. Following graduation, she entered into Peking Medical College and Chinese Academy of Medical Science and she obtained master degree in science majoring in pathogenic microbiology at June 2006. She was enrolled into the Interdisciplinary Program of Biomedical Sciences at the University Tennessee Health Science Center in August 2007 and conducted her dissertation research with a focus on influenza virus virology in Dr. Robert G. Webster's lab at St. Jude Children's Research Hospital. She is expected to obtain her PhD degree in May 2012. 